\title{
THE EFFECT OF ARTERY BIFURCATION ANGLES ON FLUID FLOW AND WALL SHEAR STRESS IN THE MIDDLE CEREBRAL ARTERY
}

\author{
A Thesis \\ Presented to \\ the Faculty of California Polytechnic State University, \\ San Luis Obispo
}

\author{
In Partial Fulfillment \\ of the Requirements for the Degree \\ Master of Science in Biomedical Engineering
}

by

Zachary Ramey Jones

December 2014 
(C)2014

Zachary Ramey Jones

ALL RIGHTSRESERVED 


\section{COMMITTEEMEMBERSHIP}

TITLE:

AUTHOR:

DATE SUBMITTED:

COMMITTE CHAIR:

COMMITTEE MEMBER:

COMMITTEE MEMBER:
The Effect of Artery Bifurcation Angles on Fluid Flow and Wall Shear Stress in the Middle Cerebral Artery

Zachary Ramey Jones

December 2014

David Clague, Ph.D., Associate Professor, Department of Biomedical \& General Engineering

Scott Hazelwood, Ph.D., Associate Professor, Department of Biomedical \& General Engineering

Dan Walsh, Ph.D., Associate Professor, Department of Biomedical \& General Engineering 


\author{
ABSTRACT \\ The Effect of Artery Bifurcation Angles on Fluid Flow and Wall \\ Shear Stress in the Middle Cerebral Artery \\ Zachary Ramey Jones
}

Saccular aneurysms are the abnormal plastic deformation of veins and arteries that can lead to lethal thrombus genesis or internal hemorrhaging. Medication and surgery greatly reduce the mortality rates, but treatment is limited by predicting who will develop aneurysms. A common location for saccular aneurysm genesis is at the main middle cerebral artery (MCA) bifurcation. The main MCA bifurcation is comprised of the M1 MCA segment, parent artery, and two M2 segments, daughter arteries. Studies have found that the lateral angle (LA) ratio of the MCA bifurcation is correlated with aneurysm formation. The LA ratio is defined as the angle between the M1 and the larger M2 divided by the angle between the M1 and the smaller M2. When the LA ratio is equal to 1, perfectly symmetrical, no aneurysms are found at the MCA bifurcation. When the LA ratio is greater than 1.6, aneurysms are commonly found at the MCA bifurcation. In the research described here, varying MCA bifurcation angles were compared to uncover any changes to fluid flow and wall shear stress that could stimulate aneurysm growth. Eight pre-aneurysm MCA bifurcation models were created in SolidWorks ${ }^{\circledR}$ using 120 degrees, 90 degrees, and 60 degrees as the angle between the M1 and the larger M2. LA ratios of 1, 1.6 and 2.2 were then used to characterize the other branch angle (60 degrees with a LA ratio of 1 was excluded). These models were imported into COMSOL Multiphysics® where the laminar fluid flow module was used to simulate non-Newtonian blood flow. Fluid flow profiles showed little to no change between the models. Shear stress changed when the LA ratio was increased, but the changed varied between the 120, 90 and 60 degree models. 120 degree models had a 3.87\% decrease in max shear stress with a LA ratio of 2.2 while the 90 degree models had $7.5 \%$ decrease in max shear stress with a LA ratio of 2.2. Each daughter artery had distinct areas of high shear stress when the LA ratio equaled 1. Increasing the LA ratio or decreasing the bifurcation angle caused the areas of shear stress to merge together. Increasing LA ratio caused shear stress to decrease and spread around the MCA bifurcation. The reduction in max wall shear stress for high LA ratios supports current aneurysm genesis hypothesizes, but additional testing is required before bifurcation geometries can be used to predicted aneurysm genesis. 


\section{TABLE OF CONTENTS}

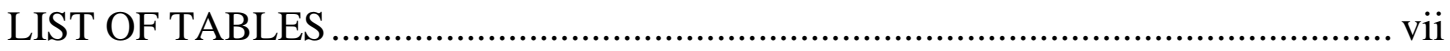

LIST OF FIGURES …………………........................................................... vii

CHAPTER 1: INTRODUCTION ………………………..................................

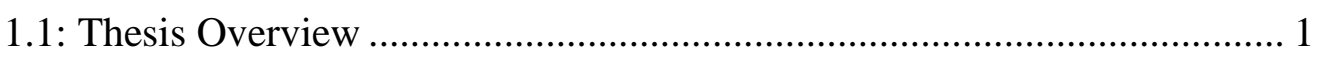

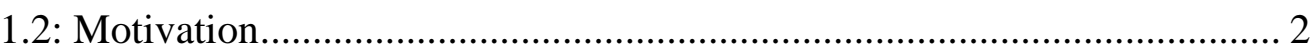

1.3: Previous Aneurysm Studies ................................................................. 3

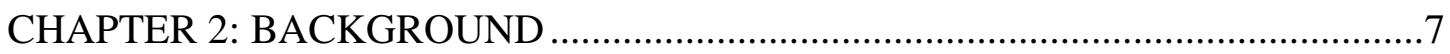

2.1: Artery Physiology …………………………………............................. 7

2.2: Aneurysm Physiology and Pathology ……………….............................. 9

2.3: Middle Cerebral Artery Bifurcation Characterization .............................. 14

2.4: Loading Conditions.............................................................................. 20

2.5: Rheology and Shear Stress .................................................................. 29

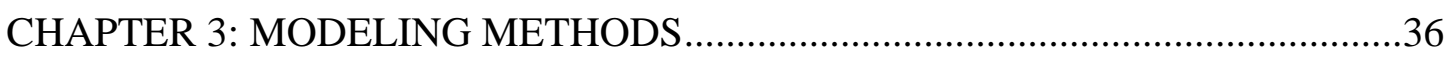

3.1: Modeling Overview ............................................................................... 36

3.2: Fluid Flow Module and Loading Conditions............................................ 37

3.3: Geometries of Model ......................................................................... 40

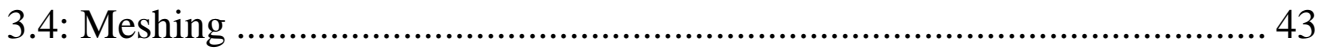

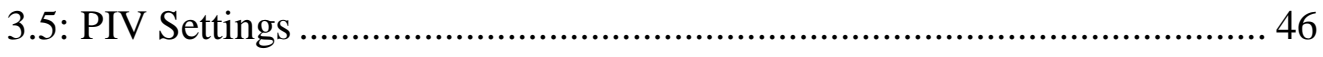

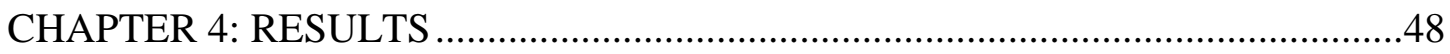

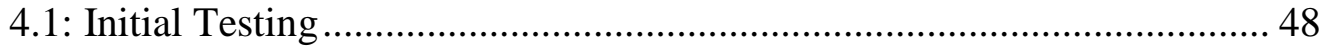

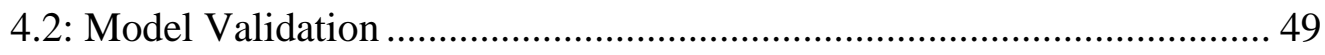

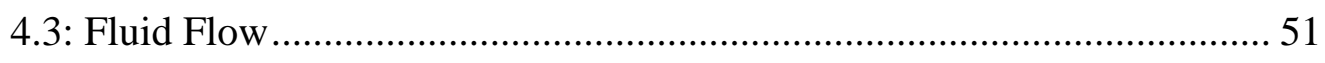

4.4: Wall Shear Stress ................................................................................. 53

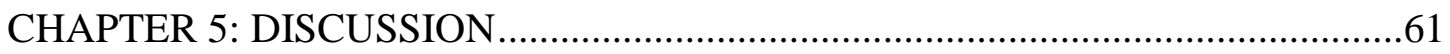

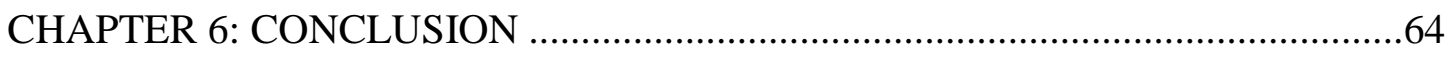

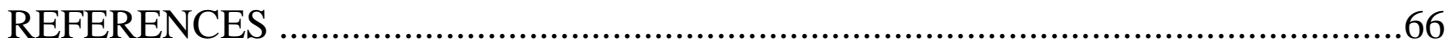

APPENDICES

APPENDIX A: GEOMETRIES FOR SOLIDWORKS MODELS ..................70

APPENDIX B: FLOW PROFILE AND SHEAR STRESS PLOTS ................74 
APPENDIX C: MATLAB CODE FOR WINDKESSEL MODEL................82

APPENDIX D: COMSOL PIV INSTRUCTIONS ...................................85

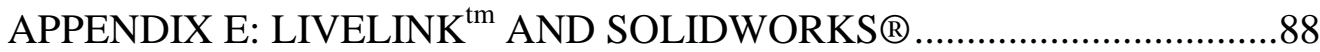




\section{LIST OF TABLES}

Table

Page

I. Convergence study of fluid velocities and shear stress for 120-120 model at 0.3 second

II. Comparison between analytical equations and simulation results for simple cylinder.

III. Comparison of literature values to MCA control model ...................................49

IV. Table of averaged inlet and outlet flow rates for all models...............................53

V. Max shear stress during systole and diastole with maximum area of shear

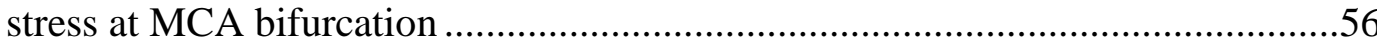

VI. Percent change in shear stress between different models compared to 120-120 control model 


\section{LIST OF FIGURES}

Figure

Page

1. FEA of wall shear stress and blood velocity in a MCA bifurcation with an aneurysm [9].

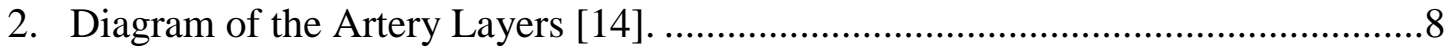

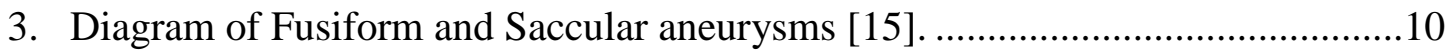

4. Example of surgical clipping of an aneurysm at a bifurcation [20].....................12

5. Diagram of Cerebral Arteries [18]. The area of interest is the main MCA bifurcation located in the middle left side of the diagram. ................................16

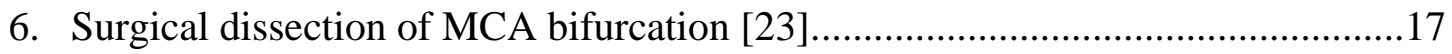

7. Representation of healthy LA and DA ratios [1]. .(a)The entire cerebral vasculature. (b and c)Lateral angle measurements.

8. Representation of a MCA bifurcation with an aneurysm [1].(a)The entire cerebral vasculature. (b and c)Lateral angle measurements.

9. Two, Three, and Four Element Windkessel Model Diagram [33].

10. Graph comparing the 3-element Windkessel model to measured pressure in a human aorta [35].

11. Example of a 3-element Windkessel model of Howe's original code for the

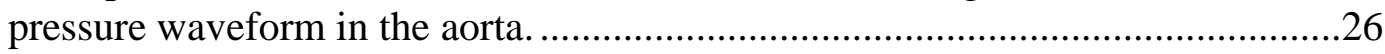

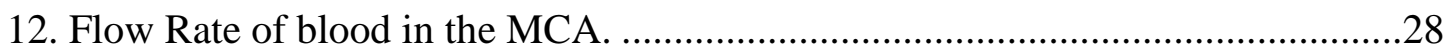

13. Simulated Windkessel cardiac pressure waveform for the MCA........................29

14. Hypothesized pathways for mechanical stimuli to ecNOS activation [42] ..........33

15. Velocity profile of Idealized 2D bifurcation with non-Newtonian laminar flow ..37

16. SolidWorks model of an idealized healthy MCA bifurcation. .............................41

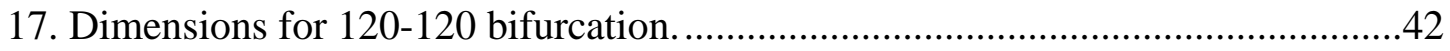

18. Line plot of probed shear stress at the bifurcation related to Degrees of freedom.

19. Mesh of the boundary and domain elements for $120^{\circ}-120^{\circ}$ model. 
20. (left)PIV experiment from literature. (right)Particle tracings from simulated model.

21. Flow profile at peak systole for all bifurcation angles. (a-h) $120^{\circ}-120^{\circ}, 120^{\circ}-$ $75^{\circ}, 120^{\circ}-54.54^{\circ}, 90^{\circ}-90^{\circ}, 90^{\circ}-56.25^{\circ}, 90^{\circ}-40.91^{\circ}, 60^{\circ}-37.5^{\circ}, 60^{\circ}-27-27^{\circ}$ respectively.

22. Shear Stress Comparison. (a-h) $120^{\circ}-120^{\circ}, 120^{\circ}-75^{\circ}, 120^{\circ}-54.54^{\circ}, 90^{\circ}-90^{\circ}$, $90^{\circ}-56.25^{\circ}, 90^{\circ}-40.91^{\circ}, 60^{\circ}-37.5^{\circ}, 60^{\circ}-27-27^{\circ}$ respectively.

23. Shear stress versus Distance from bifurcation for $120^{\circ}$ base angle. .57

24. Shear stress versus Distance from bifurcation for $90^{\circ}$ base angle.......................58

25. Shear stress versus Distance from bifurcation for $60^{\circ}$ base angle.......................58

26. Probed max shear stress of all models over one cardiac cycle ..........................60

27. Dimensions of 120 degree base angle model with LA ratio $1.6\left(120^{\circ}-75^{\circ}\right) \ldots \ldots \ldots . .70$

28. Dimensions of 120 degree base angle model with LA ratio $2.2\left(120^{\circ}-54.54^{\circ}\right) \ldots . .70$

29. Dimensions of 90 degree base angle model with LA ratio $1\left(90^{\circ}-90^{\circ}\right) \ldots \ldots \ldots \ldots \ldots . . . . .71$

30. Dimensions of 90 degree base angle model with LA ratio $1.6\left(90^{\circ}-56.25^{\circ}\right) \ldots \ldots . . .71$

31. Dimensions of 90 degree base angle model with LA ratio $2.2\left(90^{\circ}-40.91^{\circ}\right) \ldots \ldots . . .72$

32. Dimensions of 60 degree base angle model with LA ratio $1.6\left(60^{\circ}-37.5^{\circ}\right) \ldots \ldots \ldots . . .72$

33. Dimensions of 60 degree base angle model with LA ratio $2.2\left(60^{\circ}-27.27^{\circ}\right) \ldots \ldots . . .73$

34. (top)Velocity plot of 120 degree base angle model with LA ratio 1.0.

(bottom)Shear stress plot of 120 degree base angle model with LA ratio 1.0.......74

35. (top)Velocity plot of 120 degree base angle model with LA ratio 1.6. (bottom)Shear stress plot of 120 degree base angle model with LA ratio 1.6.......75

36. (top)Velocity plot of 120 degree base angle model with LA ratio 2.2. (bottom)Shear stress plot of 120 degree base angle model with LA ratio 2.2.......76

37. (top)Velocity plot of 90 degree base angle model with LA ratio 1.0. (bottom)Shear stress plot of 90 degree base angle model with LA ratio 1.0.........77

38. (top)Velocity plot of 90 degree base angle model with LA ratio 1.6. (bottom)Shear stress plot of 90 degree base angle model with LA ratio 1.6.........78

39. (top)Velocity plot of 90 degree base angle model with LA ratio 2.2. (bottom)Shear stress plot of 90 degree base angle model with LA ratio 2.2 ........79 
40. (top)Velocity plot of 60 degree base angle model with LA ratio 1.6. (bottom)Shear stress plot of 60 degree base angle model with LA ratio 1.6........80

41. (top)Velocity plot of 60 degree base angle model with LA ratio 2.2. (bottom)Shear stress plot of 60 degree base angle model with LA ratio 2.2 ........81 


\section{CHAPTER 1: INTRODUCTION}

\section{1: Thesis Overview}

Currently, there are no reliable ways to predict who will develop an aneurysm. Multiple studies have been performed to determine the etiology of aneurysms, but all of them have been inconclusive. This thesis will expand upon the study performed by Takashi Sadatomo, et al., [1] in 2013 where they correlated the occurrence of aneurysms in the main middle cerebral artery (MCA) to asymmetry between the bifurcation angles. The study characterized the bifurcation angle using the lateral angle (LA) ratio, defined as the angle of the M1 MCA segment to the larger M2 segment divided by the angle of the M1 to the other, smaller M2 segment. LA ratio of 1.0 indicated perfect symmetry while greater LA ratios indicated increased asymmetry. The study found that LA ratios greater than 1.6 in the MCA bifurcation were associated with the presence of an aneurysm. The goal of this thesis was to create MCA models using the morphological differences found in the study by Sadatomo to examine changes in the fluid flow and wall shear stress and how they pertain to aneurysm genesis. Additionally, this thesis will lay the foundation for future aneurysm models for Cal Poly Biofluidics Laboratory.

This thesis hypothesizes that lateral angle ratios greater than 1.6 at the MCA bifurcation cause alterations in fluid flow and wall shear stress. Background research of the MCA regarding morphology, physiology, loading conditions, properties of blood, flow rates, and previous aneurysm studies was performed. To test the effect of bifurcation angles, COMSOL Multiphysics® was used to perform finite element analysis (FEA) using the laminar flow module to simulate blood flow through the main MCA bifurcation. One control model was created, with an additional seven other models with 
varying bifurcation angles. All models were simulated over two cardiac cycles. To verify the simulations, the control MCA model was compared to literature values and a particle image velocimetry (PIV) experiment. The flow rates, wall shear stress during systole and diastole, area affected by high shear stress, and shear stress over the cardiac cycle were compared between models. Current aneurysm etiology hypothesis were then compared to the results to better understand aneurysm genesis in the MCA with regards to bifurcation symmetry.

\section{2: Motivation}

Cerebrovascular diseases are the fourth leading cause of death in America behind heart disease, cancer, and lower respiratory diseases. In 2011 alone, cerebrovascular diseases killed 128,931 people [2]. Included in this category are cerebral aneurysms. Cerebral aneurysms are the abnormal expansion of cerebral arteries and veins leading to intracranial bleeding and thrombus genesis. Cerebral aneurysms rupture at a rate of 10 out of 100,000 people per year [3]. This translate to about 30,000 cases a year in the United States [3]. Ruptured aneurysms primarily occur between the ages of 30-70 years old, with a peak around 60-70 years old [4]. Ruptured aneurysms are the main cause for spontaneous subarachnoid hemorrhaging (SAHa). SAHa has a high mortality rate with $10 \%$ of cases dying on route to the hospital and an additional $8 \%$ dying from excessive bleeding during surgery [4]. Of the survivors, two thirds report permanent reduction in quality of life [4].

The chance of developing a cerebral aneurysm in a life time is $2 \%$, with $30 \%$ of cases having multiple aneurysms present, but the rate varies between countries [4]. Surgical removal of cerebral aneurysms before they rupture is ideal, with procedure 
mortality rates around $1 \%$, but most aneurysms go undetected due to the lack of immediate symptoms. About half of cerebral aneurysms are asymptomatic, while $32 \%$ exhibit chronic symptoms and $17 \%$ have acute symptoms [5]. Symptoms can start appearing when the aneurysm grows large enough to put pressure on nerves and the surrounding tissue or when the aneurysm ruptures. Acute symptoms include ischemia, headaches, seizures, and cranial neuropathies. Chronic symptoms are headaches, visual deficits, weakness, and facial pains. Cerebral aneurysms are known to form from bacterial infections, atherosclerosis, and trauma to the brain, but $98 \%$ of aneurysms spontaneously form [4]. There are multiple ways to treat aneurysms once found, but detection is a limiting factor in preventing deaths caused by aneurysms.

\section{3: Previous Aneurysm Studies}

Multiple studies have used FEA to simulate hemodynamics to better understand what stimulates aneurysm formation. A FEA study by Jaclyn Alfano, et al, [6] modeled 114 cerebral bifurcations from volunteers using 3D digital subtraction angiography. Each bifurcation was simulated to find the wall shear stress and wall shear stress gradient which was compared to a literature study of intracranial aneurysm locations. They found that intracranial aneurysms favored bifurcations with high magnitudes of wall shear stress and high positive wall shear stress gradients. Their study matched findings from an animal study which showed high hemodynamic shear stress promotes aneurysm genesis [6]. Other studies had similar results. A study by Z. Kulcsár, et al., [7] performed digital subtraction angiography on three patients before and after aneurysm formation. The three patients had a medical history with aneurysms, but none were present when the study began. Computational fluid dynamics were simulated for all patients using the 
digital scans before and after aneurysm formation. The study found that the point of aneurysm formation exhibited high wall shear stress and high wall shear stress gradient. Additionally, relatively high pressure was found uniformly around the point of aneurysm genesis. Changes to mechanical stimuli was hypothesized to cause destructive cellular remodeling, leading to aneurysm formation [8].

FEA has also been used to analyze how aneurysms grow and what causes them to rupture. Contrary to how aneurysms form, multiple studies have found that low wall shear stress in the aneurysm sac is related to aneurysm growth. A study by Masaaki Shojima, et al, [9] analyzed the wall shear stress and velocity in 20 digital scans of MCA bifurcations with aneurysms present. Their findings can be seen in Figure 1.
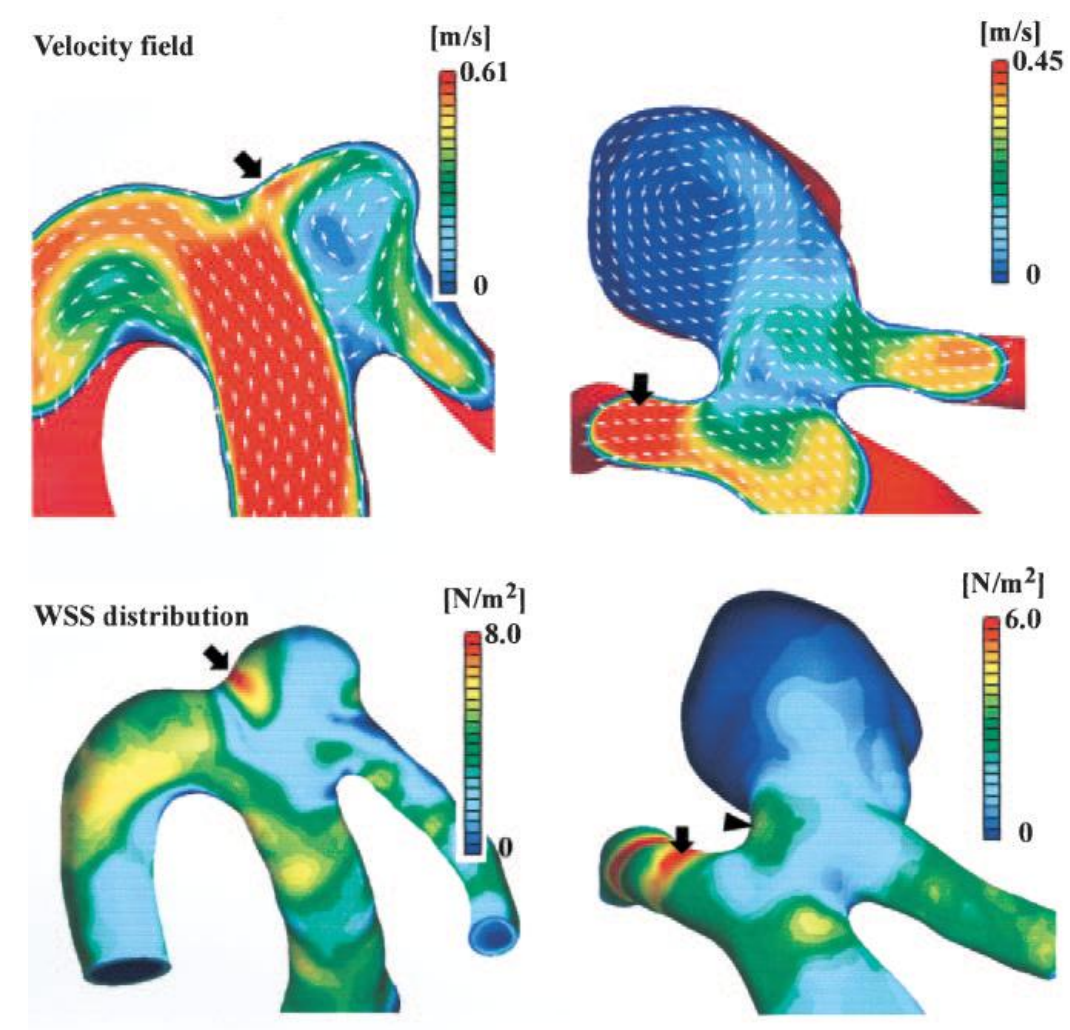

Figure 1: FEA of wall shear stress and blood velocity in a MCA bifurcation with an aneurysm [9]. 
They found that the wall shear stress in the aneurysm sac is much lower, 1.64 Pa compared to wall shear stress in the vessel region of the MCA, 3.64 Pa. The areas of low wall shear stress were typically towards the tip of the aneurysm. Additionally, the neck of the aneurysm experienced high wall shear stress, 14.39 Pa, relative to the vessel. These findings also match a study by T. Tanoue, et al, [10]. This study followed two patients with anterior communicating artery aneurysms over 27 months. One patient's aneurysm grew while the other did not. Using FEA, they found that areas of growth had extremely low wall shear stress and blood flow, $0.2 \mathrm{~Pa}$ and $0.1 \mathrm{~m} / \mathrm{s}$ respectively, while the areas of nongrowth had wall shear stress around 1.4 Pa with an average blood flow of $0.23 \mathrm{~m} / \mathrm{s}$. They hypothesized that the lack of stimulation led to degradation of the endothelial cells inside the aneurysm causing the aneurysm to grow.

Low wall shear stress is also associated with ruptured aneurysms. A study by Youchi Miura, et al., [11] found that aspect ratio, wall shear stress, normalized wall shear stress, oscillatory shear index, and wall shear stress gradient all were associated with ruptured aneurysms. They modeled and simulated fluid dynamics in 43 ruptured and 63 unruptured aneurysms using 3D rotational angiography images of intracranial aneurysms. Size of the aneurysm bulb was not found to be a factor in aneurysm rupture for aneurysms smaller than $10 \mathrm{~mm}$. Additionally, the oscillatory shear index gradient was not a factor in aneurysm rupture. After multivariate analysis, only low wall shear stress had a significant association with aneurysms that ruptured.

Vessel and aneurysm geometries are also important for designing aneurysm treatments. A study by Luciana Parlea, et al., [12] characterized the morphology of intracranial saccular aneurysms by comparing features like dome height, width, neck 
width, and semi-axis height. Intracranial aneurysms were found to be mostly spherical in shape except for anterior communicating artery saccular aneurysms, which were more pear-shaped. Most aneurysm sacs on bifurcations projected in parallel with the parent artery. The study found no morphological differences in sac features when comparing ruptured versus unruptured aneurysms.

An FEA study similar to this thesis has also been performed. A study by Michalis Xenos, et al., [13] compared different bifurcation angles and their effect on wall shear stress and pressure at the iliac bifurcation. They used an idealized model of the iliac bifurcation with a fusiform abdominal aorta aneurysm located right before the bifurcation. The study found that as the angle of the bifurcation decreased, the peak wall shear stress dropped, but the area of high wall shear stress increased. They hypothesized that the increase in area of high wall shear stress could cause additional aneurysms to form in the iliac arteries. 


\section{CHAPTER 2: BACKGROUND}

\section{1: Artery Physiology}

Arteries are blood vessels that transport blood away from the heart during rhythmic contractions of cardiac muscles which create a pressure gradient. The pressure from the heart depends on the cardiac cycle with the maximum pressure during systole, contraction of the heart, and the lowest pressure at diastole, refilling of the heart [14]. As the blood flows away from the heart, the arteries divide and get smaller. Once the arteries reach about 30 micrometers in diameter, they become arterioles [14]. Arterioles have more smooth muscle than arteries, which helps them regulate the flow of blood to various parts of the body depending on signals from the brain. This is done by contracting the smooth muscles to control the area of the lumen. Arterioles eventually become capillary beds which transfer nutrients and waste between the blood and surrounding tissue by osmosis. Blood then flows back to the heart through venules and veins.

Arteries have three unique layers to deal with the pressure from the heart. The first layer and the thinnest is the tunica intima. The tunica intima is comprised of a single layer of vascular endothelial cells that helps reduce friction as blood flows through the vessel, as well as transduce mechanical stimuli to chemical signals for the rest of the arterial layers [15]. The endothelial cells are attached to a basement membrane comprised of connective tissues and collagen bundles that anchors it to middle layer called the tunica media [16]. A layer of elastic fibers called internal elastic lamina separates the tunica intima and the tunica media. The tunica media layer provides the bulk of the mechanical properties of the artery and is comprised of smooth muscle cells, 
elastin fibers, and collagen. The smooth muscle cells allow for the vessel to constrict and modify pressure and flow through the vessel by changing the radius of the lumen. Collagen provides tensile strength, while elastin allow expansion of the vessel and dampening of the pulsatile blood flow from the heart. The external elastic lamina separates the tunica media from the outermost arterial layer, the tunica externa. The tunica externa is composed of collagen fibers that help anchor the vessel in the body, nerve fibers that stimulate the tunica media, lymphatic vessels, and blood vessels to supply nutrients to the deeper layers [15]. All layers can be seen in Figure 2 below.

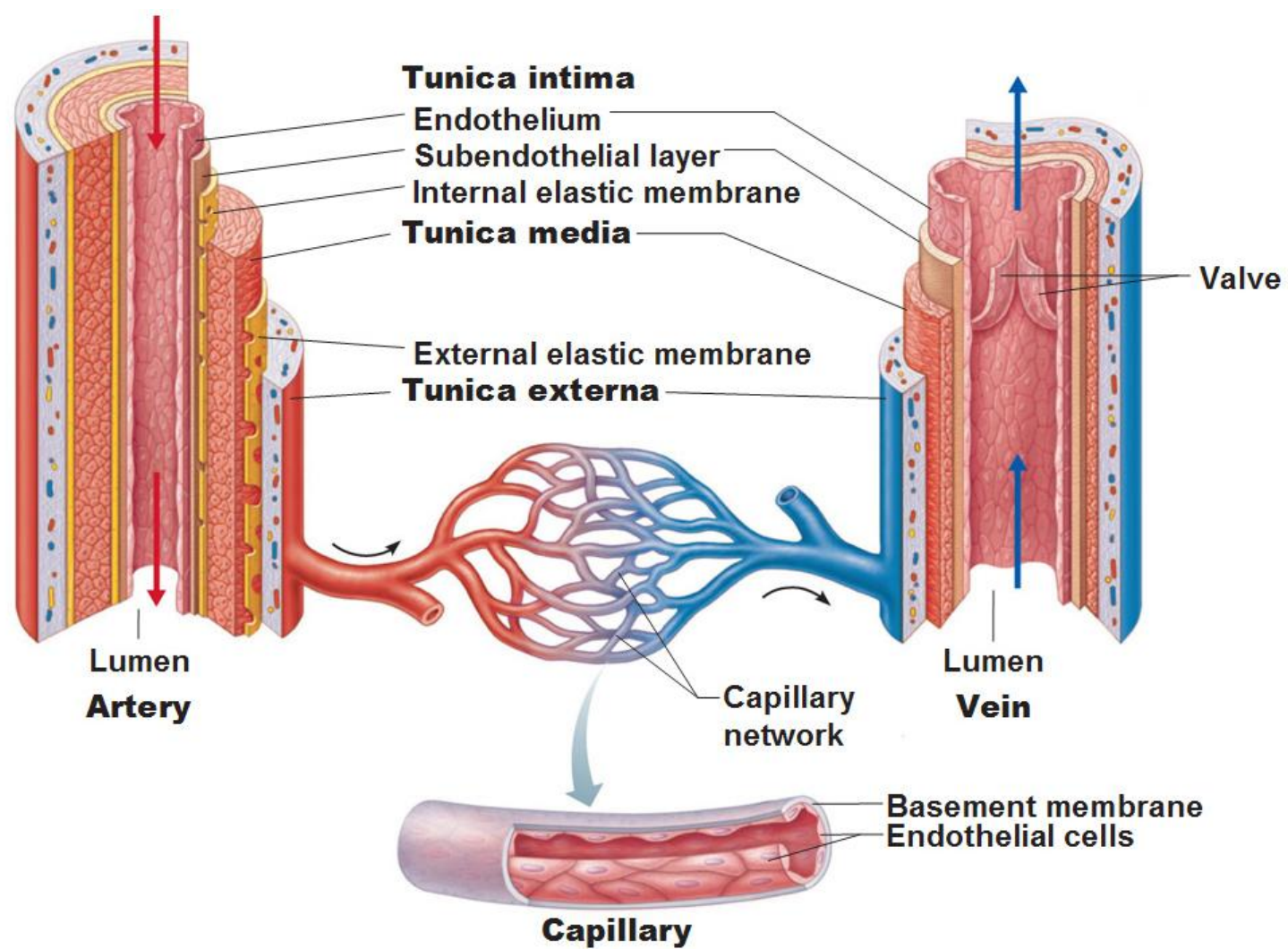

Figure 2: Diagram of the Artery Layers [14]. 
There are physiological differences depending on where the artery is in the body. Arteries closer to the heart, like the aorta, have more elastin compared to arteries further away like cerebral arteries [15]. Intracranial arteries have reduced or even missing external elastic lamina because arteries upstream from the cerebral arteries dampen the majority of the pulsatile blood flow. The smooth muscle layer is usually reduced or disorganized at bifurcations as one vessel splits into two [15]. This restructuring makes bifurcations weaker and more susceptible to damage with changes in pressure, shear stress, and flow rates. The apex of bifurcations was once thought to be weaker structurally, but instead consists of tight bands of collagen fibers causing it to be more stable [4]. One rat study found that early stage aneurysm growth occurred distal to the apex of the bifurcation, with only later stages involving the apex of the bifurcation [4].

\section{2: Aneurysm Physiology and Pathology}

Aneurysm comes from the Greek word "aneurusma", "ana" meaning throughout and "eurus" meaning wide [15]. Aneurysms are the abnormal permanent expansion of either artery or vein walls. They are typically found in arteries of people over 50, but it is not uncommon for aneurysms to be found in younger people and in veins. Aneurysms form rapidly over the course of a month where the vessel wall is rapidly remodeling [17]. The chance of rupture during vessel remodeling is high regardless of the aneurysm's final size [17]. Once an aneurysm forms, it is classified into two types, fusiform and saccular. Fusiform aneurysms are spindle shaped and are commonly located before and after bifurcations in the abdominal aorta, iliac arteries, and popliteal arteries of the knee [15]. Saccular aneurysm are small balloons/berry shaped and are normally found at arterial bifurcations and sharp curves [15]. Because of the shape, saccular aneurysms are also 
commonly referred to as berry aneurysms. The carotid artery and the Circle of Willis are common locations for saccular aneurysms to form in the brain. This thesis will be focusing on saccular aneurysms because they are the more prevalent aneurysm found at the MCA bifurcation. Figure 3 gives an example of what the two types of aneurysm look like.

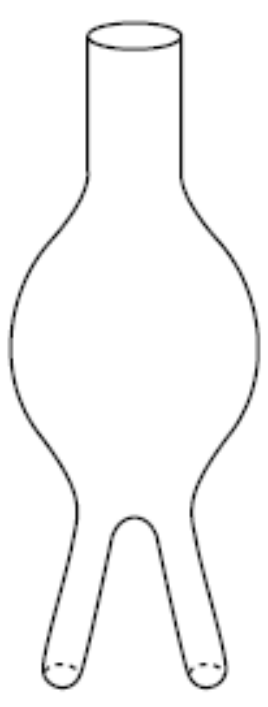

Fusiform

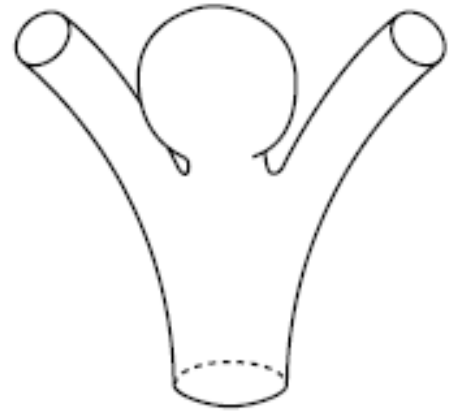

Saccular

Figure 3: Diagram of Fusiform and Saccular aneurysms [15].

Aneurysms are further classified by diameter or size. Saccular aneurysms are classified using the maximum diameter of the aneurysm bulb with small being less than $10 \mathrm{~mm}$, large between $10-25 \mathrm{~mm}$, and giant being greater than $25 \mathrm{~mm}$ [18]. Over half of the aneurysms that are treated are considered small and a quarter being large [18]. Aneurysms are also classified by location with a focus on the parent artery and angiographic features. Other metrics that are tracked are the growth rate of the bulb, aneurysm neck diameter, shape of the bulb, life style of the patient, and number of 
aneurysms found. All of these classifications give insight into the chance of rupture and how to treat the aneurysm.

Aneurysms are usually accidentally found when looking for other vascular or cerebral diseases. Saccular aneurysms that are not related to bifurcations or branching arteries are found primarily in the internal carotid artery, ICA [18]. 64\% of aneurysms that are treated on branching arteries are found on the ophthalmic and posterior communicating artery that branches from the ICA [18]. The most treated bifurcations for saccular aneurysms are the MCA, the anterior communicating artery, and ICA bifurcations with a $40 \%, 29 \%$, and $15 \%$, respectively, chance of incidence [18]. The chance of finding an aneurysm in a side branch or at a bifurcation is equal [18]. The common techniques for finding aneurysms are computed tomography (CT), magnetic resonance imaging (MRI), CT angiography, and MR angiography. These imaging techniques cannot reliably detect saccular aneurysms smaller than $2-3 \mathrm{~mm}$ in diameter, however [15]. For more precise measurements of an aneurysm, a more invasive technique called subtraction angiography is used, but it is slowly being replaced with improvements to CTA and MRA technology [15].

Once found, aneurysm treatment falls under two categories. Aneurysms under $10 \mathrm{~mm}$ are treated with medication, but aneurysms over $10 \mathrm{~mm}$ in diameter often require surgical removal [17]. Additionally, if the aneurysm has ruptured or is growing at a rate of $0.90 \mathrm{~mm}$ or greater per year, surgical removal is recommended regardless of size [5]. Saccular aneurysms have two main surgical options, packing or surgical clipping. Packing uses various materials to fill the aneurysm sac. The packing material limits blood flow into the aneurysm and causes a stable clot to prevent additional blood flow 
into the aneurysm [19]. The most common material used is platinum coils, but polymers, glue, and hydrocoils are in use, too [15]. This method works well for smaller aneurysms and is minimally invasive due to the use of a catheter. Surgical clipping is much more invasive, but can be used on aneurysms of any size, ruptured or unruptured. Surgeons place metal clips and staples at the aneurysm's neck cutting off blood flow into the aneurysm [20]. An example of aneurysm clipping can be seen in Figure 4.

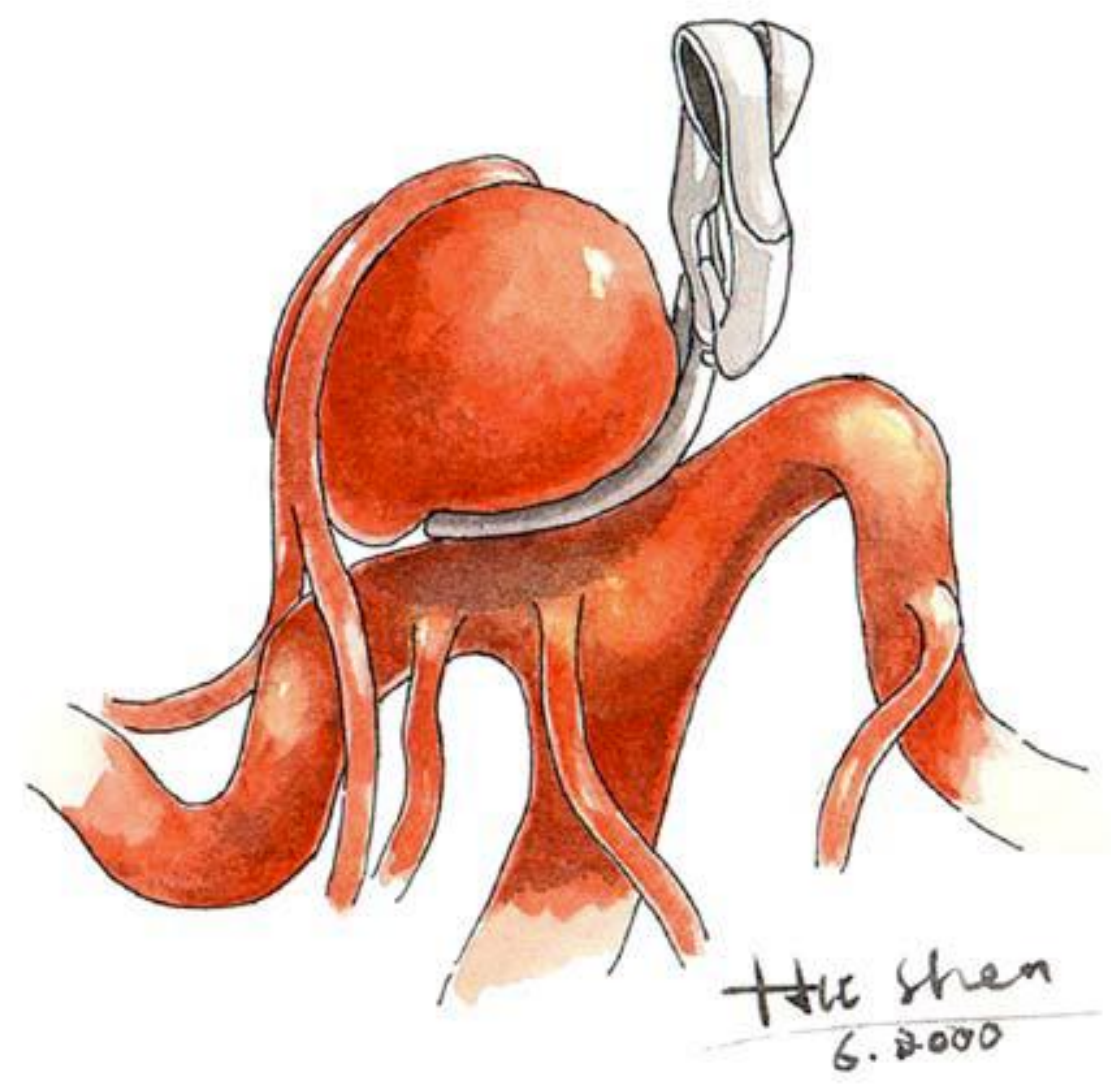

Figure 4: Example of surgical clipping of an aneurysm at a bifurcation [20].

Surgical clipping stops blood flow into the aneurysm and allows for it to clot. Aneurysms can also be clipped and then dissected out if clotting is not viable. The body will heal the vessel where the clipping occurred leaving a healthy blood vessel. Once 
removed, the chance of additional aneurysm formation or the regrowth of the removed aneurysm is very low, $1.8 \%$ and $0.52 \%$ respectively [5].

How and why aneurysms form is still unknown. It is known that alcohol consumption, smoking, and high blood pressure are risk factors for aneurysm genesis [21]. Originally, doctors thought the main cause of aneurysms was from bacterial infections of the vessel wall. Aneurysms were commonly found in patients with syphilis, heart valve infections, and infections from bloodletting. With better antibacterial drugs, aneurysm formation from bacterial infection has dropped significantly [15]. Gene disorders affecting the collagen in the blood vessels have also been known to cause aneurysms. Two examples are Marfan's and Ehlers-Danlos's Syndromes which cause abnormalities in the syntheses of elastin and collagen leading to lower mechanical properties [15]. Aneurysms also seem to have genetic predisposition, but additional studies are required [22]. Only a fraction of aneurysms come from bacteria and gene disorders, with the main etiology currently unknown. The two leading hypothesizes for aneurysm genesis are the flow shear mediated hypothesis and the fatigue/remodeling hypothesis. The shear mediated hypothesis proposes that specific fluid flow patterns and shear stresses alter the normal signaling pathways that maintain homeostasis. The stimuli change can be lower or greater than normal, as long as it is not damaging the vessel. As a person ages, the vessels remodel over time, and various formations form that disturb normal blood flow. The changes in stimuli cause destructive remodeling that causes vessel wall thinning leading to aneurysm formation. Fatigue/remodeling hypothesis instead focuses on failure of the vasculature structural proteins due to increased cyclic stresses. The high cyclic stress leads to fatigue damage and reorganization of the 
structural proteins into less stable configurations. Regardless of the pathway that triggers remodeling, the end result is thinning of the smooth muscle layer and the plastic expansion of the vessel wall.

Once the aneurysm forms, it will either stabilize or continue to grow until it ruptures or is surgical removed. One theory for saccular aneurysm growth is multiple permanent vortices inside the sac stimulate the vascular endothelial cells to cause the aneurysm to grow. Another theory is pressure inside the aneurysm sac is the major contributor to aneurysm growth with the tensile stress in the walls stimulating aneurysm growth [15]. Predicting when an aneurysm will rupture after growing is better understood than aneurysm growth. The aneurysm will rupture when the internal stress on the wall are greater than the yield strength. Computer models have been developed to determine when an aneurysm will rupture under idealized geometries and conditions [9]. The inability to determine precise geometries and vessel wall properties limits the use of these models for rupture analysis in clinical applications. Another approach is to compare aneurysms that ruptured versus those that do not. Studies have found that aneurysms with lower shear stress and a higher oscillatory shear index rupture more often [11].

\section{3: Middle Cerebral Artery Bifurcation Characterization}

The middle cerebral artery (MCA) is part of the giant circulatory anastomosis in the brain called the Circle of Willis. The MCA is comprised of four different segments identified as the M1, M2, M3, and M4. The MCA originates from the internal carotid artery (ICA) on its respective side. The ICA bifurcates to create the M1 segment of the MCA and the anterior cerebral artery. The M1 segment then bifurcates into the superior 
and inferior M2 segments. Superior and inferior refer to the orientation of the arteries rather than the physical size. This is often called the main MCA bifurcation, but can potentially trifurcate or quadfurcate. The M2 segments then bifurcate into the M3 segments, which further divides to create the M4 segments. A diagram showing the Circle of Willis and side branches of the cerebral arteries can be seen below in Figure 5. Figure 6 shows a dissected cadaver MCA bifurcation. 


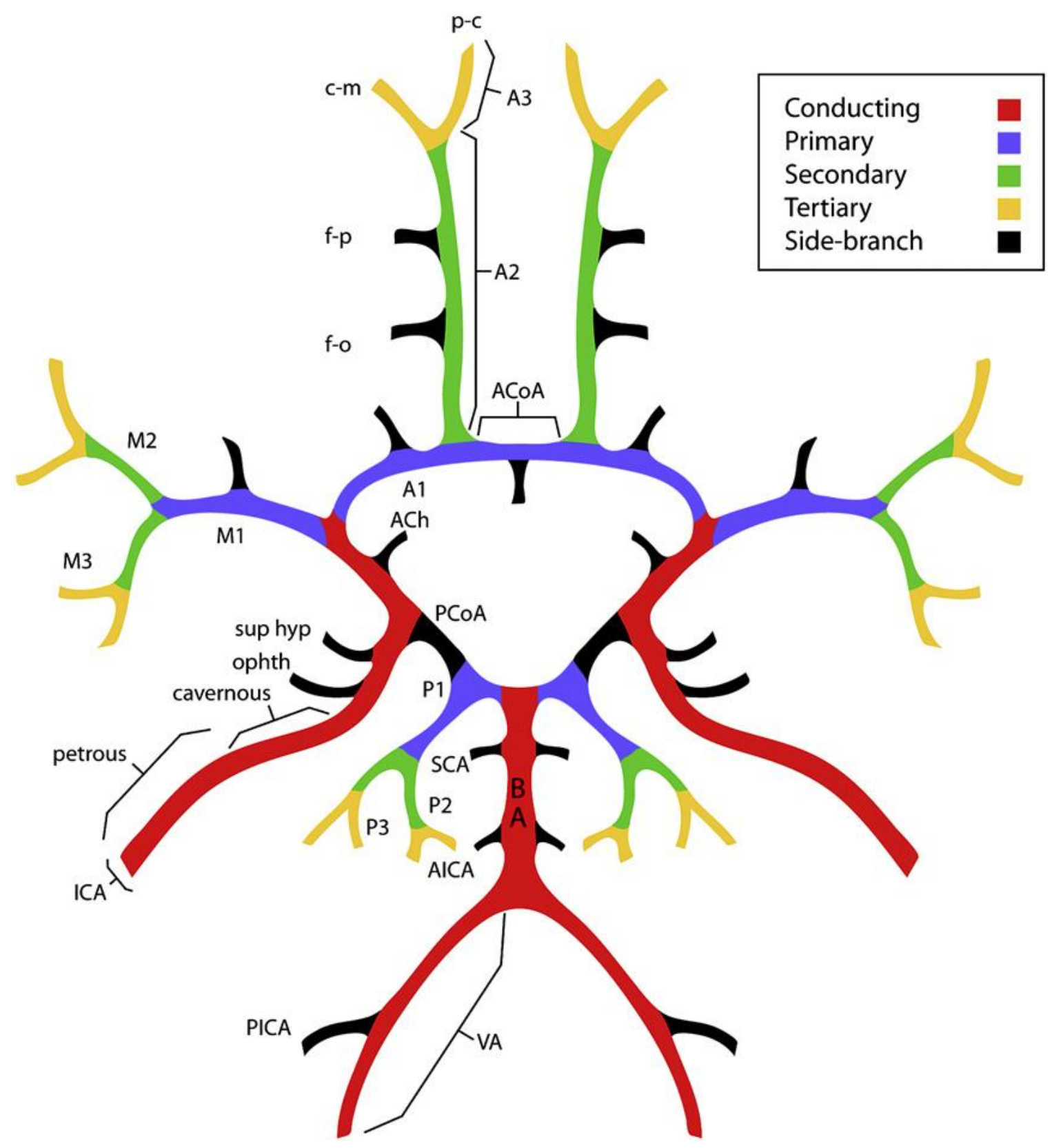

Figure 5: Diagram of Cerebral Arteries [18]. The area of interest is the main MCA bifurcation located in the middle left side of the diagram. 


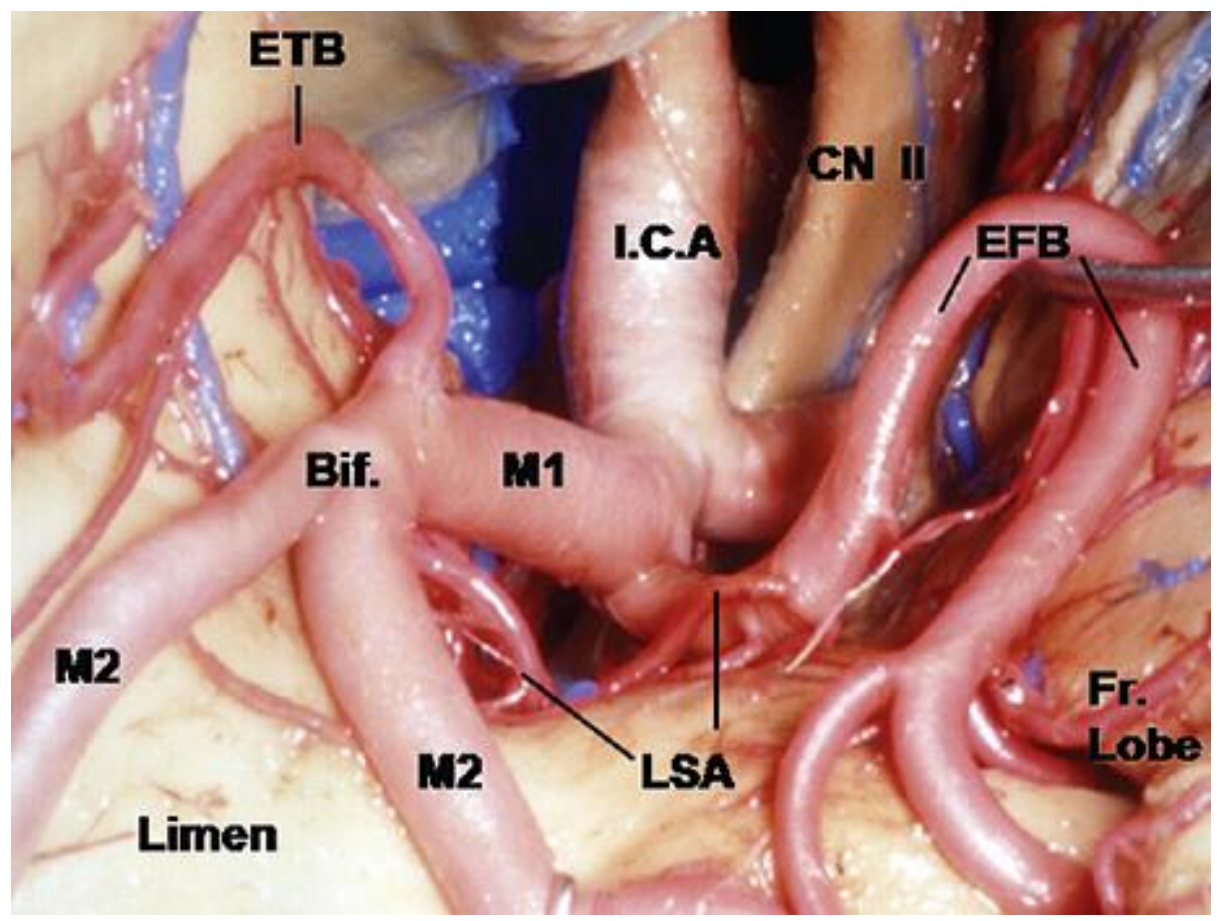

Figure 6: Surgical dissection of MCA bifurcation [23].

The area of interest is the main bifurcation of the MCA due to the likelihood of finding aneurysms. Like most biological structures, the dimensions and geometries vary greatly from person to person. The M1 segment has been measured to have a length of $20 \pm 8 \mathrm{~mm}$ with the M2 segment having a mean length of $12.1 \mathrm{~mm}[24,25]$. The inner diameter of the M1 varies from $1.9 \mathrm{~mm}$ to $3.3 \mathrm{~mm}$ with the inner diameter of the M2 branches ranging from $1.6 \mathrm{~mm}$ to $2.5 \mathrm{~mm}[1,25,26]$. The difference in diameter between the M2 segments is called the daughter artery (DA) ratio which is the M2 segment with the larger diameter divided by the M2 segment with the smaller diameter [1]. Healthy DA ratios are around $1.4 \pm 0.4$ with the inferior M2 branch being slightly larger than the superior M2 [1]. The larger daughter artery is generally $60-80 \%$ smaller than the M1 segment. There is no statistical difference regarding diameter and lengths between the left and right MCA [1]. 
Another defining characteristic of the main MCA bifurcation is the angle at which the daughter, M2, arteries bifurcate from the parent, M1, artery. In healthy individuals, the superior M2 branch has an average bifurcation angle of $117.1 \pm 22.1^{\circ}$ and the inferior M2 branch has an average angle of $107.2 \pm 24.9^{\circ}$ [1]. In healthy arteries, the LA ratio is $1.3 \pm 0.5$ [1]. The larger diameter M2 branch usually has a greater branch angle, but symmetrical branch angles and asymmetrical branch angles with the smaller daughter artery having the greater angle are possible. Again, no statistical difference has been found between the left and right MCA. Figure 7 shows a healthy main MCA bifurcation.

$\mathbf{a}$

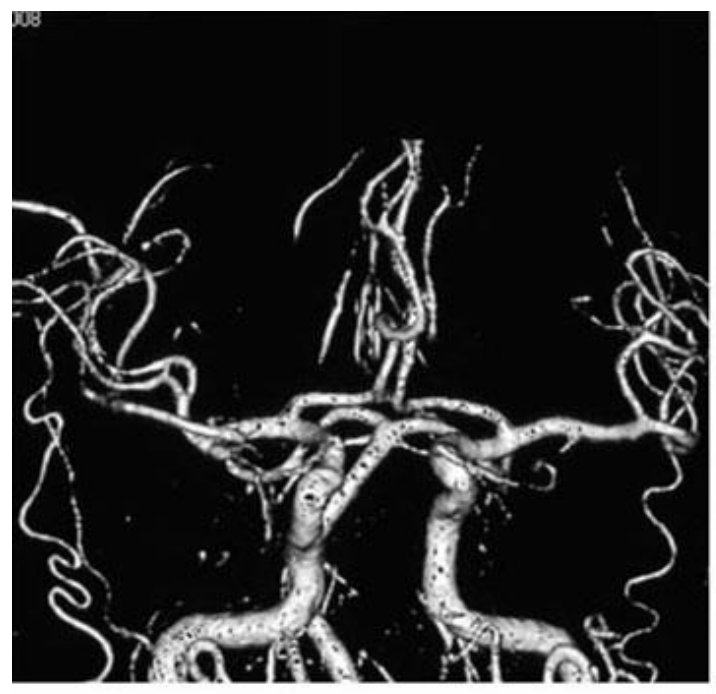

65-year-old male with right normal MCA

LA ratio: 1.0

DA ratio: 1.0
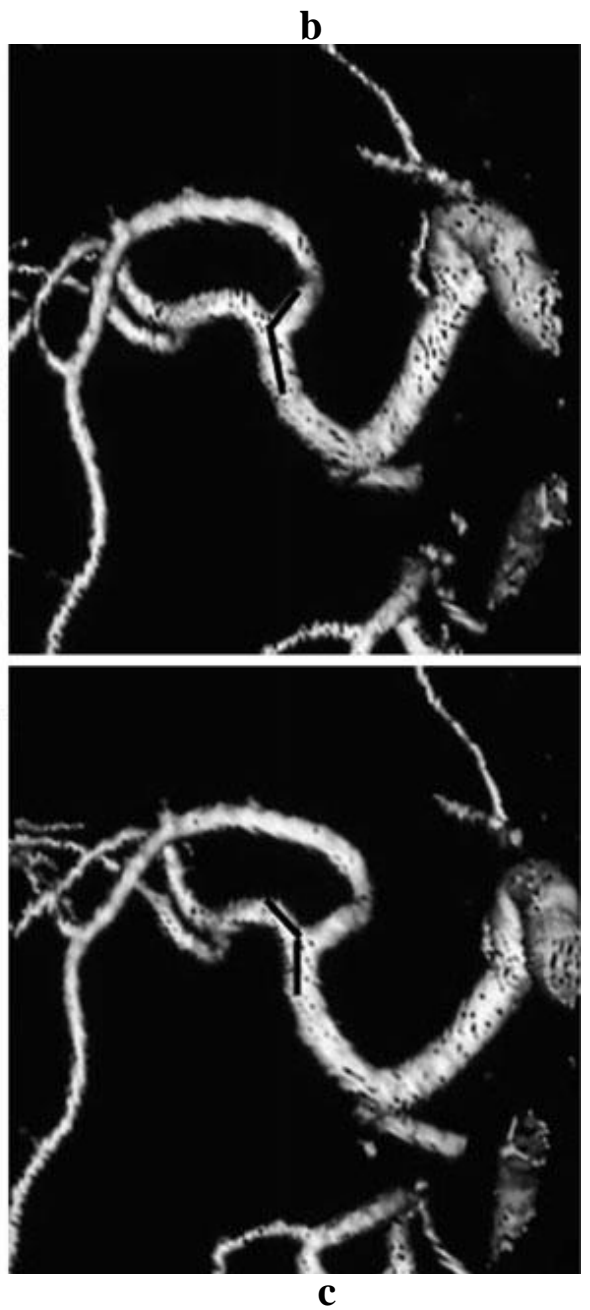

Figure 7: Representation of healthy LA and DA ratios [1]. (a)The entire cerebral vasculature. ( $b$ and $c)$ Lateral angle measurements. 
Aneurysms on the other hand have much different averages for the superior lateral angle, inferior lateral angle, and the LA ratios. In the main MCA bifurcations with aneurysms, the superior M2 branch has an average angle of $92.3 \pm 33.6^{\circ}$ and the inferior branch has an average angle of $93.2 \pm 38.3^{\circ}$ [1]. The LA ratio increases to $2.2 \pm 1.4$, indicating the chance of finding an aneurysm increases as asymmetry increases [1]. An example of diseased MCA bifurcation geometries can be seen in Figure 8.
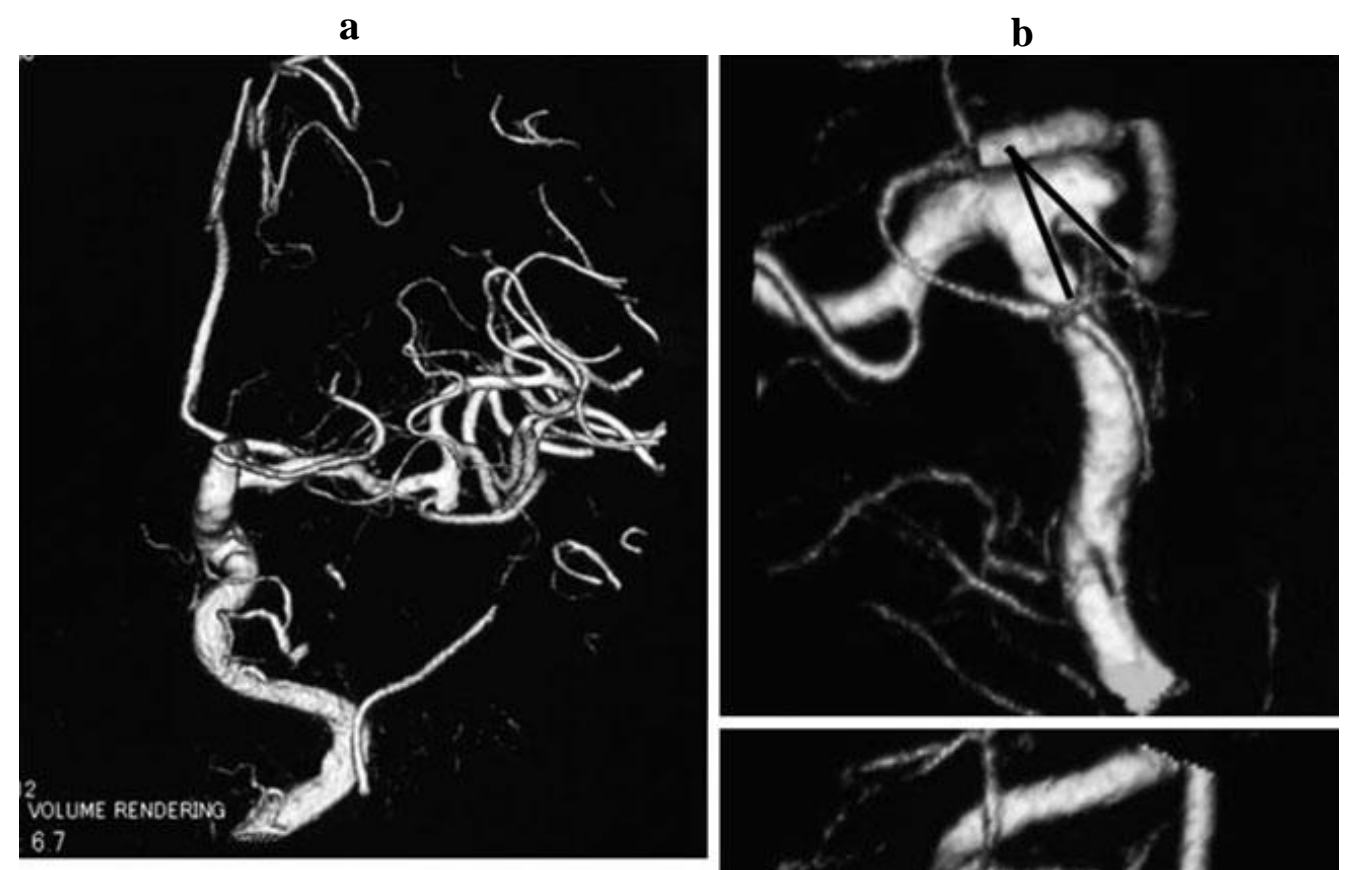

65-year-old female with unruptured aneurysm

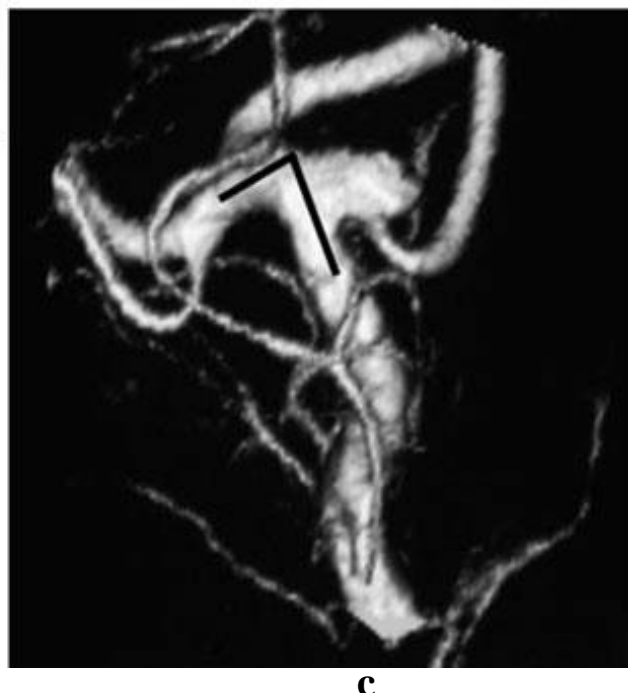

LA ratio: 2.7

DA ratio: 2.5

c

Figure 8: Representation of a MCA bifurcation with an aneurysm [1]. (a)The entire cerebral vasculature. ( $b$ and $c)$ Lateral angle measurements. 
The aneurysm in Figure 8 can be found on the right side of the bifurcation. The cut-off LA ratio for aneurysm formation was 1.6, where the likelihood of finding an aneurysm was greater than not finding an aneurysm. The DA ratio for MCA bifurcations with aneurysms increased to $1.7 \pm 0.7$, but there is no correlation between the DA ratio and aneurysm genesis. However, DA ratios lower than 1.7 are correlated to ruptured aneurysms [1].

\section{4: Loading Conditions}

A healthy adult heart beats around $68 \pm 11$ beats per minute (BPM) [27]. Heart rates can vary depending on the person, the physical activity being performed, or the position of the body. Each heart beat generates a pressure gradient that pumps the blood through the body with an average blood pressure around 120 over 70 for humans [14]. The first number, 120, represents the systolic pressure or peak pressure of the heart in $\mathrm{mmHg}$. The 70 represents the diastolic pressure or the lowest pressure of the cardiac cycle in $\mathrm{mmHg}$. The pressure in the MCA is lower due to elevation and distance from the heart. This gives the MCA a uniform $15 \mathrm{mmHg}$ decrease in pressure giving a range of 55 to $105 \mathrm{mmHg}$ over the cardiac cycle [28].

Each heart beat also expels a quantity blood called the stroke volume. The stroke volume for adults is around $70 \mathrm{ml}$ per beat, or $4800 \mathrm{ml} / \mathrm{min}$ [14]. The stroke volume can vary depending on ventricle contraction strength, preloading, heart size, and duration of contraction. Of the $70 \mathrm{ml}$ per beat, only a small fraction reaches the middle cerebral artery. The average flow rate though the MCA ranges from $121 \mathrm{ml} / \mathrm{min}$ to $158 \mathrm{ml} / \mathrm{min}$ [29]. The flow rate can fluctuate depending on age, body position, exercise, or vascular diseases. The MCA can experience an average flow rate up to $6.0 \mathrm{ml} / \mathrm{s}$ during peak 
systole and a low of $1.7 \mathrm{ml} / \mathrm{s}$ during diastole [30]. Depending on the diameter of the middle cerebral artery, velocity ranges can be as low as $23 \mathrm{~cm} / \mathrm{s}$ and as high as $106 \mathrm{~cm} / \mathrm{s}$ $[29,31]$.

Various tests can be performed to quantify blood flow. Some are noninvasive like MRI and Doppler ultrasound while others are invasive like subtraction angiography. By knowing some of the variables like pressure, size of the vessel, or flow rate, mathematical equations can be used to accurately approximate blood flow and solve for missing variables. One such equation is the Hagen-Poiseulle equation, equation 1, below, that uses pressure drop and area of the lumen to determine flow rate [32].

$$
Q=\frac{\pi R^{4}\left(P_{0}-P_{L}\right)}{8 \mu L}
$$

$\mathrm{R}$ is the inner radius of the blood vessel, $\mathrm{P}_{0}$ and $\mathrm{P}_{\mathrm{L}}$ are pressures at the opening and end, $\mu$ is the dynamic viscosity of the fluid, $\mathrm{Q}$ is the volumetric flow rate, and $\mathrm{L}$ is the length of the vessel. The Hagen-Poiseulle equation assumes that the flow is laminar, Newtonian, and flowing through a straight, rigid pipe, which are reasonable assumptions for most arterial segments [32]. Greater pressures are required to achieve similar flow rates in curved arteries.

Similar to equations that model blood flow, there are equations that model the pressure waveform of the cardiac cycle. The Windkessel model is one the oldest models, developed in the 1900s by German physiologist Otto Frank, and models the heart and vasculature using electrical circuit theory [33]. The heart is a pressure pump and is modeled as a voltage source. Blood flow is modeled as current and blood vessels are 
modeled as wires and resistors. The Windkessel model assumes that blood vessels have the ability to store fluid leading to the equation 2 below [34].

$$
Q_{\text {in }}=\frac{d V}{d t}+Q_{\text {out }}
$$

$\mathrm{Q}$ is the flow rate in and out of the blood vessel and V is the volume of stored blood due to the compliance of the vessel. Compliance is the ability to yield elastically and stretch creating a larger lumen based on vessel pressure. Each vessel has its own compliance based on the amount of elastin and other structural proteins and is modeled as a capacitor. The simplest of the Windkessel models is the 2-Element circuit seen in Figure 9.

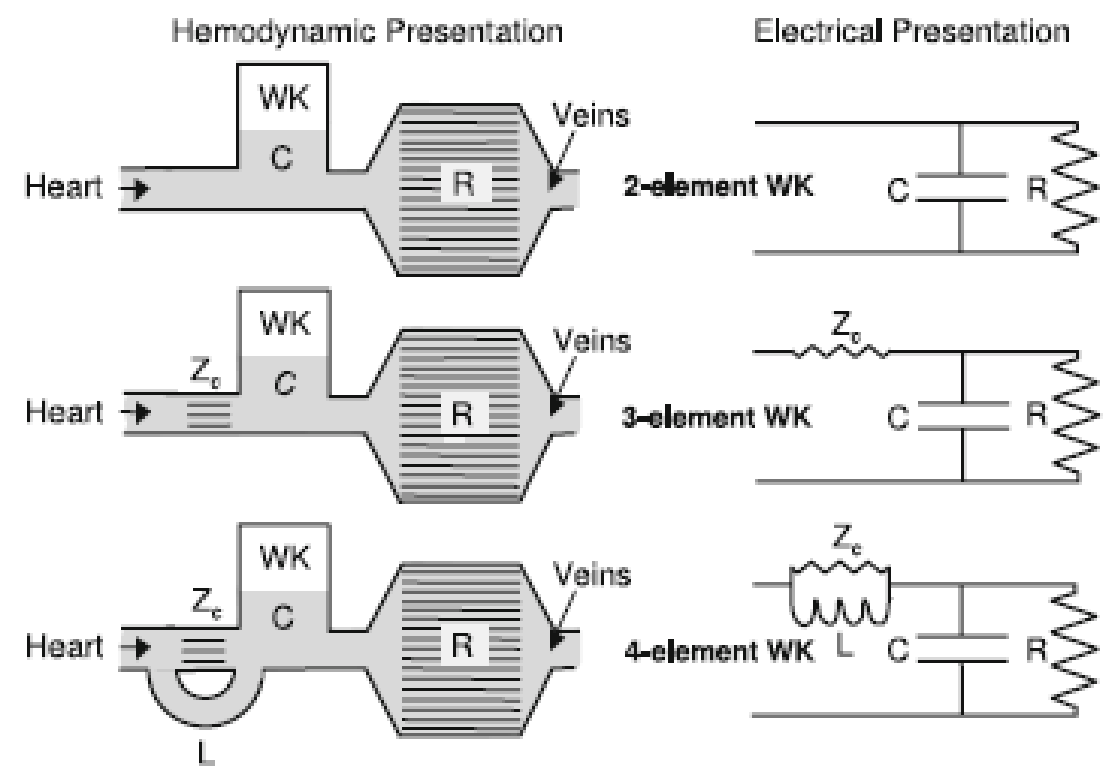

Figure 9: Two, Three, and Four Element Windkessel Model Diagram [33]. 
The 2-element model consists of a capacitor for the compliance of the vessel and a resistor for the resistance to flow caused by peripheral vasculature. Solving this circuit for the flow rate, I(t), for a 2-element Windkessel model yields equation 3 [34].

$$
I(t)=\frac{P(t)}{R}+C \frac{d P(t)}{d t}
$$

$\mathrm{R}$ is the peripheral resistance in $\mathrm{mmHg}{ }^{*} \mathrm{~s} / \mathrm{ml}$ and $\mathrm{C}$ is the compliance of the vessel in $\mathrm{ml} / \mathrm{mmHg}$. The Windkessel model splits the cardiac cycle into two parts, one for systole and one for diastole. The electrical circuit is solved normally during systole. During diastole, the voltage source is removed leaving an open circuit, causing the capacitor to release its stored power. The 2-element model is generally not used anymore since it has a poor waveform accuracy when approximating the systole relationship between flow rate and pressure. The 3 -element model fixes this by adding a second resistor to the beginning of the circuit as seen above in Figure 9. The 4-element model adds an inductor in parallel to the first resistor in the 3-element model and helps increase accuracy at frequency lower than 10hz. Figure 10, below, shows a previous study comparing measured arterial pressure in the aorta to a simulated 3-element Windkessel model 


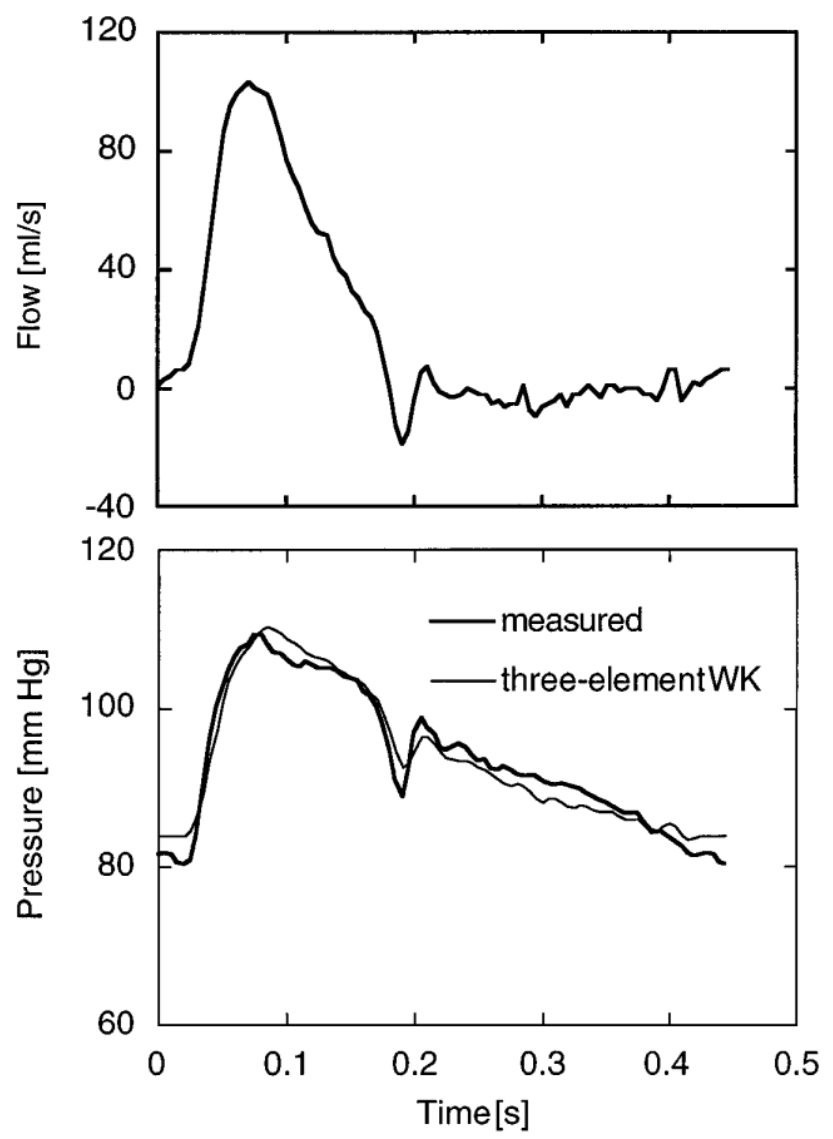

Figure 10: Graph comparing the 3-element Windkessel model to measured pressure in a human aorta [35].

Adding additional elements brings the simulated model closer to measured pressures. The 3-element does a good job of matching the overall shape of the pressure waveform given the appropriate flow rate, resistances, and compliance. The 3-element model has some error when the changing between systole and diastole.

Matlab code created by Mathew Howe simulating the 2, 3, and 4-element models was used to generate the pressure and flow rate waveforms for the middle cerebral artery. Mr. Howe's code used the 4-element Windkessel model to solve for the pressure waveform in the aorta, given the flow rate and vasculature properties. Howe's code split the Windkessel into two different equations, one for systole and one for diastole. His 
code used the ordinary differential equation (ODE) seen below, equation 4, to solve for the systole portion of the cardiac cycle [36].

$$
\left(1+\frac{R_{0}}{R}\right) Q+\left(C R_{0}+\frac{L}{R}\right) \frac{d Q}{d t}+L C \frac{d^{2} Q}{d t^{2}}=C \frac{d P}{d t}+\frac{P}{R}
$$

$\mathrm{R}_{0}$ is the proximal resistance of conducting arteries, $\mathrm{R}$ is the peripheral resistance of the capillary and veins, $\mathrm{C}$ is the capacitance of the blood vessel, and $\mathrm{L}$ is the inductance. After systole, the heart valve closes and the flow rate plus its derivatives become zero. This creates an open circuit leading to the discharge of the capacitor. Solving the new circuit gives equation 5 that models the pressure during diastole [36].

$$
P(t)=P\left(t_{D}\right) e^{-\frac{t-t_{D}}{R C}} \quad t_{D}<t<T
$$

$t_{D}$ is the time when diastole starts, while $\mathrm{R}$ and $\mathrm{C}$ are the same as in systole. This exponential decay starts at the same pressure as the end of systole and then drops back to the starting pressure of the cardiac cycle. An example of what the 3-element Windkessel simulation looks like can be seen below in Figure 11. 


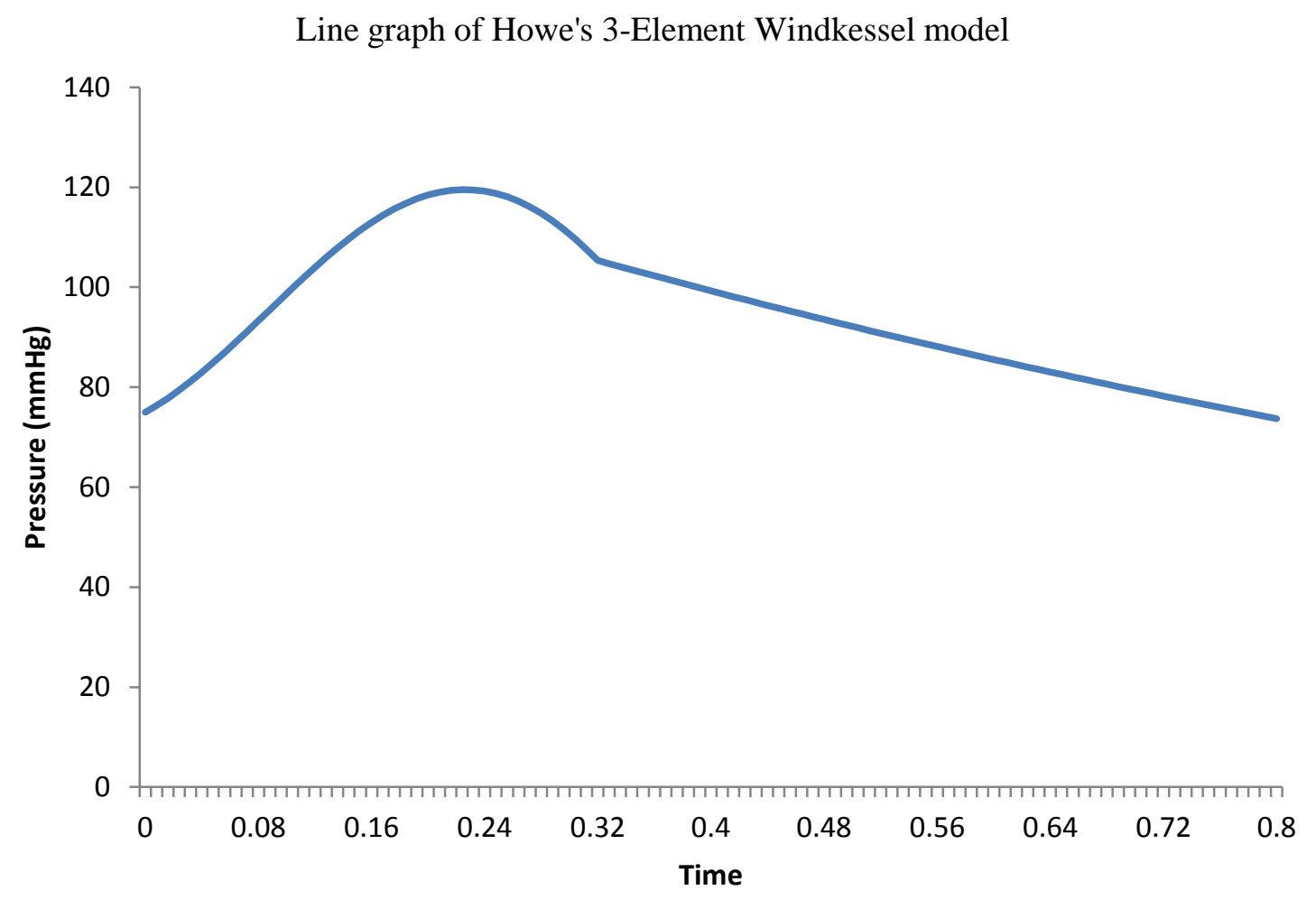

Figure 11: Example of a 3-element Windkessel model of Howe's original code for the pressure waveform in the aorta.

This waveform was generated assuming 75 beats per minute using a half sine wave as the flow rate. The variables used were $\mathrm{R}=0.80, \mathrm{R}_{0}=0.05, \mathrm{C}=1.75$ [36]. The end of systole and the beginning of diastole can be seen at 0.32 seconds.

Some modifications to Howe's code and variables were made to simulate the Windkessel model for the MCA. The 3-element model was used because of frequencies in the final model were not below $10 \mathrm{hz}$, so there would be little difference between the 3 and 4 element models. The initial settings, BPM, duration of systole, and time steps of the Windkessel simulation were left unchanged since they fell within healthy ranges for a human. BPM was 75, duration of systole was 0.32 seconds, and the number of data points for the simulation was 100 . Howe's code had a switch case statement that solved 
for 2, 3, and 4-element Windkessel models. Another switch case statement was added to allow predefined settings for pulmonary and systemic Windkessel models for the aorta. The switch case statement was hard-coded to pick the systemic 3-element Windkessel model for the aorta by setting the model_type variable to 3 and system_type variable to 1 . The variables for the hardcoded choice were then changed to simulate the MCA. The variable $\mathrm{L}$ equaled zero due to the use of the 3 -element model, while $\mathrm{R}, \mathrm{R}_{0}, \mathrm{C}$ were obtained through research.

The final alteration to Howe's code was implementing a new flow rate equation. Studies show that the velocity waveform matches the pressure waveform in the MCA [28]. Since the pressure waveform in the MCA is scaled down $15 \mathrm{mmHg}$ compared to the aorta, the pressure waveform created by Howe's code for the aorta was used as a template to create the flow rate waveform for the MCA. This was done by running Howe's original code for the systemic 3-element Windkessel model. The array for the solved pressure and time was then exported into Excel. The pressure waveform was scaled down by dividing it by 13 to achieve homeostatic flow rates. A polynomial trend line was placed on the scaled down pressure waveform to create the equation to describe flow rate. The equation can be seen in equation 6 .

$$
\begin{gathered}
Q(x)=11466.8 x^{6}-3832 x^{5}+3729 . x^{4}-1605.2 x^{3}+251.61 x^{2} \\
+5.5689 x+2.2
\end{gathered}
$$

The variable $\mathrm{x}$ represents time. The polynomial had a coefficient of determination of 0.99 indicating a good fit for the flow rate waveform. The polynomial 
was then exported into Howe's Matlab code where it replaced the flow rate in the Windkessel.m file. The polynomial and its derivatives also replaced the flow rate in Windkessel_DE.m in order for Matlab to solve the Windkessel ODE. The flow rate used for the Windkessel model and the simulated pressure waveform for the MCA can be seen in Figure 12 and Figure 13.

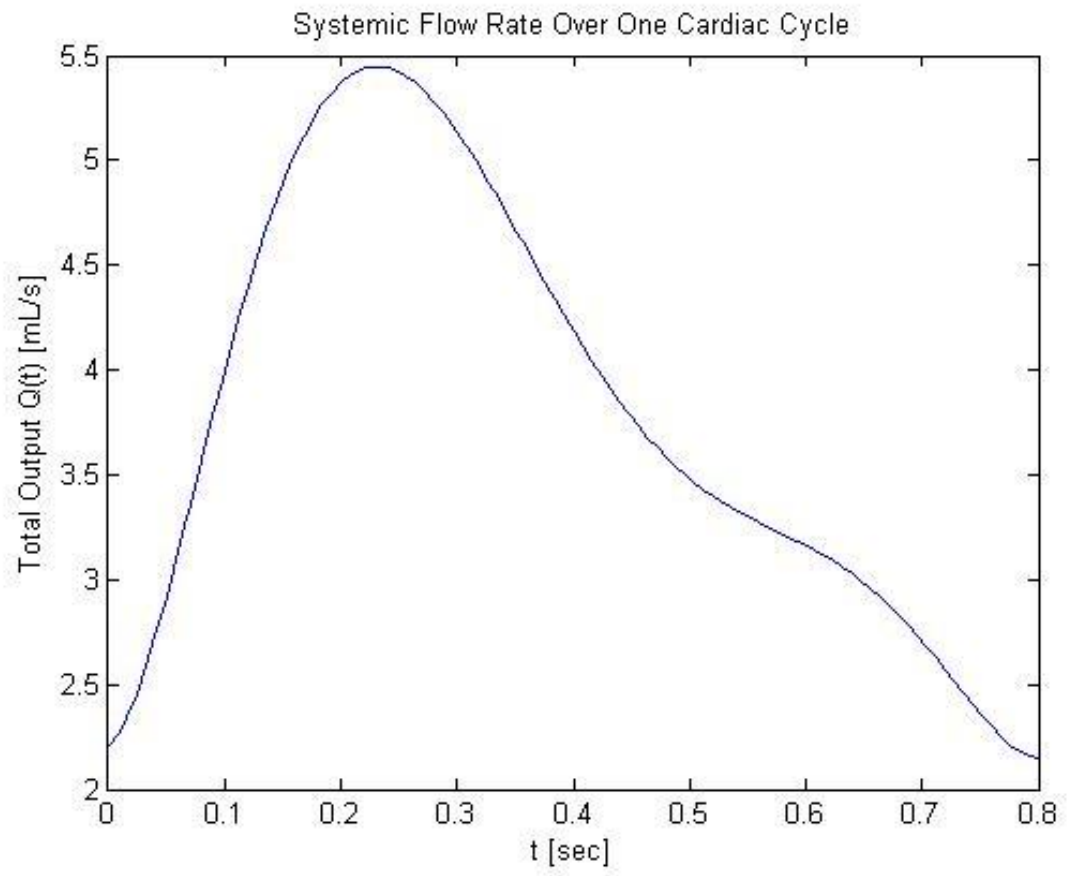

Figure 12: Flow Rate of blood in the MCA. 


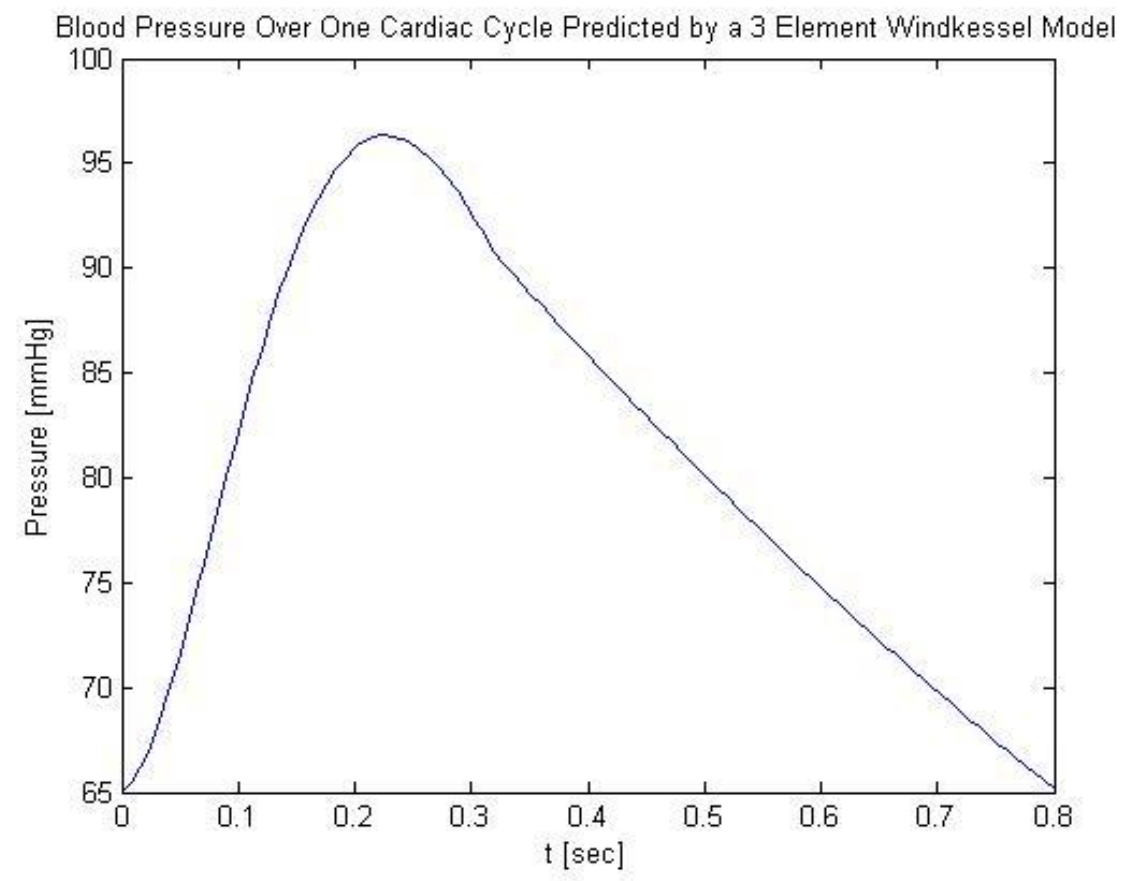

Figure 13: Simulated Windkessel cardiac pressure waveform for the MCA.

The pressure waveform seen in Figure 13 used a starting pressure of $65 \mathrm{mmHg}$ with $\mathrm{R}=5.54, \mathrm{R}_{0}=10.5, \mathrm{C}=0.265$, and $\mathrm{L}=0$ [37]. These values were obtained from literature and then adjusted to correct the pressure range and end pressure. The pressure waveform simulated with the Windkessel model for the MCA has a smaller range between diastole and systole, but still within healthy levels.

\section{5: Rheology and Shear Stress}

Blood is fluid mixture of plasma and cells. Plasma is primarily water with various proteins, electrolytes, hormones, and gases mixed in. The cells that make up blood are the erythrocytes, leukocytes, and platelets [14]. Erythrocytes, red blood cells, make up 95\% of the cell volume in the blood and the volume fraction of erythrocytes is around $40-60 \%$ [32]. Platelets make up $4.9 \%$ of the cell volume and leukocytes, white 
blood cells, are the left over $0.1 \%$ [32]. These cells, along with the proteins and ions in the plasma, give blood its unique fluidic properties. Blood has a density of $1060 \mathrm{~kg} / \mathrm{m}^{3}$, a $\mathrm{pH}$ of 7.4 , and a viscosity at high shear rates of $0.0035 \mathrm{cP}$ at $37^{\circ} \mathrm{C}$ [32]. Blood is a shear thinning, non-Newtonian, fluid. The viscosity changes depending on the velocity of the blood, with low velocities leading to higher viscosities and high velocities leading to lower viscosities. This leads to a nonlinear shear rate to shear stress relationship.

There are several different equations that model the viscosity of blood. The simplest way is to assume blood is a Newtonian fluid with a constant viscosity, usually around 3.0 to $4.0 \mathrm{cP}$ depending blood composition and temperature. This method is chosen for large arteries like the aorta where fluid flow is fast. When Newtonian viscosity isn't a valid assumption, there are multiple accurate non-Newtonian models available. The Casson equation is a empirically derived non-Newtonian model that requires a minimum force for stagnant blood to start flowing. It describes the shear stress to shear rate relationship of blood and can be rearranged into equation 7 to solve for viscosity [38].

$$
\mu=\left(\sqrt{\mu_{0}}+\sqrt{\frac{\tau_{0}}{\dot{\gamma}}}\right)^{2}
$$

$\tau_{0}$ is the yield stress of blood, $\mu_{0}$ is the Newtonian viscosity of blood, and $\dot{\gamma}$ is the shear rate. At higher shear rates, this equation will asymptotically approach the Newtonian viscosity for blood, $0.0035 \mathrm{cP}$. The Casson equation is dependent on anything that changes viscosity of blood, like protein concentration, cell volume 
fractions, and temperature [38]. The Casson equation accuracy breaks down at shear rates below $10 \mathrm{~s}^{-1}[39]$

Another model for non-Newtonian viscosity is the Power law model seen in equation 8 [40].

$$
\mu=\mu_{0} \dot{\gamma}^{n-1}
$$

$\mu_{0}$ is the Newtonian viscosity of blood, $\dot{\gamma}$ is the shear rate, and $\mathrm{n}$ is a constant. This model is particularly good at simulating blood at low velocities, around $0.025 \mathrm{~m} / \mathrm{s}$, but loses accuracy at high velocities, greater than $0.6 \mathrm{~m} / \mathrm{s}$ [40]. This model would be good for predicting viscosity in berry aneurysms, but poor for healthy arteries or arteries that feed blood flow into the aneurysm.

A good balance is the Carreau-Yasuda model for blood viscosity seen in equation 9, which works for both fast and slow fluid velocities [40].

$$
\mu=\mu_{\text {inf }}+\left(\mu_{0}-U_{\text {inf }}\right)\left[1+(\lambda \dot{\gamma})^{2}\right]^{\frac{n-1}{2}}
$$

$\mu_{\text {inf }}$ is the Newtonian viscosity of blood, $\mu_{0}$ is the zero shear rate viscosity of blood, $\dot{\gamma}$ is the shear rate, $\lambda$ and $\mathrm{n}$ are both constants related to the simulated fluid. This model accurately simulates blood viscosity at low velocities similar to the Power law model, but also matches Newtonian fluids at higher velocities [40]. This property makes the model ideal for blood flow in berry aneurysms since it can accurately simulate the viscosity of both the arteries and the aneurysm bulb in the same model. 
Modeling the viscosity accurately is important for determining the fluid flow and shear stress. Wall shear stress plays a major role in homeostasis and calculating shear stress is very important in predicting the formation and diagnosing circulatory diseases. One way to estimate the wall shear stress acting on a blood vessel is to use HagenPoiseuille equation, equation 1 , above, to estimate the flow rate and then use the relationship in equation 10 to predict the wall shear stress [32].

$$
\tau_{w}=\frac{4 \mu Q}{\pi R^{3}}
$$

This equation uses the same assumptions as Hagen-Poiseuille: laminar, Newtonian fluid and a straight rigid pipe. $\mu$ is the Newtonian viscosity of the fluid, $Q$ is the averaged flow rate in the vessel, $\mathrm{R}$ is the radius to the lumen. Changes in temperature, vessel geometry, and blood composition can lower the accuracy of this equation. Computer models can also predict shear stress, but instead use a generalized equation seen below to find shear stress anywhere in the model [41].

$$
\tau=\mu \dot{\gamma}
$$

$\mu$ is the viscosity of the fluid and $\dot{\gamma}$ is the shear rate. For Newtonian fluids, the viscosity is constant and shear stress is dependent on shear strain. For blood, the viscosity is not constant and leading to non-glinear characteristics.

To maintain the artery and its layers, the endothelial cells in the tunica intima transduce the mechanical forces into chemical signals to maintain vessel homeostasis. 
The mechanical forces the endothelial cells reacts to are pressure, circumferential stretch/tension, and shear stress. Shear stress in particular controls endothelium survival. Too little shear stress has shown to trigger apoptosis and random endothelium orientations while large shear stress damages the endothelium [42]. The endothelium also requires pulsatile shear stress for cells to proliferate and grow. It is believed that nitric oxide, NO, is the key mediator for vessel homeostasis through shear stress. NO is made by the enzyme ecNOS found in the endothelium. Figure 14 showcases some of the hypothesized pathways in which shear stress causes NO production.

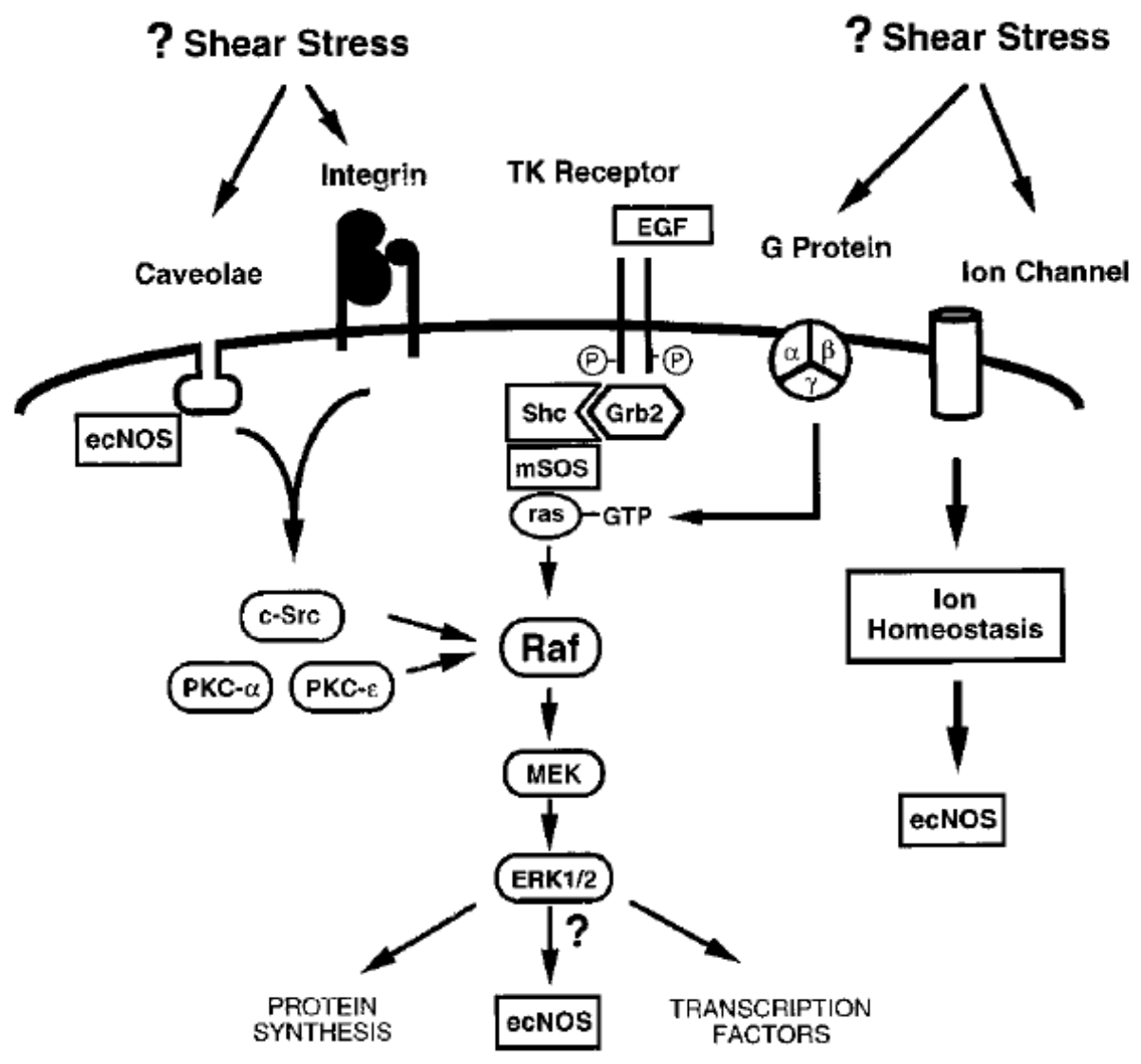

Figure 14: Hypothesized pathways for mechanical stimuli to ecNOS activation [42]. 
There are four main pathways that are believed to transduce shear stress to ecNOS activation. One pathway is through caveolae. Caveolae are areas in the plasma membrane rich in cholesterol, causing the membrane to be more rigid. Caveolae are known to interact with signaling proteins like G proteins, c-Src-family tryosine kinases, ras, and PKC family kinases [42]. Additional research is required to better understand how caveolae mechanical properties and signaling cascade lead to NO production. The second pathway is through $\alpha / \beta$ integrins, which are transmembrane glycoproteins that are adhesion receptors for cell to cell interactions. It is unknown which integrins cause the activation of ecNOS, but lab results shown that $\beta 1$ and $\beta 3$ integrins partially control NO release. Another pathway for ecNOS activation is through $\mathrm{G}$ protein. G proteins are a transmembrane grouping of proteins that transduce stimuli into chemical signaling using a cascade of protein activation. For shear stress activated $G$ proteins, the cascade is believed to stop at the phosphorylation of ras, a GTP binding protein [42]. All three of these pathways, the caveolae, integrin, and $\mathrm{G}$ proteins, are believed to activate Raf-1, a MAP kinase kinase kinase. Caveolae and integrins are thought to activate Raf-1 though the phosphorylation of PKC- $\alpha, \mathrm{PKC}-\varepsilon$, and c-Src while G proteins through ras [42]. Raf1 activates MEK-1, which activates ERK 1/2 [42]. ERK 1/2 then activates ecNOS as well as additional gene activation and the phosphorylation of transcription factors like cfos, c-jun, and c-myc [42]. The final hypothesized pathway for ecNOS activation is through mechanosensitive ion channels that respond to the mechanical shear stress from blood with the change in ion concentration in the cytoplasm leading to ecNOS activation [42]. Studies have found blocking $\mathrm{K}^{+}$ion channels stopped shear mediated increases in NO production [42]. Most likely, all these pathways are occurring at the same time with 
additional pathways that are still unknown. Additional research is needed to fully understand how mechanical stimuli causes NO production, gene activation, and transcription factors activation.

In healthy cerebral arteries, the shear stress can range from $1 \mathrm{~Pa}$ to $7 \mathrm{~Pa}$ [41]. At arterial bifurcation, however, the shear stress can be many times greater. Various computer models have estimated that the shear stress at the main MCA bifurcation averages around 18 to $22 \mathrm{~Pa}[6,43]$. One study found a peak wall shear stress of $30 \pm 5 \mathrm{~Pa}$ at the MCA bifurcations, but this study lumped all bifurcations of the middle cerebral artery together [6]. Another simulation has found a max wall shear stress of $33.17 \mathrm{~Pa}$ for a mean flow velocity of $75 \mathrm{~cm} / \mathrm{s}$ at the main MCA bifurcation using idealized geometries [44]. The exact homeostatic range for shear stress at the main MCA bifurcation is unknown, but current studies show that shear stress is higher compared to non-bifurcating arteries. 


\section{CHAPTER 3: MODELING METHODS}

\section{1: Modeling Overview}

Multiple models were created using a student license of COMSOL

Multiphysics®. COMSOL Multiphysics® is a finite element analysis (FEA)

multiphysics software suite that can simulate fluid flows, particle tracings, and

mechanical stresses. The models simulated were various bifurcation angles of the main MCA bifurcation in order to observe changes in fluid flow and wall shear stress. The geometries were created in SolidWorks ${ }^{\circledR}$ and imported into COMSOL where various post processing techniques were performed to verify the accuracy of the computer model and compare results between models.

FEA works by breaking down complex problems into smaller, easier to solve pieces. This method allows accurate approximations of complex problems using the weak forms of the governing equations to solve ordinary differential equations, partial differential equations, and linear equations. Governing equations are the equations that describe the physics being simulated. The simulation is first partitioned into many simple subdomains that are comprised of elements and nodes in the shape of pyramids, prisms, and hexahedrals. Each of these subdomains have an elemental equation that locally approximates the governing equations. The elemental equations are solved using either linear algebra or numerical integration depending on the governing equations. The subdomains are then stitched together through the nodes to recreate the original problem and boundary conditions are applied to achieve an approximate solution. Increasing the number of subdomains increases the accuracy of the simulation, but adds to the computation time of the problem. 


\section{2: Fluid Flow Module and Loading Conditions}

The first simulations created were 2D models with idealized geometries in order to set up the fluid dynamic modules in COMSOL. The models were created in COMSOL using the built in computer aided design (CAD) software. The geometries modeled were $5 \mathrm{~mm}$ long M1 parent artery with a symmetrical $120^{\circ}$ bifurcation to the two M2 daughter arteries that were also $5 \mathrm{~mm}$ long. The diameter of the parent artery was $3 \mathrm{~mm}$, the superior daughter artery, left side, was $2.475 \mathrm{~mm}$, and the inferior daughter artery, right side was $2.250 \mathrm{~mm}$ in diameter. The idealized bifurcation can be seen in Figure 15 .

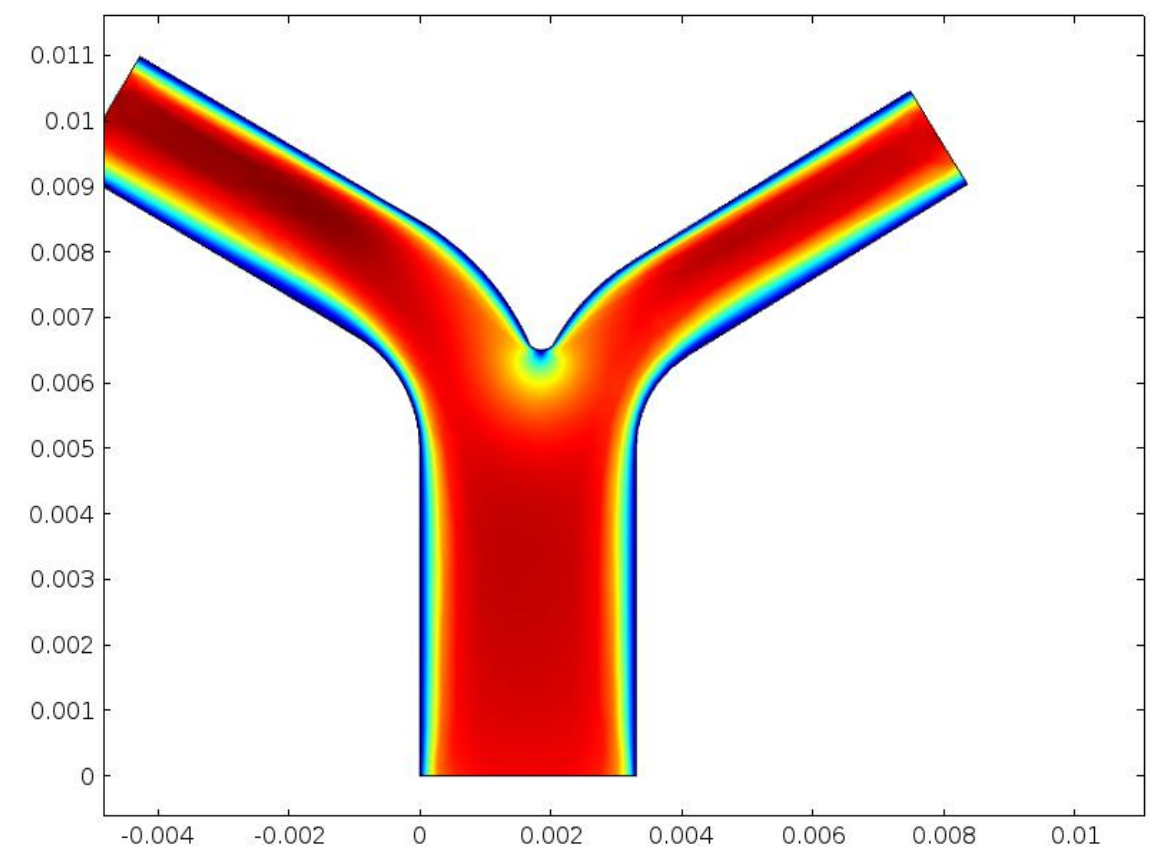

Figure 15: Velocity profile of Idealized 2D bifurcation with non-Newtonian laminar flow

This base geometry was then used to create iterative simulations starting with Newtonian creep flow. Once basic fluid motion was achieved, the model was changed to Newtonian laminar flow, then to non-Newtonian creep flow, and finally non-Newtonian 
laminar flow. All 2D models were stationary models except for the final non-Newtonian laminar flow model which was a time dependent model to allow the simulation of the cardiac cycle.

First, a material called blood was created and was given a density of $1060 \mathrm{~kg} / \mathrm{m}^{3}$ with a viscosity of $0.0035 \mathrm{cP}$, the density and viscosity for Newtonian blood at $37^{\circ} \mathrm{C}$. This material was assigned to the entire domain of the $2 \mathrm{D}$ model. The fluid properties section under creep and laminar flow had its density and viscosity set to the created blood material for both Newtonian models. For the non-Newtonian models, the density was set to the created blood material, but the viscosity was simulated using the Carreau-Yasuda model, equation 9, for viscosity. Carreau-Yasuda viscosity model was chosen because it could accurately simulate viscosity in arteries and in aneurysms, which was a future ambition for these models. The variable for Carreau-Yasuda model were set to simulate blood with $\mu_{0}=0.056 \mathrm{~Pa}^{*} \mathrm{~s}, \mu_{\mathrm{inf}}=0.0035 \mathrm{~Pa}^{*} \mathrm{~s}, \lambda=3.313 \mathrm{~s}$, and $\mathrm{n}=0.3568[40,45]$.

To simulate the fluid flow, two different fluid modules were used. The first and more simplistic module was fluid creep flow. Creep flow, also known as stokes flow, is the Navier-Stokes equation for fluid flow with one side equal to zero to yield equation 12 and equation 13 .

$$
\begin{gathered}
\bar{\nabla} \cdot\left[-\rho \overline{\bar{I}}+\mu\left(\bar{\nabla} \bar{v}+(\bar{\nabla} \bar{v})^{T}\right)\right]+\bar{F}=0 \\
\rho \bar{\nabla} \cdot \bar{v}=0
\end{gathered}
$$

The $\nabla$, del operator, is the vector differential operator, $\rho$ is the density of the fluid, I is the identity matrix, $\mu$ is the viscosity of the fluid, $v$ is the fluid velocity, $T$ is the 
transpose of a matrix, and $\mathrm{F}$ is the force acting on the fluid. Creep flow is generally used for fluids with Reynolds numbers much less than one where viscous forces dominate. The simplification of Navier-Stokes makes solving the differential equation much faster and saves on computation time at the cost of accuracy. Once the creep flow was implemented successfully, it was replaced with the laminar fluid flow module. Laminar flow uses the same Navier Stokes equation, but does not simplify it yielding equation 14 and 15 below.

$$
\begin{gathered}
\rho \frac{\partial v}{\partial t}+\rho(\bar{v} \cdot \bar{\nabla}) \bar{v}=\bar{\nabla} \cdot\left[-\rho \overline{\bar{I}}+\mu\left(\bar{\nabla} \bar{v}+(\bar{\nabla} \bar{v})^{T}\right)\right]+\bar{F} \\
\rho \bar{\nabla} \cdot \bar{v}=0
\end{gathered}
$$

Similar to creep flow, $\nabla$ is the del operator for a vector, $\rho$ is the density of the fluid, $\mathrm{I}$ is the identity matrix, $\mu$ is the viscosity of the fluid, $\mathrm{v}$ is the fluid velocity, and $\mathrm{F}$ is the force acting on the fluid. With the added terms, a nonlinear partial differential equation is created, which greatly adds to the computation time, but greatly increases the accuracy for simulating fluid with a Reynolds number less than 2000. Laminar flow module was chosen over creep flow because blood flow in arteries falls around a Reynolds number of 600 [46].

To simulate the loading conditions for the MCA, the walls of the model were set to rigid with the no slip condition, velocity at the wall is zero. Previous studies have found that the changes to hemodynamics is small when comparing rigid MCA models to elastic models [11]. The pressure waveform data created by the 3-element Windkessel model, Figure 13, was moved into excel, extrapolated to four heart beats, and then 
imported into COMSOL using the interpolation function. The pressure waveform was then placed on the inlet, the parent artery, and outlets, the two daughter arteries. The inlet was set to laminar inflow with an entrance length of $0.4 \mathrm{~m}$ to remove inlet anomalies and to allow the laminar flow profile to fully develop. The flow rate waveform used in the Windkessel model was then used with the Hagen-Poiseulle equation to create a pressure drop between the inlet and outlet that would drive the blood through the bifurcation. The radius used in Hagen-Poiseulle was adjusted to get proper flow rates to offset the equation's assumptions. The pressure drop over time was created in Excel and imported into COMSOL using the interpolation function and was subtracted from the outlet boundary condition to generate fluid flow. A time dependent study to simulate the model was made with 0.008 second time steps and simulated 4 heart beats, 3.224 seconds.

\section{3: Geometries of Model}

With the confirmation of the fluid modules working correctly the $2 \mathrm{D}$ simulation was converted into a 3D simulation. All settings for fluid flow, materials, boundary conditions, and the time dependent study were left unchanged. New idealized 3D MCA bifurcation models were made using SolidWorks. SolidWorks was used over COMSOL CAD because it could smooth the model around the bifurcation point. Three base angles, $120^{\circ}, 90^{\circ}, 60^{\circ}$, were chosen to be the angle between the M1 and the larger M2. LA ratios of 1.0, 1.6, and 2.2 were then applied to each of the base angles to create eight models. 60 degrees with a LA ratio of 1 was excluded since MCA bifurcation at that angle never exhibited symmetrical bifurcation angles. The parent artery was $3 \mathrm{~mm}$ in diameter and both M2 daughter arteries were $2.12 \mathrm{~mm}$ in diameter. The daughter arteries are the same diameter to remove any effects they might have on fluid flow and wall shear stress. 
Without this, fluid flow would change and bias a side for higher flow rates, leading to changes in wall shear stress not related to angle geometries. The model was created by making a line outline of the desired bifurcation angle. The outline was seeded with multiple planes perpendicular to the lines and circles representing vessel diameters were add to each plane. The loft function was used to connect each circle to create the bifurcation. The bifurcation apex was then chamfered to complete the model. Figure 16 and Figure 17 below shows the model with the base angle 120 with a LA ratio of $1\left(120^{\circ}\right.$ $120^{\circ}$ ) that was used as a control for verification and comparison between models.

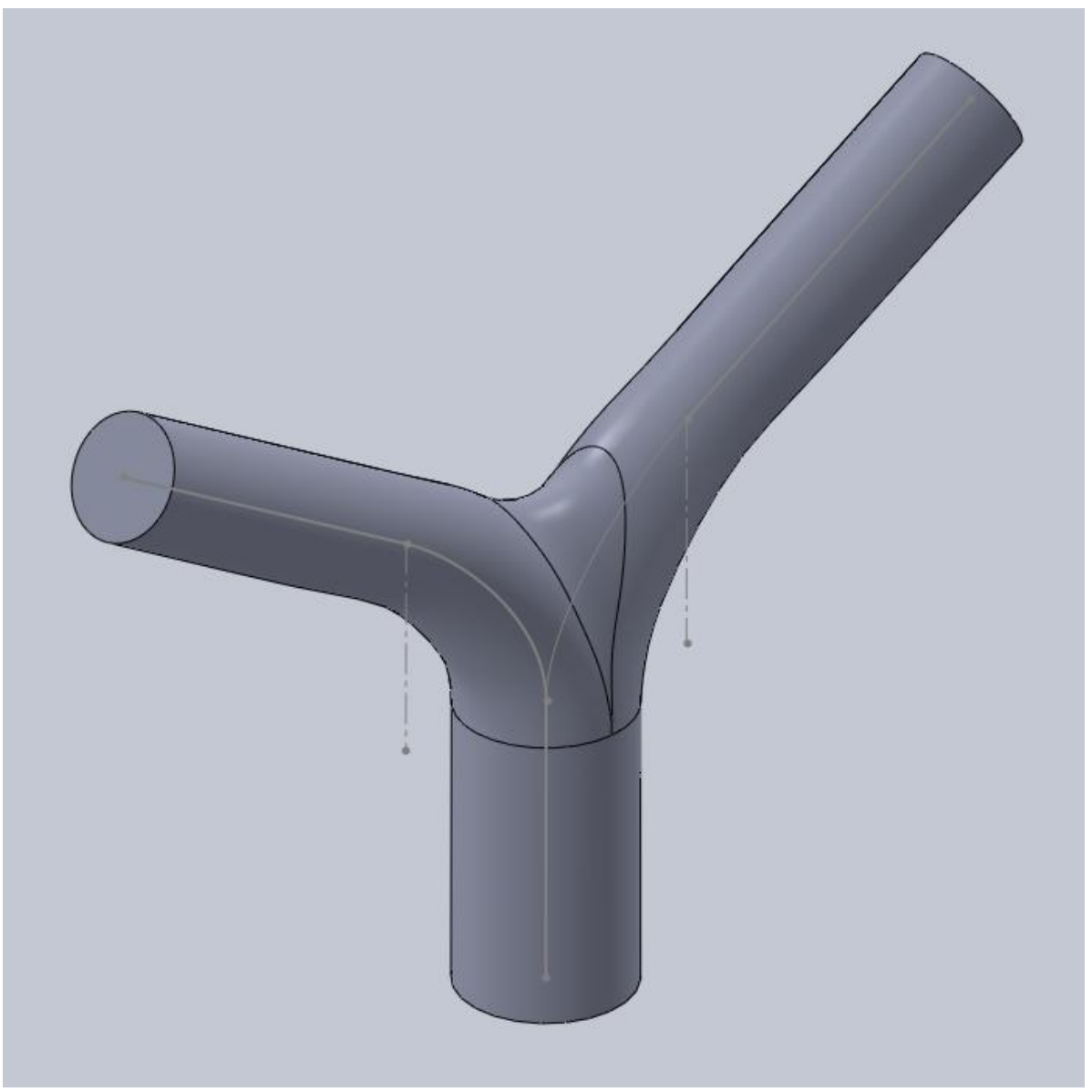

Figure 16: SolidWorks model of an idealized healthy MCA bifurcation. 


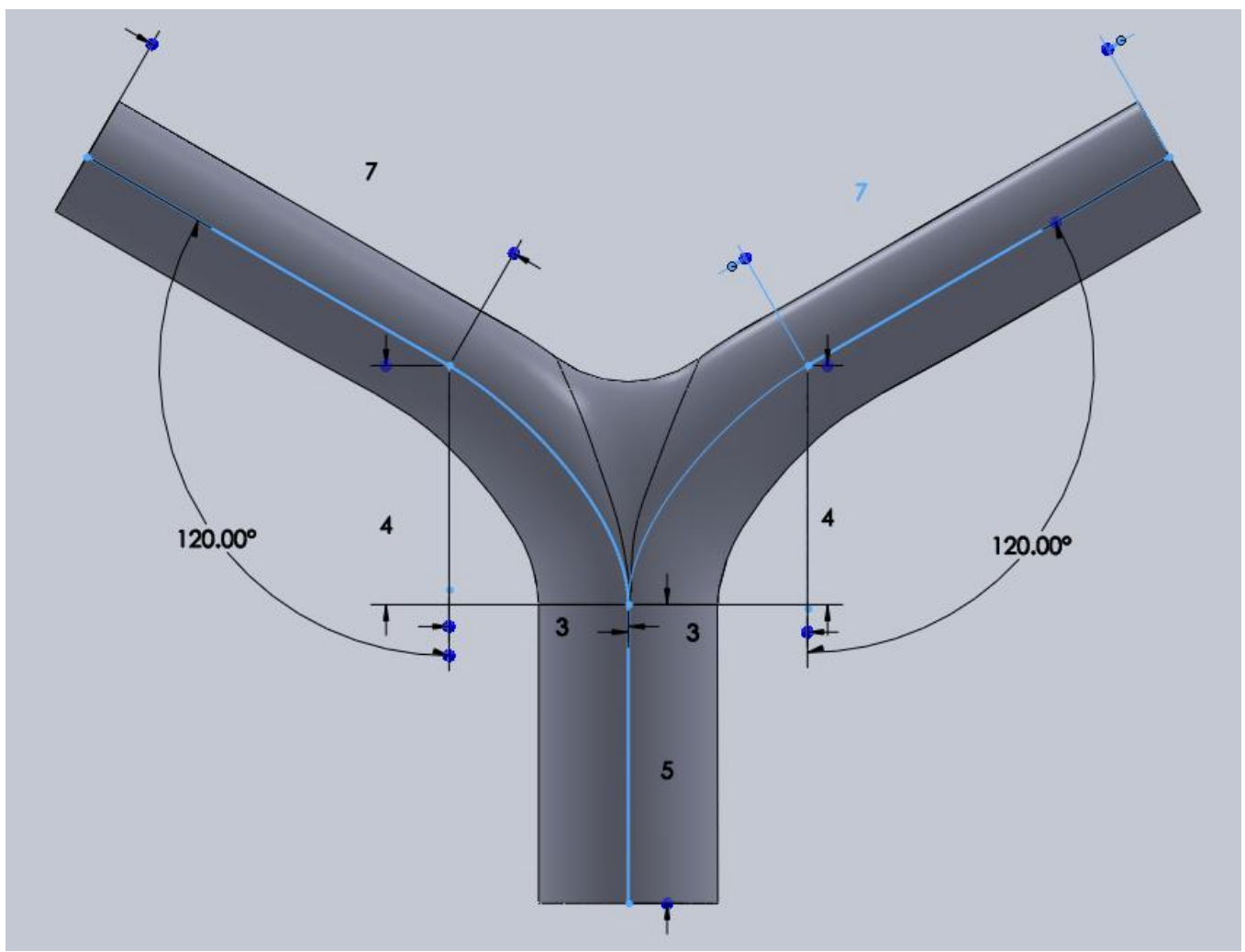

Figure 17: Dimensions for 120-120 bifurcation.

The LA ratio of 1 was chosen because it fell within the range for healthy MCA bifurcations, as well as the minimum LA ratio. A LA ratio of 1.6 was chosen because it was the cut off point for finding aneurysms at the MCA bifurcation. The final LA ratio, 2.2, was the average ratio for MCA bifurcations with aneurysms. The base angles were chosen based on bifurcation angles ranges for healthy and aneurysm diseased MCA bifurcations. 120 degrees was the average homeostatic angle. 90 degrees was the average angle for MCA bifurcations with aneurysms. 60 degrees was an extreme case found only in MCA bifurcations with aneurysms. The models were made to match images of the MCA bifurcation during operations or imaging since no digital scans were available to import into COMSOL. All models were imported into COMSOL and 
cleaned up using COMSOL CAD. To make computation faster, the models were split in half and a symmetry condition in laminar flow module was placed on the face of symmetry.

\section{4: Meshing}

Once all the models were imported and the simulations were ready, a mesh analysis was performed to find the minimum meshing required to get accurate results. The 120 base angle with a LA ratio of 1 model, the 120-120 model, was simulated for one cardiac cycle at various mesh settings until convergence was achieved. The average velocity of the inlet and outlets were recorded along with the shear stress at a point on the bifurcation at time 0.3 seconds. The results can be seen in Table I and Figure 18.

Table I: Convergence study of fluid velocities and shear stress for 120-120 model at 0.3 second

\begin{tabular}{r|cccc}
\hline DOF & $\begin{array}{c}\text { Inlet Velocity } \\
(\mathrm{m} / \mathrm{s})\end{array}$ & $\begin{array}{c}\text { Sup. Outlet } \\
\text { Velocity } \\
(\mathrm{m} / \mathrm{s})\end{array}$ & $\begin{array}{c}\text { Inf. Outlet } \\
\text { Velocity } \\
(\mathrm{m} / \mathrm{s})\end{array}$ & $\begin{array}{c}\text { Probed Shear } \\
\text { Stress } \\
(\mathrm{Pa})\end{array}$ \\
\hline 4060 & 0.48807 & 0.50112 & 0.49759 & 11.86948 \\
7228 & 0.5095 & 0.52037 & 0.52076 & 13.44398 \\
12676 & 0.5139 & 0.52352 & 0.52354 & 17.36827 \\
28772 & 0.52185 & 0.52639 & 0.52624 & 18.94956 \\
49928 & 0.51757 & 0.52079 & 0.52024 & 19.96198 \\
106912 & 0.52321 & 0.52483 & 0.52575 & 22.7529 \\
247900 & 0.52488 & 0.5235 & 0.52324 & 22.76307 \\
\hline \hline
\end{tabular}




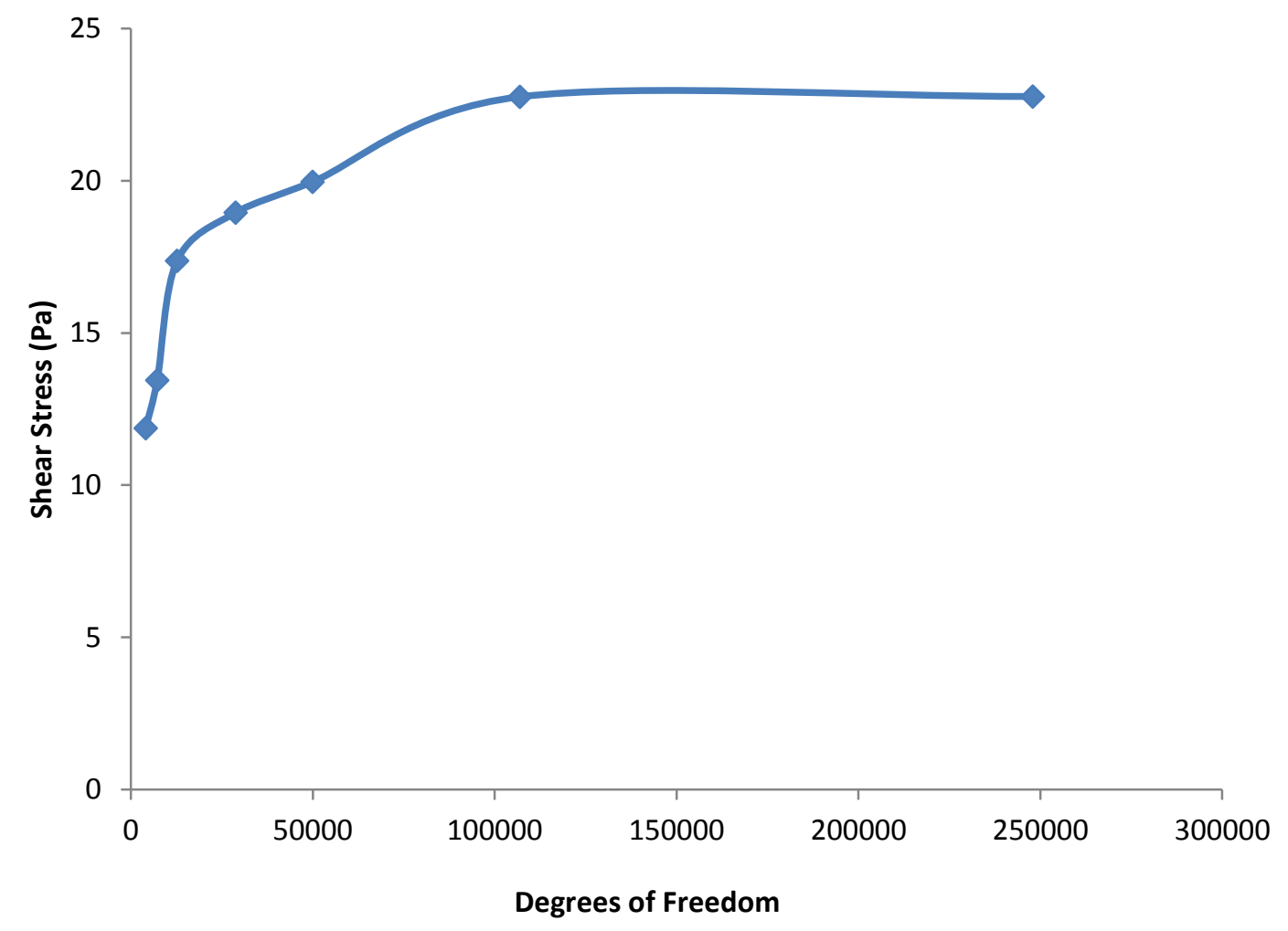

Figure 18: Line plot of probed shear stress at the bifurcation related to Degrees of freedom.

The shear stress and velocities both converged between 100k DOF and 250K DOF. The mesh settings used were COMSOL's predefined finer mesh size for fluid dynamics. Some minor alterations to the mesh settings were done to remove unneeded elements and nodes and increase element density around the apex of the MCA bifurcation. The fully meshed MCA bifurcation can be seen in Figure 19 below. 


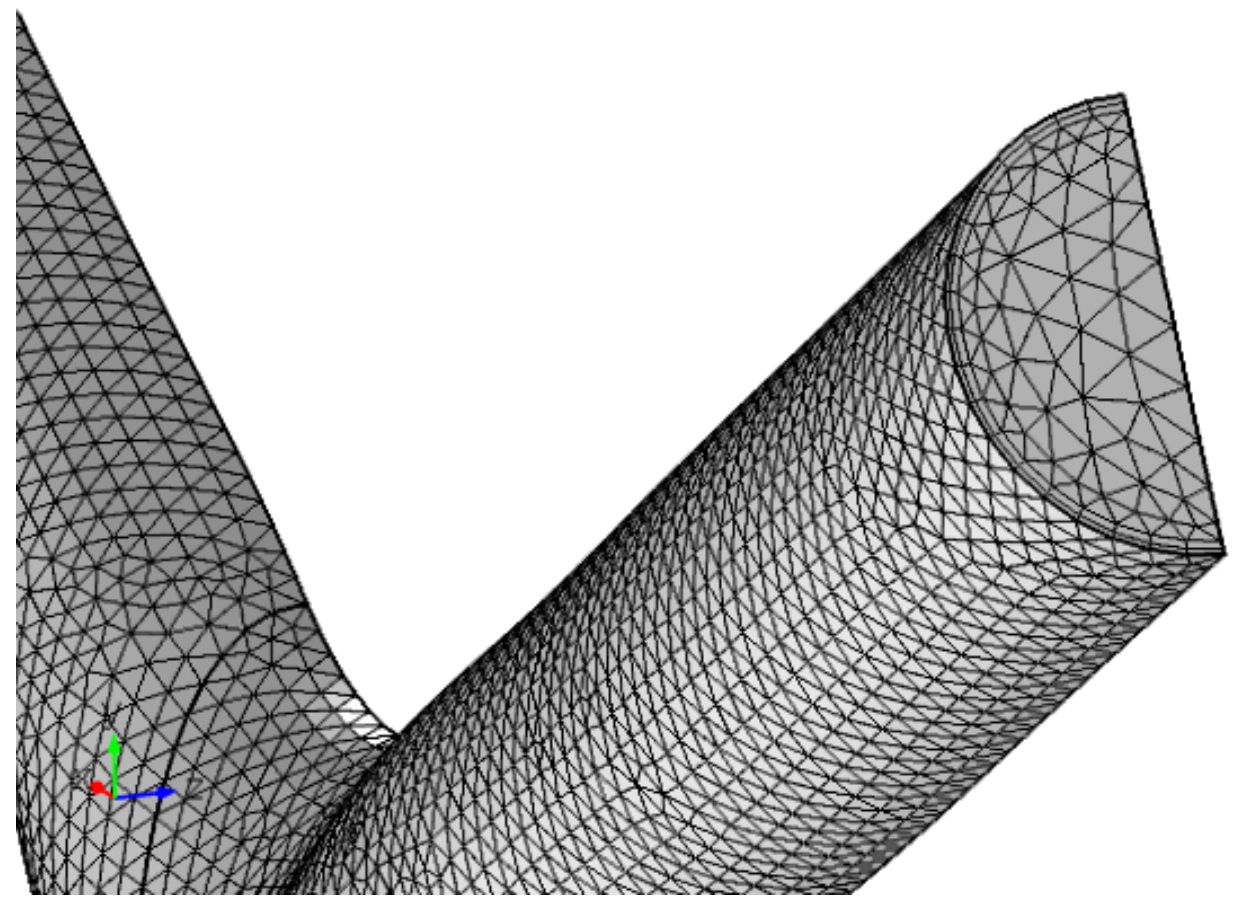

Figure 19: Mesh of the boundary and domain elements for $120^{\circ}-120^{\circ}$ model.

The domain was set to the predefined finer setting while the boundary was set to the predefined extra fine setting. The parent artery had boundary set to the predefined finer setting to reduce the number of elements on the parent vessel wall. The predefined settings used the mesh fluid dynamic setting in COMSOL. This setting created a gradient in element size with smaller elements being on the boundaries of the model and larger elements in the middle of the domain. Laminar flow causes little turbulence to the flow in the center of the vessel so less meshing is required to model the fluid. Meshing gets finer closer to the boundary because the fluid velocity reduces rapidly as it approaches a velocity of 0 , the no slip condition. The predefined mesh fluid dynamic settings also added a layer of elements between the boundary and the domain. These elements are extremely thin and help approximate the wall shear stress more accurately. Two were 
used since additional layers had little effect. The final settings can be seen in Figure 19 above with a domain mesh size range of $0.318 \mathrm{~mm}$ to $0.095 \mathrm{~mm}$ and a boundary mesh size range of $0.178 \mathrm{~mm}$ to $0.019 \mathrm{~mm}$. These sizes match other computational fluid dynamic models and should accurately model all the MCA bifurcation models [7]. All models used a mixture of hexahedral and tetrahedral elements. Hexahedral elements approximate stresses better so they were used for the boundaries of the model where wall shear stress will be calculated. Tetrahedral elements were used for the domain since they model complex 3D structures more accurately. All models were run on the BMED local server cluster with a computation time ranging from 1 to 2 hours depending on the number of elements and study length.

\section{5: PIV Settings}

A particle tracing module was added when all the settings were finalized and the fluid velocities were simulated. The particle study would be compared to a PIV study by Shigekazu Takeuchi and Taeshi Karino as another verification method for the computer model [47]. The PIV study was performed on ICAs and MCAs from human cadavers at normal flow rates. The 120-120 model was used since it was the closest match to the geometries of the cadaver's MCA. Fluid particle tracing works in COMSOL by using pre-simulated fluid flow and applying particle that interact with the fluid flow. The loading conditions for the particle study were set to $3.1 \mathrm{ml} / \mathrm{s}$ in order to match the PIV experiment. This was achieved by putting a pressure drop of $447 \mathrm{~Pa}$ between the inlet and outlets. Particles with a density of $1060 \mathrm{~kg} / \mathrm{m}^{3}$ and with a diameter of $80 \mu \mathrm{m}$ were created to match blood and the particles use in the PIV study. 125 particles were set to be released from the inlet every 0.01 seconds matching the fluid velocity at the inlet. To 
simulate how the particles would react to the fluid, a drag force was applied to each particle. The drag force used the simulated fluid velocity and the viscosity to apply a force to the particle using equation 16 and 17.

$$
\begin{gathered}
F=\frac{1}{\tau_{p}} m_{p}(u-v) \\
\tau_{p}=\frac{\rho_{p} d_{p}}{18 \mu}
\end{gathered}
$$

$m_{p}$ is the mass of the particle, $\rho_{p}$ is the density of the particle, $d_{p}$ is the diameter of the particle, $\mu$ is the viscosity of the fluid, $\mathrm{u}$ is the velocity of the fluid, and $\mathrm{v}$ is the particle velocity. This force causes the particle to change its velocity with regards to the changing fluid flow and viscosity. The model walls and symmetry plane were set to bounce the particles off with the outlets removing the particles. Bounce was chosen because the particles would bounce off the walls in the PIV. 


\section{CHAPTER 4: RESULTS}

\section{1: Initial Testing}

A preliminary model of a simple cylinder was simulated to compare COMSOL's fluid physics to proven analytical equations. The cylinder was $3 \mathrm{~mm}$ in diameter and $10 \mathrm{~mm}$ long. A pressure drop of $747 \mathrm{~Pa}$ was placed between the inlet and outlet to create an average velocity of $0.6 \mathrm{~m} / \mathrm{s}$. A stationary study was performed using the same mesh settings as the MCA models, leading to a final mesh of 60,000 tetrahedral and hexahedral elements. The analytical equations used were equation 1, Hagen-Poiseulle, and equation 10, wall shear stress for a straight tube. The average velocity and average wall shear stress were then simulated for blood as a Newtonian and non-Newtonian fluid in the cylinder. The results can be seen in Table II.

Table II: Comparison between analytical equations and simulation results for simple cylinder.

\begin{tabular}{lcc|cc}
\hline & \multicolumn{2}{c}{ Velocity [m/s] } & \multicolumn{2}{c}{ Shear stress [Pa] } \\
& Newtonian & Non-Newtonian & Newtonian & Non-Newtonian \\
\hline Hand Calculation & 0.6 & 0.5645 & 5.6 & 5.952 \\
Simulation & 0.59518 & 0.5560 & 5.54672 & 5.920 \\
\hline Error [\%] & 0.8033 & 1.5058 & 0.9514 & 0.5376 \\
\hline \hline
\end{tabular}

The analytical equations were quite accurate when compared to the computer simulation. Very little error, less than $1 \%$, was observed between the Newtonian models and the hand calculations. The non-Newtonian model had higher error in the velocity compared to the shear stress, but was still very low. The low error indicated that the fluid physics were correctly implemented in the computer model and accurately simulated blood flow through a straight cylinder. 


\section{2: Model Validation}

To ensure that the loading conditions and geometries were also implemented correctly, a comparison between literature values and the healthy $120^{\circ}-120^{\circ} \mathrm{MCA}$ bifurcation was performed. The model was simulated over two cardiac cycles with nonNewtonian laminar flow. The minimum, maximum, and average fluid flow rate and velocity were calculated at the inlet. The average wall shear stress on the vessel wall and the max wall shear stress at the bifurcation were also calculated. The data comparing the simulation to literature is tabulated in Table III.

Table III: Comparison of literature values to MCA control model

\begin{tabular}{lcc}
\hline & Literature & Simulation \\
\hline Velocity range $[\mathrm{m} / \mathrm{s}]$ & $0.23-1.06$ & $0.241-0.539$ \\
Average Velocity $[\mathrm{m} / \mathrm{s}]$ & $0.32-0.82$ & 0.38 \\
Flow rate range $[\mathrm{ml} / \mathrm{s}]$ & $1.7-6.0$ & $1.71-3.81$ \\
Average flow rate $[\mathrm{ml} / \mathrm{s}]$ & $2.5-3.3$ & 2.71 \\
Shear stress on vessel wall $[\mathrm{Pa}]$ & $1.0-7.0$ & 3.93 \\
Max Shear Stress $[\mathrm{Pa}]$ & $25-35$ & 41.31 \\
\hline \hline
\end{tabular}

The velocity of blood in the simulated model was lower when compared to the velocity range found in literature. The velocity averaged over the cardiac cycle was particularly low. The velocity is quite dependent on the radius of the blood vessel so it was expected that the simulation would have velocities on the low side because a large vessel radius was used. The flow rate is a better indicator for model accuracy it is determined by the nutrient requirements of the brain and vary less between people. The flow rate range and average flow rate were accurate and fell within the range of literature values. The vessel wall shear stress fell within the homeostasis range for arteries, but the 
max shear stress was higher than literature values. The max shear stress was found just after the bifurcation. The max shear stress was high compared to the limited studies analyzing the shear stress at the MCA bifurcation. Additionally, wall shear stress greater than $40 \mathrm{~Pa}$ is common in the Circle of Willis [7]. The control model was within or close to healthy blood flow and shear stress values, indicating the geometries and fluid flow modules were correct.

Additionally, the control $120^{\circ}-120^{\circ}$ bifurcation model was simulated with the COMSOL particle tracing module and compared to a journal article that performed PIV on MCAs taken from cadavers. The PIV experiment used a steady flow rate with a mixture of different sized plastic beads in a blood like solution. The control model was set up to have similar loading conditions as the journal article and was seeded so that a similar trace density was achieved. Figure 20 compares the PIV from the article to the particle tracings from the COMSOL model.

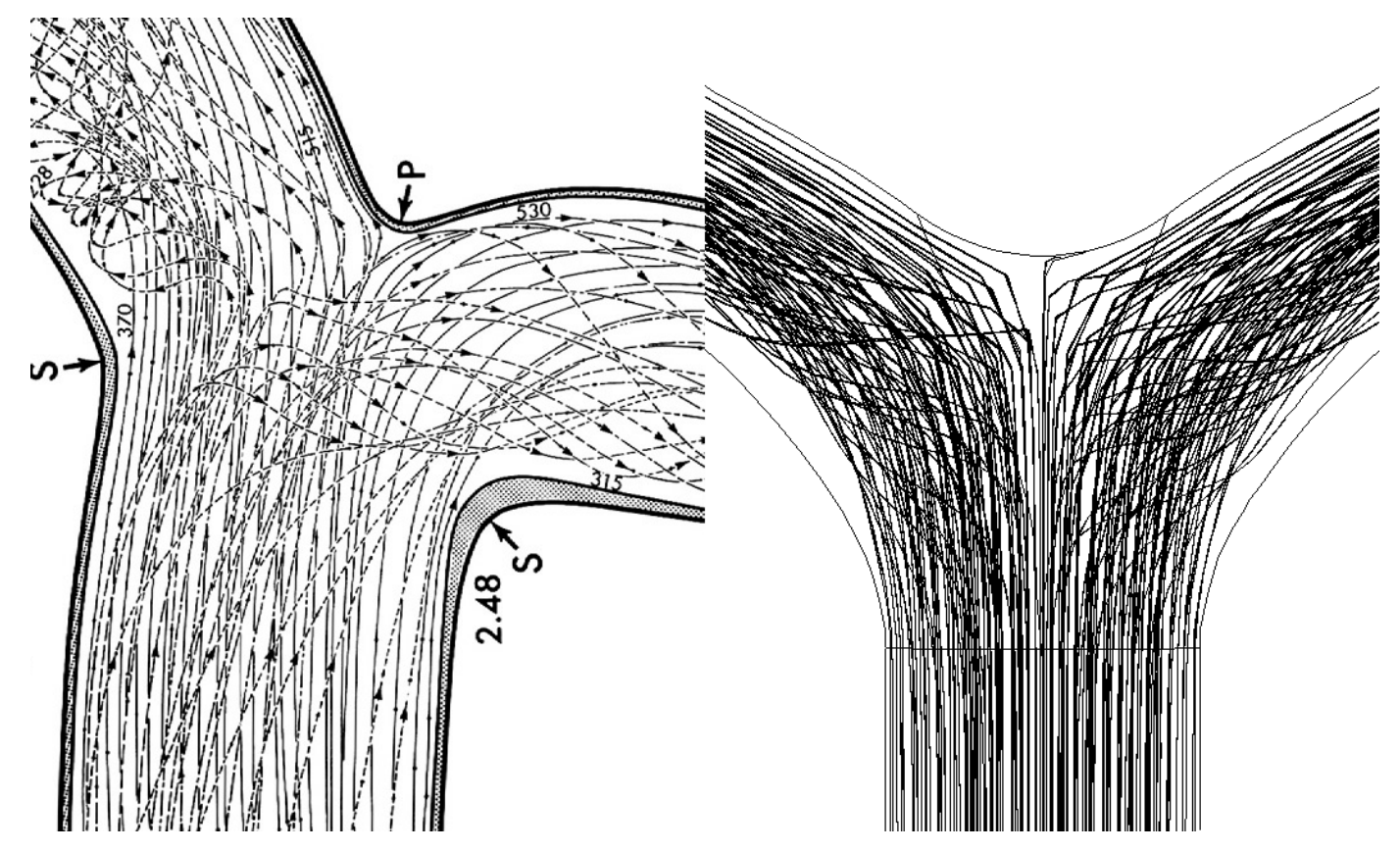

Figure 20: (left)PIV experiment from literature. (right)Particle tracings from simulated model. 
The computer particle trace looks similar to the PIV experiment. Both have relatively straight flow lines in the parent artery with an equal split at the bifurcation. No backflow is present in both. The main difference between the PIV study and the computer model was the decrease in vortices in the computer model. The computer model had the vortices at the bifurcation, but did not develop secondary vortices after the split. This could be attributed to the perfectly smooth vessel walls in the model, the model only showing half of the vortices due to symmetry conditions, or a perfectly linear flow rate into the bifurcation. The equal split and the initial vortices indicate that the computer particle trace is satisfactory and further supports that the simulation is working as intended.

\section{3: Fluid Flow}

A comparison of fluid flow profiles were simulated using the laminar flow module in COMSOL for every angle iteration of the MCA bifurcation. All models were simulated for two cardiac cycles, 1.616 seconds, using $0.008 \mathrm{sec}$ time steps. Loading conditions and fluid settings were the same between models with the only change being the bifurcation angles. Figure 21 shows the comparison between the flow profiles at peak systole for all models. Enlarged images of each individual flow profile can be found in appendix B. 

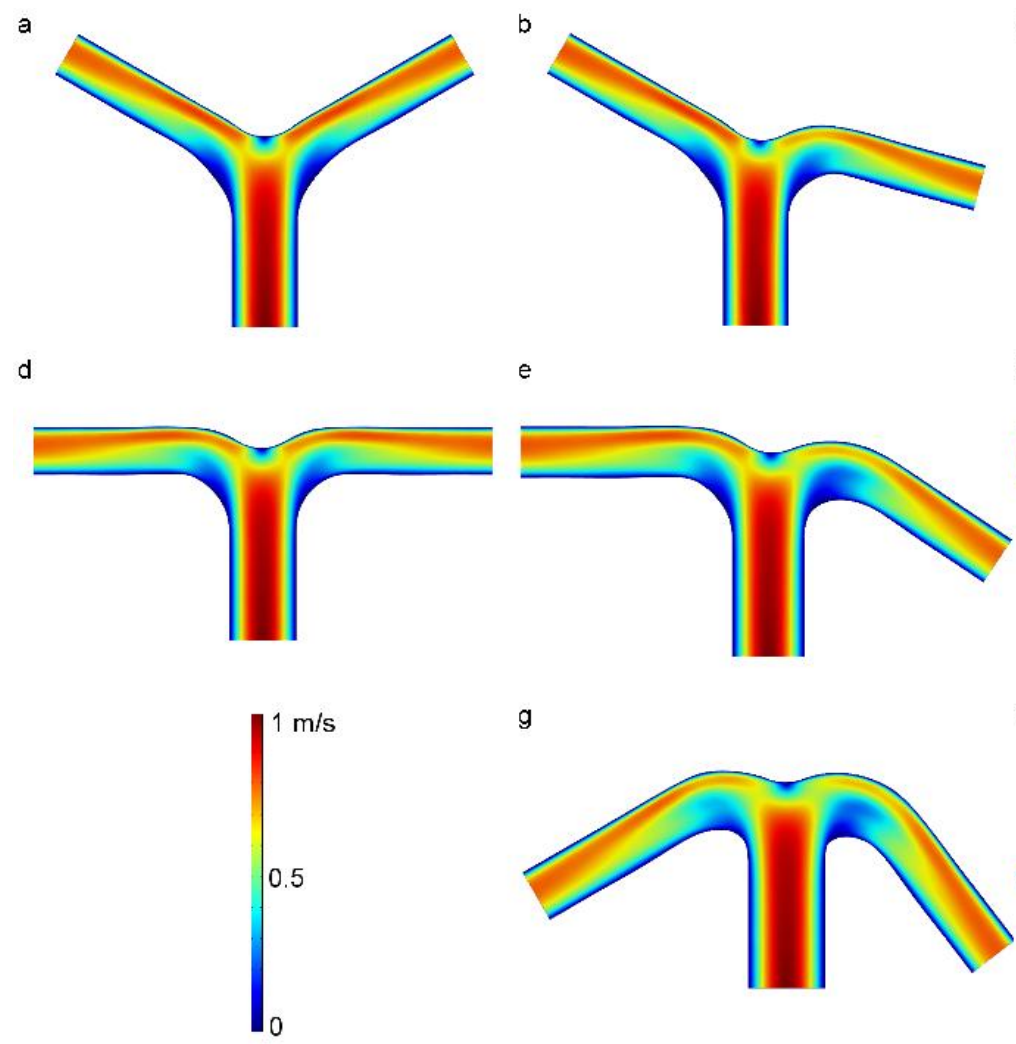

e

g

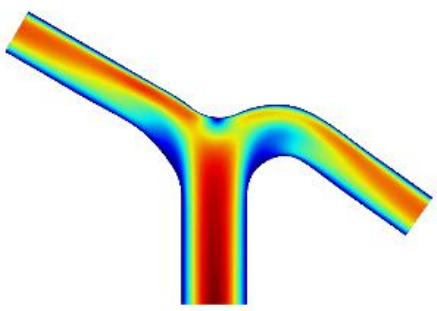

f

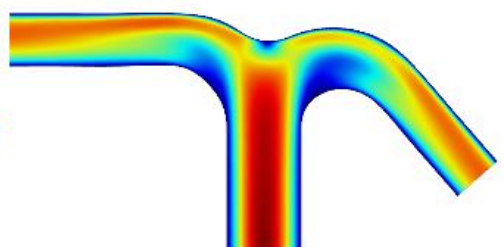

h

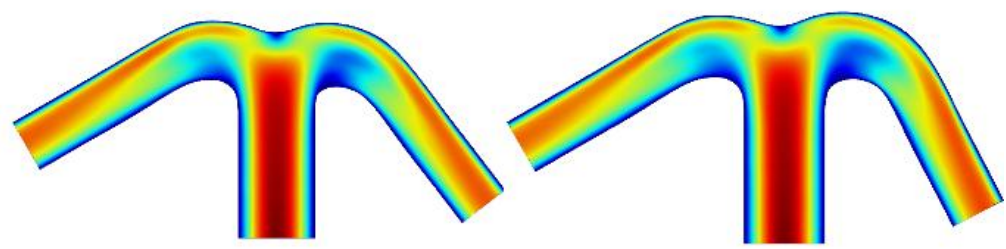

Figure 21: Flow profile at peak systole for all bifurcation angles. (a-h) $120^{\circ}-120^{\circ}, 120^{\circ}$ $75^{\circ}, 120^{\circ}-54.54^{\circ}, 90^{\circ}-90^{\circ}, 90^{\circ}-56.25^{\circ}, 90^{\circ}-40.91^{\circ}, 60^{\circ}-37.5^{\circ}, 60^{\circ}-27-27^{\circ}$ respectively

The peak velocity for every model was around $1 \mathrm{~m} / \mathrm{s}$. All models had little to no flow at the tip of the bifurcation and interior side of the bifurcation curves. Increasing the LA ratio caused the area of slow flow on the interior side to increase in size. The slow flow at the bifurcation tip was not affected by changing the bifurcation angle or the LA ratio. The inlet profile and outlet profile for both daughter arteries look similar for all models. The side with the smaller bifurcation angle appeared to have slower velocities during the bifurcation, but matched once the vessel straightened.

Post processing was used to average the flow rates at the inlet and outlets for one cardiac cycle. The flow rates was averaged over the 2 nd cardiac cycle, 0.808 to 1.616 
seconds, to avoid undeveloped flow profiles caused by zero fluid flow in the model at time zero. All models had the same length from the inlet to the outlets. The flow rates for each model can be found in Table IV.

Table IV: Table of averaged inlet and outlet flow rates for all models.

\begin{tabular}{l|ccc}
\hline \multicolumn{1}{l}{} & $\begin{array}{c}\text { Inlet flow rate } \\
{[\mathrm{ml} / \mathrm{s}]}\end{array}$ & $\begin{array}{c}\text { Superior outlet } \\
\text { flow rate [ml/s] }\end{array}$ & $\begin{array}{c}\text { Inferior outlet } \\
\text { flow rate } \\
{[\mathrm{ml} / \mathrm{s}]}\end{array}$ \\
\hline $120-120$ & 2.721 & 1.363 & 1.366 \\
$120-75$ & 2.693 & 1.374 & 1.326 \\
$120-54.54$ & 2.692 & 1.369 & 1.329 \\
$90-90$ & 2.680 & 1.345 & 1.341 \\
$90-56.25$ & 2.679 & 1.350 & 1.335 \\
$90-40.9$ & 2.698 & 1.351 & 1.343 \\
$60-37.5$ & 2.591 & 1.303 & 1.286 \\
$60-27.27$ & 2.608 & 1.326 & 1.279 \\
\hline \hline
\end{tabular}

The flow rate splits in half for both $120^{\circ}-120^{\circ}$ and $90^{\circ}-90^{\circ}$ models. Increasing the LA ratio caused the flow rate not to divide equally. The flow rate was higher in the side with the greater bifurcation angle. Also, the inlet flow rate decreased as the base bifurcation angles become smaller. Increasing the LA ratio also lead to smaller decrease in inlet flow rates. The decrease in inlet flow rate lead to a decrease in outlet flow rates. Overall, very little change between flow rates was found.

\section{4: Wall Shear Stress}

Additionally, wall shear stress was compared between models. The wall shear stress was calculated using the viscosity and shear rate. The wall shear stress was 
calculated over two cardiac cycles. The results of wall shear stress at peak systole can be seen below in Figure 22 with enlarge images of each models shear stress in appendix B.
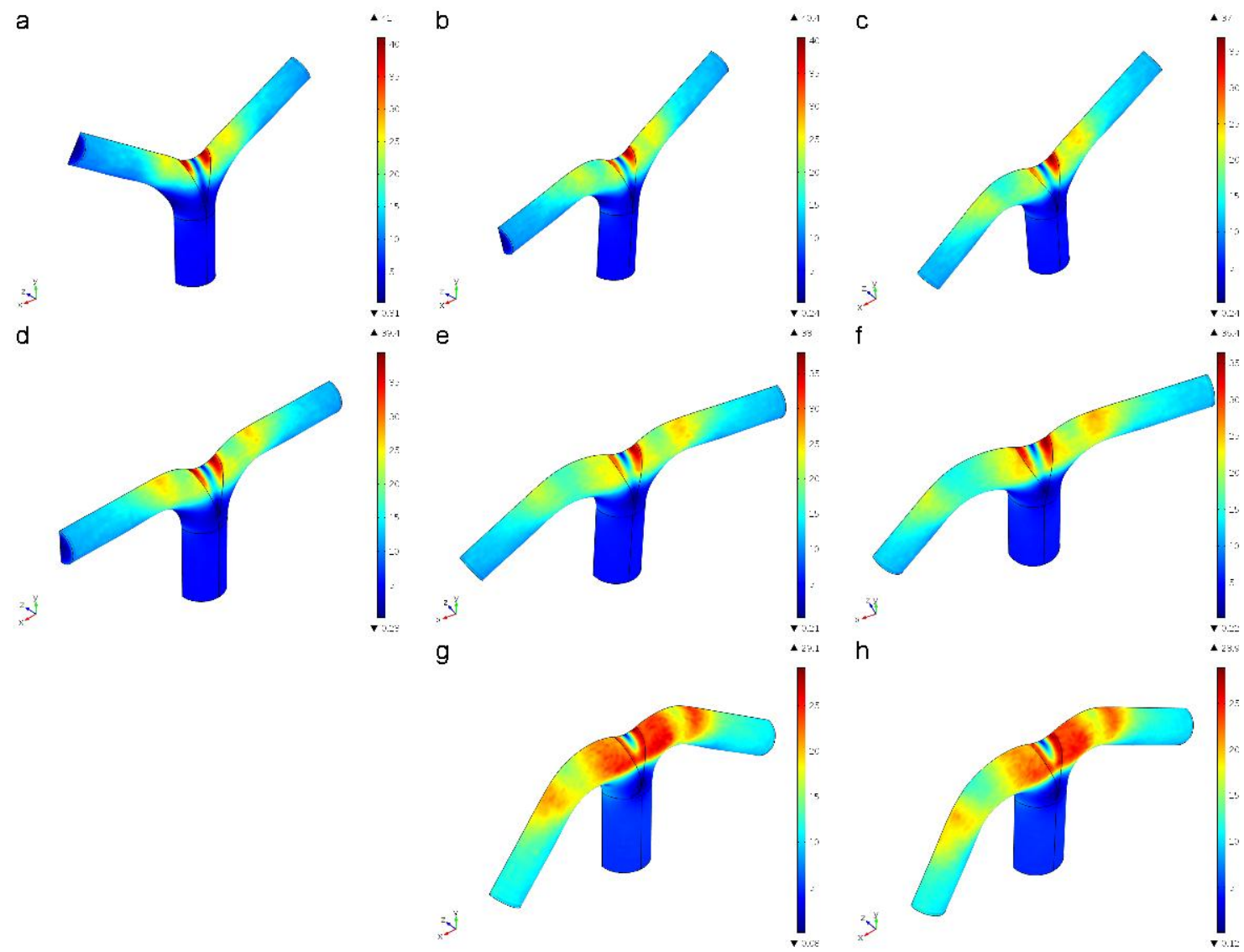

Figure 22: Shear Stress Comparison. (a-h) $120^{\circ}-120^{\circ}, 120^{\circ}-75^{\circ}, 120^{\circ}-54.54^{\circ}, 90^{\circ}-90^{\circ}$, $90^{\circ}-56.25^{\circ}, 90^{\circ}-40.91^{\circ}, 60^{\circ}-37.5^{\circ}, 60^{\circ}-27-27^{\circ}$ respectively.

For all models, two points of maximum shear stress appeared just after the bifurcation on both daughter arteries. The max shear was always on the opposite side of where the parent artery entered the bifurcation. Changes to the base bifurcation angle and the LA ratio caused the shear stress to decrease in magnitude and spread out through the daughter arteries. The base angles, $120^{\circ}, 90^{\circ}, 60^{\circ}$, were quite different compared to 
each other, while the LA ratio changes had little effect on wall shear stress. The $120^{\circ}$ base angle had most of its wall shear stress localized right after the bifurcation. All LA ratios had a distinct separation between the shear stress on the superior M2 and the inferior M2 arteries. Increasing the LA ratio only caused a slight drop in max shear stress, $41 \mathrm{~Pa}$ to $39 \mathrm{~Pa}$. The $90^{\circ}$ base angle models had less separation of shear stress with the point of bifurcation being surrounded with $15 \mathrm{~Pa}$ of shear stress. Increasing the LA ratios caused an increase in unification of the shear stress at the bifurcation. The drop in max shear stress as the LA ratio increased was $39 \mathrm{~Pa}$ to $36 \mathrm{~Pa}$. The $60^{\circ}$ base angle models had complete unification of the shear stress between the branched arteries. The area of unification also had increased shear stress, around $25 \mathrm{~Pa}$. The peak shear stress was much lower, around $29 \mathrm{~Pa}$ for both models, and was not affected by the LA ratio.

To quantify the changes in shear stress between models, the max wall shear stress during systole and diastole were calculated from every MCA simulation. A 16 Pa cutoff was used to determine the area of high shear stress at the bifurcation. This was done by using COMSOLs post processing boundary integration function using equation 18 as the argument.

$$
16[P a]>s p f . m u * s p f . s r
$$

spf.mu is the viscosity and spf.sr is the shear rate calculated by the simulation. 16 $\mathrm{Pa}$ was used because it is the average shear stress at the MCA bifurcation. The percent change was calculated using to the healthy $120^{\circ}-120^{\circ} \mathrm{MCA}$ model as the control. The shear stress found in each model can be seen in Table V and Table VI. 
Table V: Max shear stress during systole and diastole with maximum area of shear stress at MCA bifurcation

\begin{tabular}{|c|c|c|c|}
\hline & \multicolumn{2}{|c|}{ Max Shear (Pa) } & \multirow{2}{*}{$\begin{array}{l}\text { Area of Shear } \\
\text { Stress }\left(\mathrm{mm}^{\wedge} 2\right)\end{array}$} \\
\hline & Systole & Diastole & \\
\hline $120-120$ & 41.31 & 12.33 & 19.41 \\
\hline $120-75$ & 40.78 & 11.88 & 22.77 \\
\hline $120-54.54$ & 39.71 & 11.91 & 24.52 \\
\hline $90-90$ & 39.57 & 11.22 & 24.11 \\
\hline $90-56.25$ & 38.28 & 11.08 & 26.87 \\
\hline $90-40.9$ & 36.57 & 10.76 & 25.37 \\
\hline $60-37.5$ & 29.18 & 8.71 & 27.96 \\
\hline $60-27.27$ & 28.90 & 9.10 & 28.39 \\
\hline
\end{tabular}

Table VI: Percent change in shear stress between different models compared to 120-120 control model

\begin{tabular}{l|ccc}
\hline \multicolumn{1}{l}{} & Systole & Diastole & $\begin{array}{c}\text { Area of shear } \\
\text { stress }\end{array}$ \\
\hline $120-120$ & $0.00 \%$ & $0.00 \%$ & $0.00 \%$ \\
$120-75$ & $1.28 \%$ & $3.65 \%$ & $17.34 \%$ \\
$120-54.54$ & $3.87 \%$ & $3.40 \%$ & $26.35 \%$ \\
$90-90$ & $4.21 \%$ & $9.01 \%$ & $24.26 \%$ \\
$90-56.25$ & $7.33 \%$ & $10.16 \%$ & $38.46 \%$ \\
$90-40.9$ & $11.47 \%$ & $12.72 \%$ & $30.71 \%$ \\
$60-37.5$ & $29.36 \%$ & $29.39 \%$ & $44.10 \%$ \\
$60-27.27$ & $30.04 \%$ & $26.19 \%$ & $46.31 \%$ \\
\hline \hline
\end{tabular}

Comparing the models, it appears that there was a decrease in maximum shear stress in both systole and diastole as the angle of bifurcation becomes more asymmetrical and as the base angle becomes smaller. Only a 3.87\% change was observed when increasing the LA ratio for the $120^{\circ}$ models. The $90^{\circ}$ models had a larger change in shear stress with LA ratio increase. The shear stress decreased by $7.26 \%$ when comparing the $90^{\circ}$ model with LA ratio of 1 to LA ratio of 2.2. Diastole showed similar drops in wall 
shear stress. The area of the bifurcation affected by high shear stress increased when the LA ratio increased and when the bifurcation angle decreased.

Next, the wall shear stress was plotted versus the distance from the bifurcation point. A 2D plot of wall shear stress was taken from the edge created by the plane of symmetry intersecting the top of the MCA bifurcation. Wall shear stress was calculate using the viscosity and shear rate at the boundary of the model. The data was moved into excel and the center of the bifurcation was set as the origin. The $60^{\circ}$ base angle was compared to the control model. The remaining models were compared within their respective base angle. The wall shear stress to distance from bifurcation can be seen in Figure 23, Figure 24, and Figure 25.

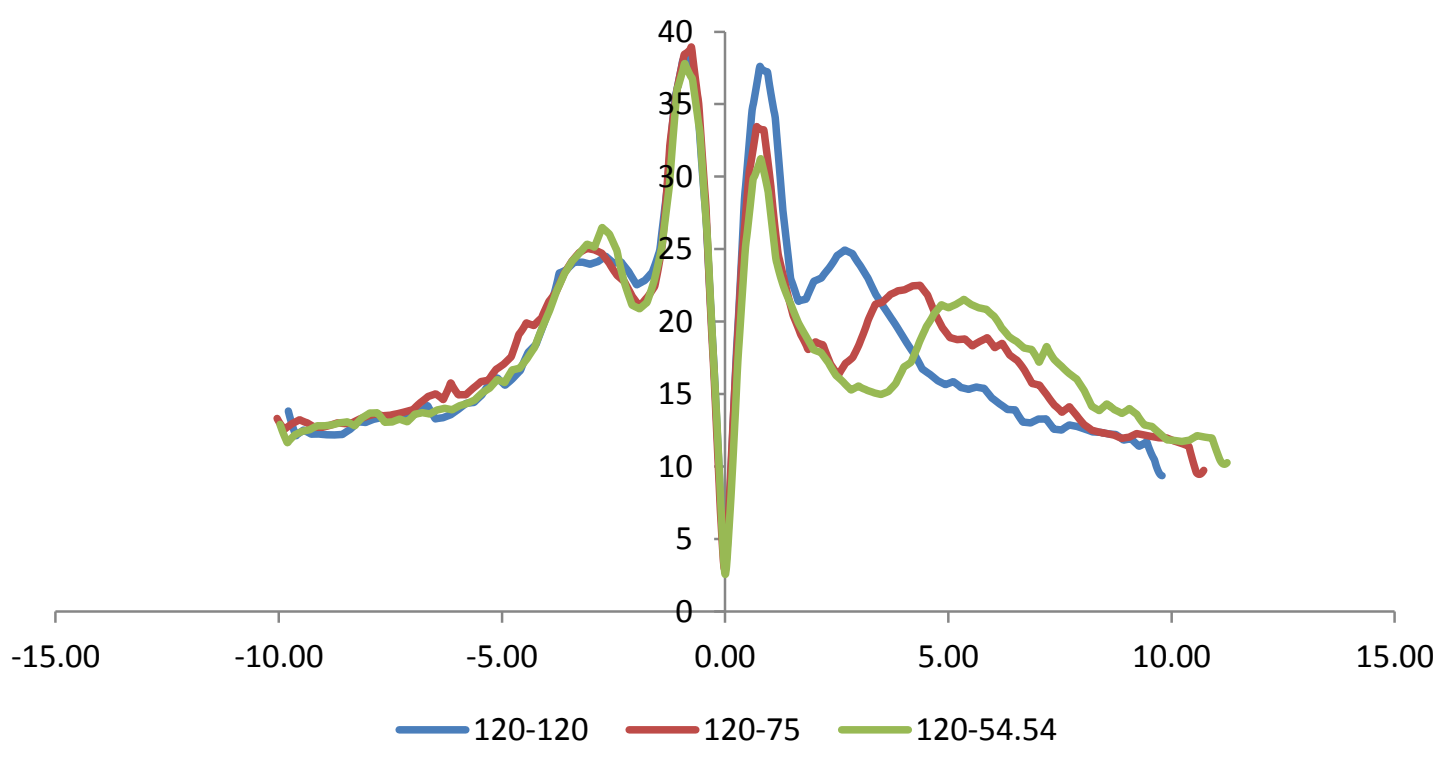

Figure 23: Shear stress versus Distance from bifurcation for $120^{\circ}$ base angle. 


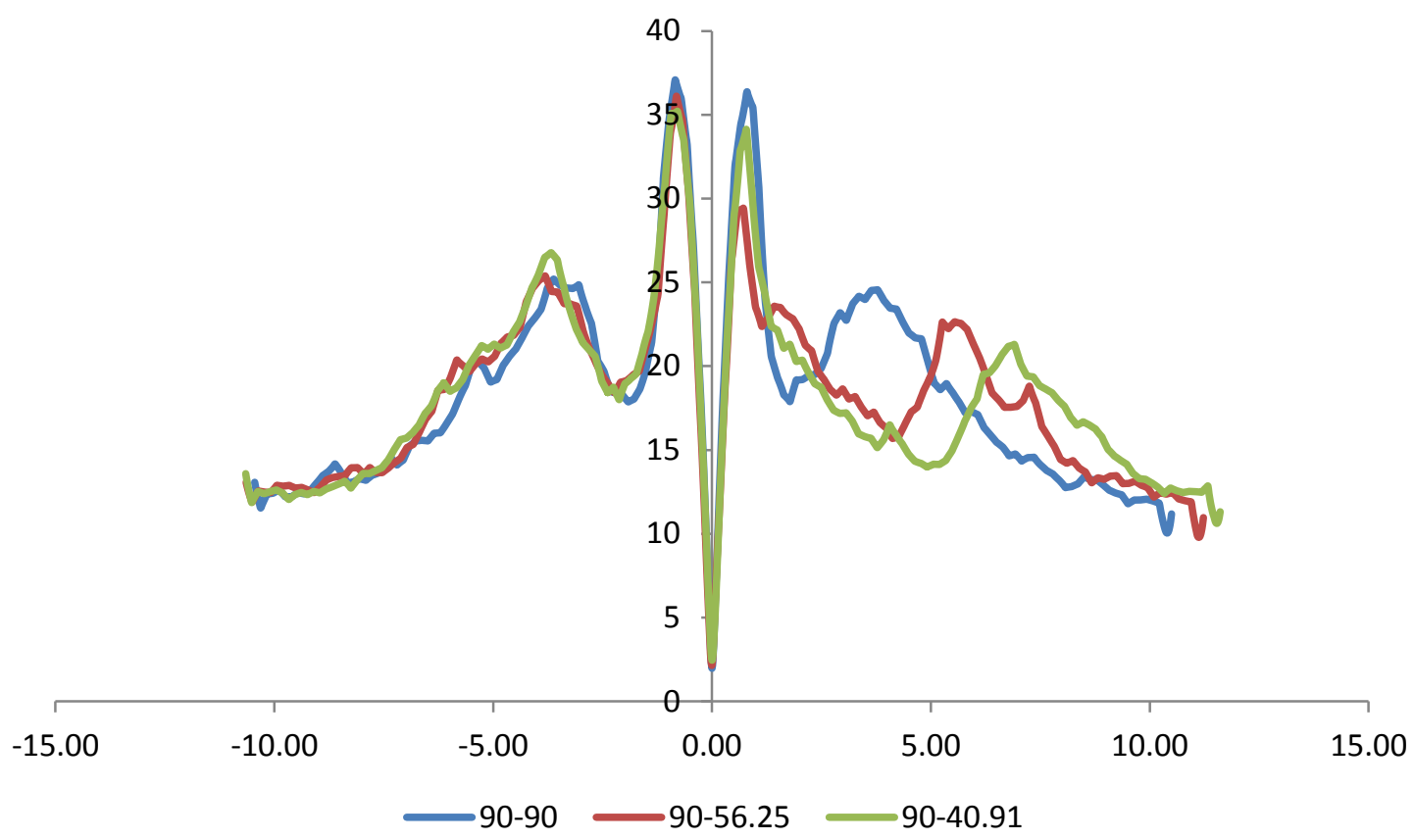

Figure 24: Shear stress versus Distance from bifurcation for $90^{\circ}$ base angle.

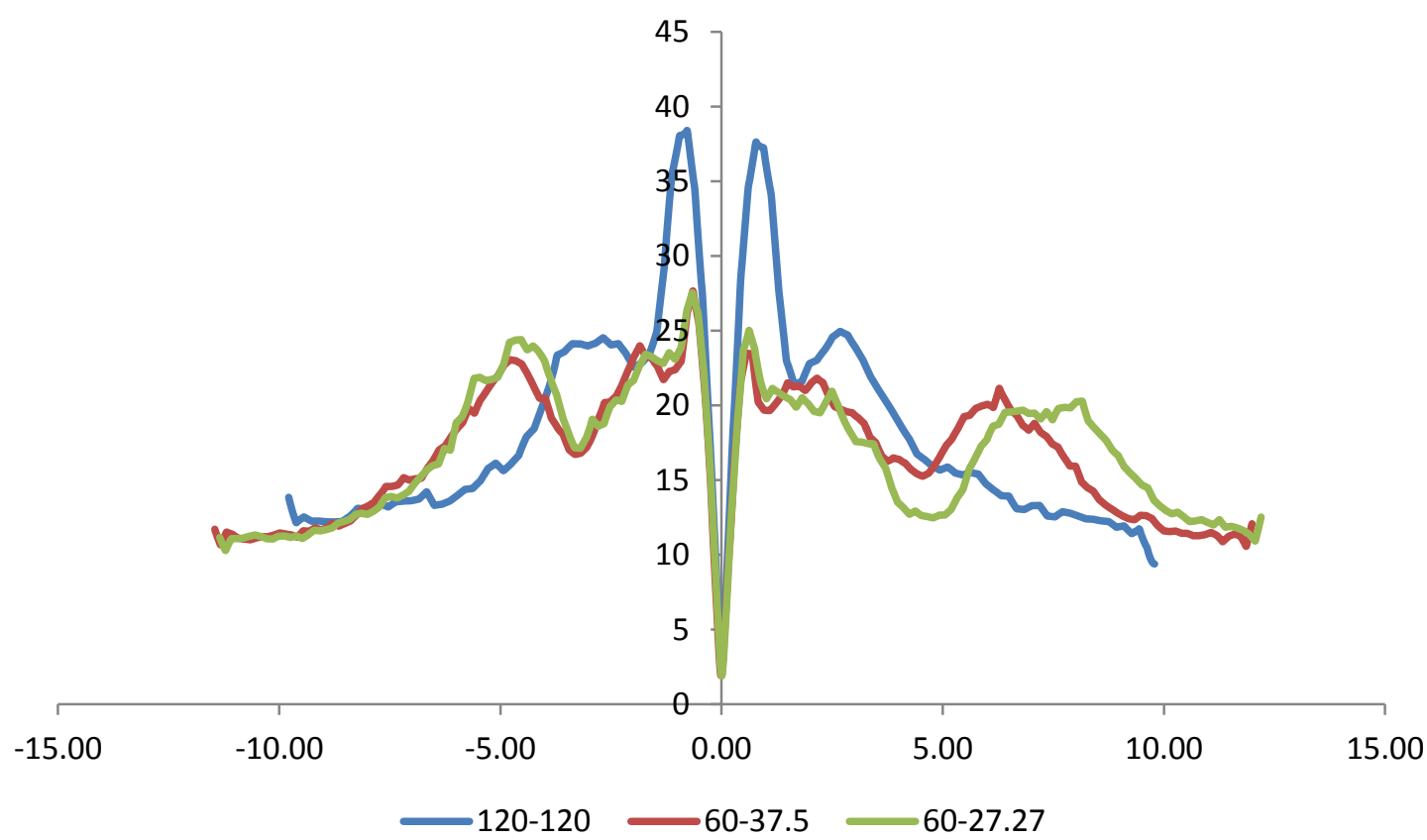

Figure 25: Shear stress versus Distance from bifurcation for $60^{\circ}$ base angle. 
Normal MCA bifurcations have two peaks of wall shear stress. The first peak appears right after the bifurcation and then a second, smaller, peak occurs further down the vessel. The second peak moves further away from the point of bifurcation as the bifurcation angle decreases, either from LA ratios or smaller base angle. The distance shifted by the second shear stress peak seemed equal when comparing angle decreases caused by LA ratios or the base angle. Little change is observed on the base angle side of the bifurcation when the LA ratio changes.

Finally, shear stress was plotted over an entire cardiac cycle for all models. A probe point was placed at the point of max shear stress and the shear stress was calculated using the boundary viscosity and shear rate. Again, the second cardiac cycle was used to avoid initial flow anomalies when loading conditions are first applied. All models were plotted against each other to create Figure 26. 


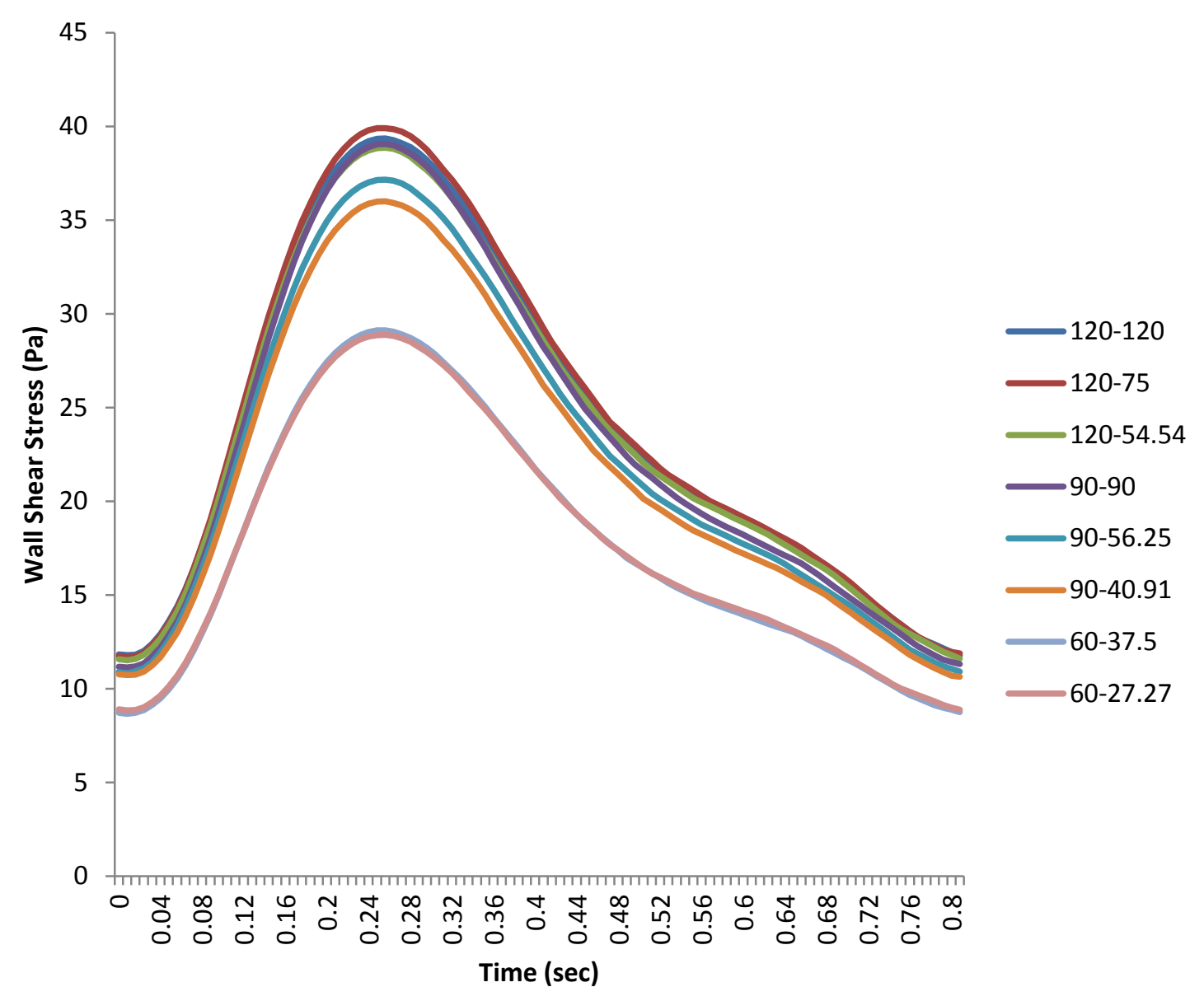

Figure 26: Probed max shear stress of all models over one cardiac cycle

Changing the LA ratios for the $120^{\circ}$ base models caused no noticeable changes over the cardiac cycle. The $90^{\circ}-90^{\circ}$ model had similar shear stress as the $120^{\circ}$ angle models, but increasing the LA ratio caused a notable decrease in peak shear stress during systole. A slight drop in diastole occurred also, but the overall waveform was similar to the $120^{\circ}$ models. The $60^{\circ}$ base models had matching waveforms with low shear stress in systole and diastole. The waveforms between all probed models look the same, just difference magnitudes in the peak and the start/end point. 


\section{CHAPTER 5: DISCUSSION}

Overall, the flow rate and profile changed little with changes to the bifurcation angle. It was expected that the outlet flow rate would be affected by the angle of bifurcation. Fluids prefer the path of least resistance so the more linear path, the lower the resistance. This led to a slight difference in flow rate between the daughter arteries with the smaller bifurcation angle having a lower flow rate. Additionally, decreases in the base bifurcation angles caused the overall flow rate in and out of the model to drop. This is probably due to the added resistance to flow caused by the geometries caused by head loss, fluid energy lost due to friction, as the fluid changes direction. Greater vortices were observed with larger LA ratio, but aneurysms tend to not form on the inside of curves. These vortices though leave less room for fluid to flow into the daughter artery and helps increase flow rate around the top portion of the bifurcation causing higher wall shear stress across more area.

The base angle and LA ratios had a much greater impact on shear stress compared to the fluid flow profiles. Decreasing the base angle caused greater bending leading to additional head loss which was converted into shear stress. Changing the LA ratio had the same, but smaller effect on shear stress. Surprisingly, the change in shear stress with changes to the LA ratio is dependent on the base angle with the 90 degree models being more affected by the LA ratio increase. This could mean that there is an angle that maximizes the drop in shear stress as the LA ratio increases. It appears 90 degrees could be one of these angles which could explain why it is the average angle for bifurcations with aneurysms. 
Based on these simulations, it appears aneurysm formation could be linked to both hypothesizes explaining aneurysm genesis. The flow mediated hypothesis is supported by the decrease in max shear stress at areas of high shear stress as the bifurcation angles decrease and LA ratios increase. The minimum shear stress decreases slightly also. These decreases could alter the range of oscillatory shear stress which could stimulant vessel wall remodeling. The fatigue hypothesis is also supported with the expansion of the area affected by high wall shear stress. Healthy vessel walls usually experience shear around 1-7 Pa so the increased area in high shear stress, $16 \mathrm{~Pa}$ or greater, could easily put stress on parts of the vessel that normally don't experience high shear stress. This is further supported by the simulations showing an increase in areas of high shear stress around the MCA bifurcation for geometries that are linked to aneurysm formation. Previous studies also support high wall shear stress as a key factor in stimulating aneurysm genesis. Since aneurysms form at the point of bifurcation, it is likely that the fatigue hypothesis is correct when dealing with aneurysm growth at the MCA bifurcation.

The biggest limitation of the bifurcation models are the ideal geometries. Ideally, digital scans of the main MCA bifurcation would be used from volunteers to get accurate geometries, which would affect the fluid flow and ultimately the mechanical stress on the vessel wall. Modeling the internal carotid artery upstream from the MCA would also get a more accurate inlet flow profile. The inlet flow was perfectly linear, which is not the case seen the PIV experiment from the cadavers which included the ICA. This could alter the outlet flow rate of the daughter arteries and lead to different flow profiles. Another limitation was the SolidWorks models were perfectly smooth tubes with perfect 
curvatures when the artery bifurcated. It is known that human vessels remodel constantly and blemishes appear during maintenance [15]. These flaws can cause micro vortices that could play a role in stimulating aneurysm growth. Another limitation is the mesh size and computation time. Increasing the number of degrees of freedom will always yield more accurate results. 


\section{CHAPTER 6: CONCLUSION}

LA ratios do effect shear stress and fluid flow through the main MCA bifurcation, but only when the MCA bifurcates at angles smaller than homeostasis ranges. Healthy bifurcations with at least one daughter artery bifurcating at 120 degrees show little to no changes in fluid flow or wall shear stress regardless of the angle of the other daughter artery. When the base angle was 90 degrees or less, LA ratios started to cause noticeable drops in the max shear stress over the cardiac cycle, as well as noticeable increases in the area of high shear stress at the bifurcation.

Computer models allow for quick implementation of new features and geometries for future studies. The current models could be used to determine the base angle for which LA ratios start to have noteworthy impact on the magnitude of shear stress and shear area. Additionally, changes to the daughter artery ratios to create asymmetrical artery diameters could be explored in their role in mediating fluid flow and shear stress at the MCA bifurcation. Ideally, MRI scans could be obtained and imported into COMSOL to observe if similar changes in shear stress and fluid flow occur for non-idealized geometries. Finally, aneurysms could be added to the model to explore how geometries affect shear stress and fluid flow in and around aneurysms to better understand why aneurysms grow and rupture.

In conclusion, the models created indicated that geometries related to aneurysms in the MCA cause wall shear stress to decrease and spread out more through the bifurcation. These changes in mechanical stimuli support both leading hypothesizes for aneurysm genesis. Being able to predict conditions that could cause aneurysms can save thousands of lives through pretreatment and altering life styles to limit risk factors. 
Additional information about aneurysms is required to fully understand aneurysm etiology. 


\section{REFERENCES}

1. Takashi Sadatomo, Kiyoshi Yuki, Keisuke Migita, Yasutaka Imada, Masashi Kuwabara, Kaoru Kurisu. "Differences Between Middle Cerebral Artery Bifurcations with Normal Anatomy and those with Aneurysms". Neurosurgery Review 36 (2013): 437-445.

2. Donna L. Hoyert, Jiaquan Xu. "Deaths: Preliminary Data for 2011". Hyattsville, MD : U.S. Department of Health and Human Services (2012).

3. Services, U.S. Department of Health and Human. Cerebral Aneurysms. Bethesda, Maryland : National Institure of Neurological Disorders and Stroke (2013).

4. D. Krex, H. Schackert, G. Schackert. "Genesis of Cerebral Aneurysms - An update". Acta Neurochirurgica 143 (2001): 429-449.

5. Ricardo J. Komotar, J Mocco, Robert A. Solomon. "Guidelines for the Surgical Treatment of Unruptured Intracranial Aneurysms". The 1st Annual J. Lawrence Pool Memorial Research Symposium. New York, NY : s.n. (2008).

6. Jaclyn M. Alfano, John Kolega, Sabaeesh K. Natarajan, Jianping Xiang. "Intracranial Aneurysms Occur More Frequently at Bifurcation Sites That Typically Experience Higher Hemodynamic Stresses". Neurosurgery 73.3 (2013): 497-505.

7. Z. Kulcsar, A. Ugron, M. Narosfoi, Z. Berentei, G. Paal, I. Szikora. "Hemodynamics of Cerebral Anurysm Initiation: The Role of Wall Shear Stress and Spatial Wall Shear Stress Gradient". American Journal of Neuroradiology 32 (2011): 587-594.

8. H.Meng, V Tutino, J Xiang, and A Siddiqui. "High WSS or Low WSS? Complex Interactions of Hemodyanmics with Intracranial Aneurysm Initiation, Growth, and Rupture: Toward a Unifying Hypothesis". American Journal of Neuroradiology 35 (2014): 1254-1262.

9. Masaaki Shojima, Marie Oshima, Kiyoshi Takagi, Ryo Torii. "Magnitude and Role of Wall Shear Stress on Cerebral Aneurysm: Computational Fluid Dynamic Study of 20 Middle Cerebral Artery Aneurysms". Stroke 35 (2004): 2500-2505.

10. T. Tanoue, S. Tateshima, J.P. Villablanca, F. Vinuela, K. Tanishita. "Wall Shear Stress Distribution Inside Growing Cerebral Aneurysm". American Journal of Neuroradiology 32 (2011): 1732-1737.

11. Yoichi Miura, Fujimaro Ishida, Yasuyuki Umeda, Hiroshi Tanemura, Hidenori Suzuki, Satoshi Matsushima, Shinichi Shimosaka, Waro Taki. "Low Wall Shear Stress is Independently Associated with the Rupture Status of Middle Cerebral Artery Aneurysms". Stroke 44 (2013): 519-521. 
12. Luciana Parlea, Rebecca Fahrig, David Holdsworth, and Stephen P. Lownie. "An Analysis of geometry of saccular intracranial aneurysms". American Journal of Nueroradiology 20 (1999) 1079-1089.

13. Michalis Xenos, Yared Alemu, Dan Zamfir, Shmuel Einav, John Ricotta, Nicos Labropoulos, Apostolos Tassiopoulos, Danny Bluestein. "The effect of angulation in Abdominal Aortic Aneurysms: fluid-structure interaction simulations of idealized geometries". Medical and Biological Engineering and Computing 48.12 (2010): 1175-1190.

14. Marieb, Elaine N. Anatomy and Physiology 4th Edition. San Francisco, Ca : Pearson Education, Inc. (2011).

15. Lasheras, Juan C. "The Biomechanics of Arterial Aneurysms". Annual Review of Fluid Mechancis 39 (2007): 293-319.

16. Raymond Vito, Stacey Dixon. "Blood Vessel Constitutive Models: 1995-2002". Annual Review of Biomedical Engineering 5 (2003): 413-439.

17. Andreas Raabe, Jurgen Beck. "Intracranial Aneurysms: New Aspects of Formation, Rupture, and Outcome". Schweizer Archive for Neurologie 162.4 (2011): 169-170.

18. "Cerebral Aneurysm Classification Based on Angioarchitecture". Pritz, Michael B. 2, Journal of Stroke and Cerebrovascualar Diseases 20 (2011): 162-167.

19. Ringer, Andrew. "Endovascular Aneurysm Coiling". Athens, Ohio : Mayfield Clinic \& Spine Institute (2013).

20. Reza Dashti, Juha Hernesniemi, Mika Niemela, Jaakko Rinne, Matti Porras, Martin Lehecka, Hu Shen, Baki Albayrak, Hanna Lehto, Paivi Koroknay-pal, Rafael Sillero de Oliveira, Giancarlo Perra, Antti Ronkainen, Timo Koivisto, Juha Jaaskelainen. "Microneurosurgical management of middle cerebral artery bifurcation aneurysms". Surgical Neurology 67 (2007): 441-456.

21. Seppo Juvela, Kristiina Poussa, Matti Porras. "Factors Affecting Formation and Growth of Intracranial Aneurysms". Stroke 32 (2001): 485-491.

22. Sumera Hasham, Matthew Lewin, Van Tran, Hariyadarshi Pannu, Ann Muilenburg, Marcia Willing, Dianna Milewicz. "Nonsyndromic genetic predisposition to aortic dissection". Annals of Emergency Medicine 43.1 (2004): 79-82.

23. Arthur Ulm, Gregory Fautheree, Necmettin Tanriover, Erminia Albanese, Albert Rhoton, Robert Mericle, Stephen Lewis. "Microsurgical and Angiographic Anatomy of Middle Cerebral Artery Aneurysms: Prevalence and Significance of Early Branch Aneurysms". Operative Neurosurgery 62.2 (2008): 5344-5353.

24. S.B. Pai, R.G. Varma, R.N. Kulkarni. "Microsurgical anatomy of the middle cerebral artery". Neurology India 53.2 (2005): 186-190. 
25. F. Umansky, S. M. Juarez, M. Dujovny, J. I. Ausman, F. G. Diaz, F. Gomes, H. G. Mirchandani, W. J. Ray. "Microsurgical anatomy of the proximal segments of the middle cerebral artery". Journal of Neurosurgery 61.3 (1984): 458-467.

26. Jorge M. Serrador, Paul A. Picot, Brian K. Rutt, J. Kevin Shoemaker, Roberta L. Bondar. "MRI Measures of Middle Cerebral Artery Diameter in Conscious Humans During Simulated Orthostasis". Stroke 31 (2000): 1672-1678.

27. Yoji Nagai, Jeroen Helwegen, Jerome Fleg, Mary Kemper, Christopher Earley, Tomasz Rywik, Pieter Wijn, Jeffrey Metter. "Decay Index: a New Carotid Doppler Waveform Measure Associated with the Windkessel Funtion of Elastic Arteries". Ultrasound in Medicine and Biology 25.9 (1999): 1371-1376.

28. Shigehiko Ogoh, Paul Fadal, Rong Zhang, Christian Selmer, Olvind Jans, Niels Secher, Peter Raven. "Middle Cerebral Artery Flow Velocity and Pulse Pressure During Dynamic Exercise in Humans". American Journal of Physiology - Heart and Circulatory Physiology 288 (2005): H1526-H1531.

29. K W Stock, S G Wetzel, P A Lyrer, E W Radii. "Quantification of Blood Flow in the Middle Cerebral Artery with Phase-Contrast MR Imaging". European Radiology 10 (2000): 1795-1800.

30. Philippe Reymond, Fabrice Merenda, Fabienne Perren, Daniel Rufenacht, Nikos Stergiopulos. "Validation of a one-dimensional model of the systemic arterial tree". American Journal of Physiology - Heart and Circulatory Physiology 297(2009): $\mathrm{H} 208-\mathrm{H} 222$.

31. Jose Manuel Valdueza, Jorn Oliver Balzer, Arno Villringer, Thomas Josef Vogl, Raphael Kutter, Karl Einhaupl. "Changes in Blood Flow Velocity and Diameter of the Middle Cerebral Artery during Hyperventilation: Assessment with MR and Transcranial Doppler Sonography". American Journal of Neuroradiology 18(1997): 1929-1934.

32. Fournier, Ronald L. Basic Transport Phenomena in Biomedical Engineering 3rd Edition. Boca Raton, Florida : CRC press (2012).

33. Nico Westerhof, Jan-Willem Lankhaar. "The Arterial Windkessel". Medical and Biological Engineering and Computing (2008).

34. Marianne Catanho, Mridu Sinha, Varsha Vijayan. Model of Aortic Blood Flow Using the Windkessel Effect. San Diago : University of California of San Diago (2012).

35. Nikos Stergiopulos Berend Westerhof, Nico Westerhof. "Total Arterial Inertance as the Fourth Element of the Windkessel Model". Heart and Circulatory Physiology American Journal of Physiology 276.1 (1999): H81-H88.

36. Howe, Garry. A Multiphysics Simulation of a Coronary Artery. San Luis Obispo : California Polytechnic State University of California (2013). 
37. Mette Olufsen, Ali Nadim, Lewis Lipsitz. "Dynamics of Cerebral Blood Flow Regulation Explained Using a Lumped parameter Model". American Journal of Physiology - Regulatory, Integrative and Comparative Physiology 282 (2002): R611R622.

38. Daniel M. Sforza, Christopher M. Putman, Juan Raul Cebral. "Hemodynamics of Cerebral Aneurysms". Annual Review of Fluid Mechanics 41(2009): 91-107.

39. Anna Marcinkowska-Gapinska, Jacek Gapinski, Waldemar Elikowski, Feliks Jaroszyk. "Comparison of the three rheological models of shear flow behavior studied on blood samples from post infarction patients". Medical and Biological Engineering and computing 45(2007): 837-844.

40. Mark W. Siebert, Petru S.Fodo. "Newtonian and Non-Newtonian Blood Flow over a Backward-facing Step - A Case Study". COMSOL Conderence 2009 Boston. Boston, MA (2009).

41. Theodoros G. Papaioannou, Christodoulos Stefanadis. "Vascular Wall Shear Stree: Basic Principles and Methods". Hellenic Journal of Cardiology 46(2004): 9-15.

42. Berk, Oren Traub and Bradford C. "Laminar Shear Stress: Mechanisms by Which Endothelial Cells Transduce an Atheroprotective Force". Arteriosclerosis, Thrombosis, and Vascular Biology 18 (1998): 677-685.

43. Xixi Zhao, Meide Zhao, Sepideh Amin-Hanjani, Xinjian Du, Sean Ruland, Fady Charbel. "Wall Shear Stress in Major Cerebral Arteries as a Function of Age and Gender - A Study of 301 Healthy Volunteers". American Society of Neuroimaging 00 (2014): 1-5.

44. Haakon M. Lindekleiv, Kristian Valen-Sendstad, Michael K. Morgan, Kent-Andre Mardal. "Sex Differences in Intracranial Arterial Bifurcations". Gender Medicine Vol. 7.2 (2010): 149-155.

45. Wang Sheng-Zhang, Chen Jia-Liang, Ding Guang-hong. "Non-Newtonian Computational Hemodynamics in two Patient-Specific Cerebral Aneurysms with Daughter Saccules". Journal of Hydrodynamics 22.5 (2010): 639-646.

46. Ferguson, Gary. "Physical Factors in the Initiation, Growth, and Rupture of human Intracranial Saccular Aneuryms". Journal of Neurosurgery 37.1 (1972): 666-677.

47. Shegekazu Takeuchi, Takeshi Karino. "Flow Patterns and Distributions of Fluid Velocity and Wall Shear Stress in the Human Internal Carotid and Middle Cerebral Arteries". World Neurosurgery 73.3 (2006): 174-185. 


\section{APPENDIX A: GEOMETRIES FOR SOLIDWORKS MODELS}

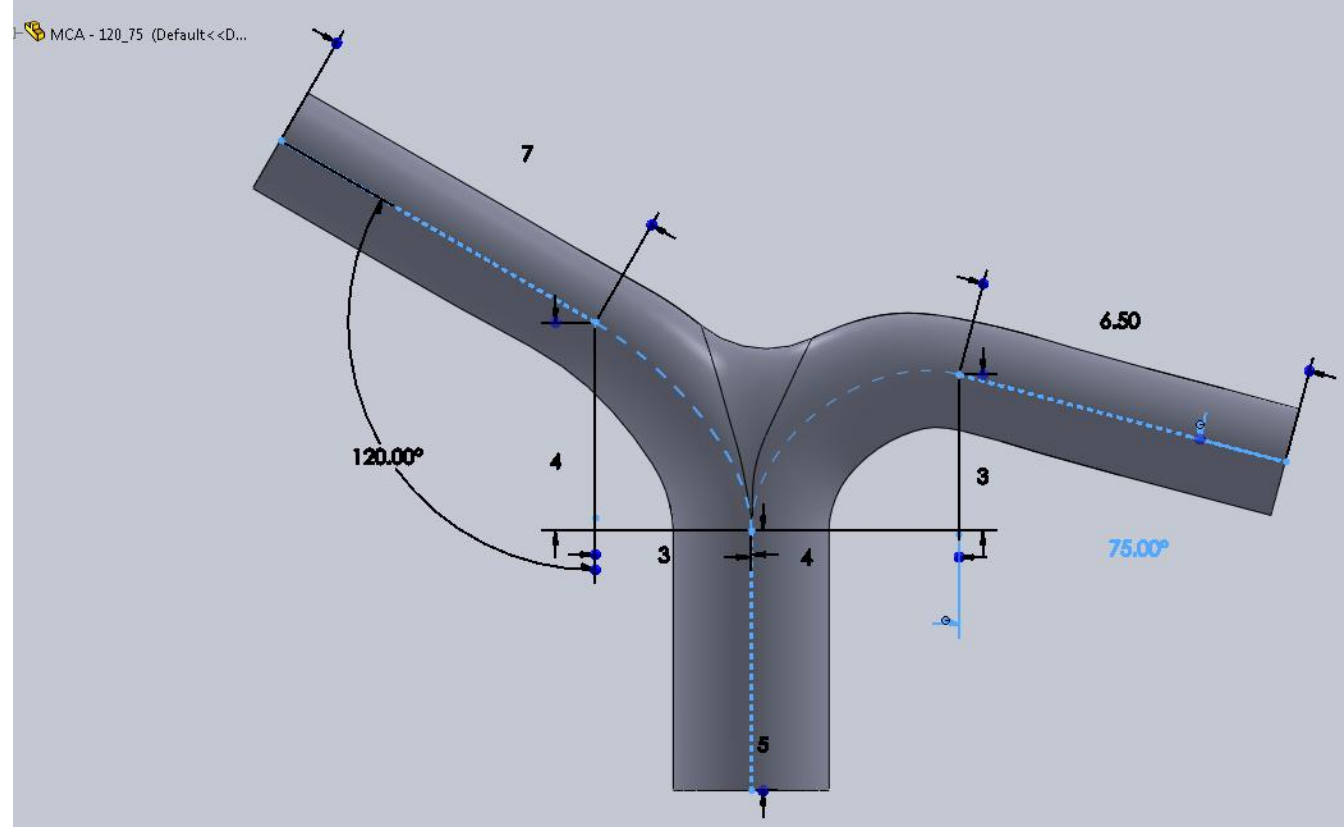

Figure 27: Dimensions of 120 degree base angle model with LA ratio $1.6\left(120^{\circ}-75^{\circ}\right)$.

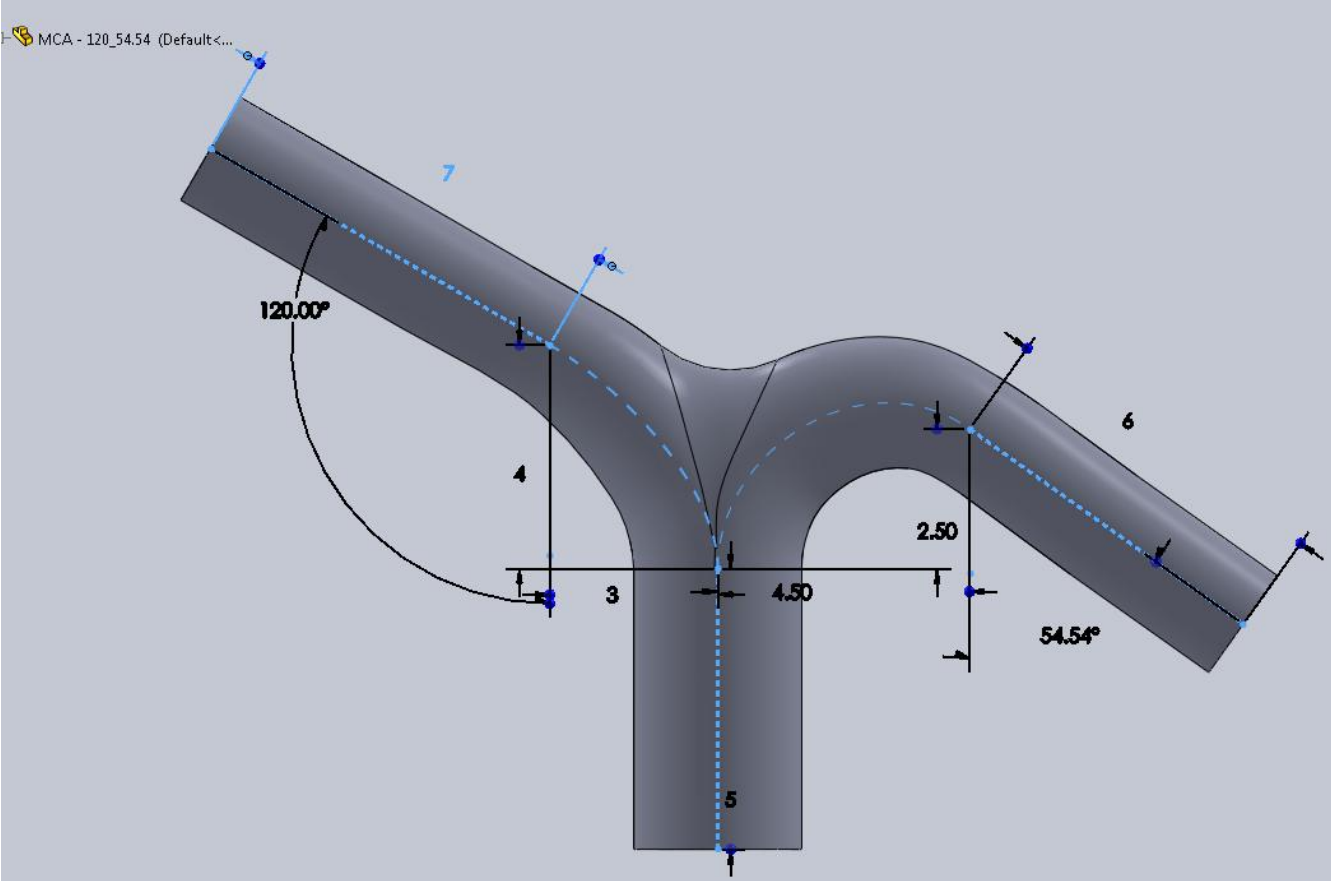

Figure 28: Dimensions of 120 degree base angle model with LA ratio $2.2\left(120^{\circ}-54.54^{\circ}\right)$. 


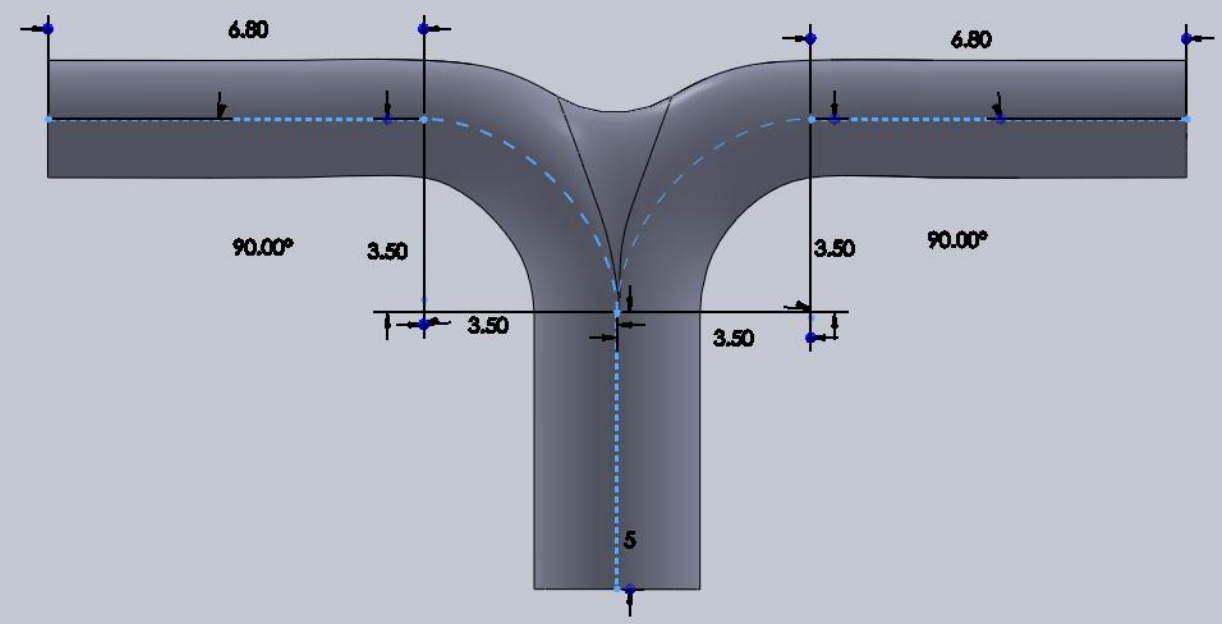

Figure 29: Dimensions of 90 degree base angle model with LA ratio 1 (90 $\left.90^{\circ}-90^{\circ}\right)$

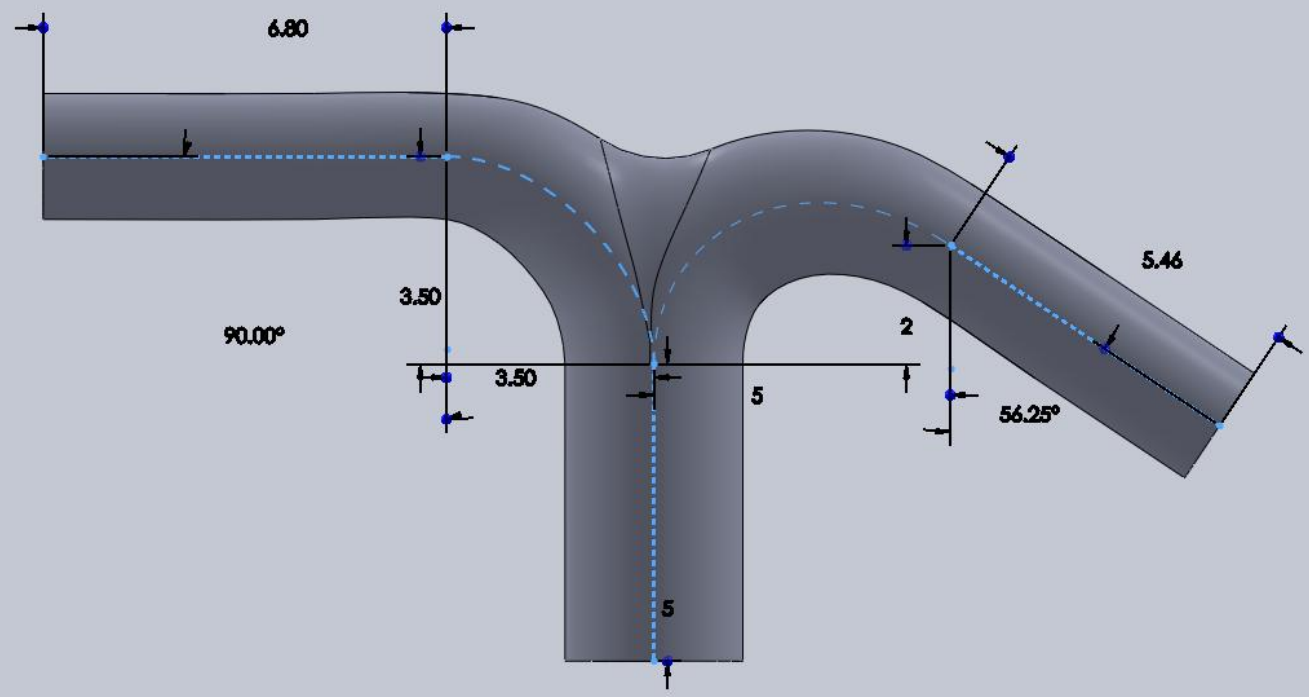

Figure 30: Dimensions of 90 degree base angle model with LA ratio $1.6\left(90^{\circ}-56.25^{\circ}\right)$ 


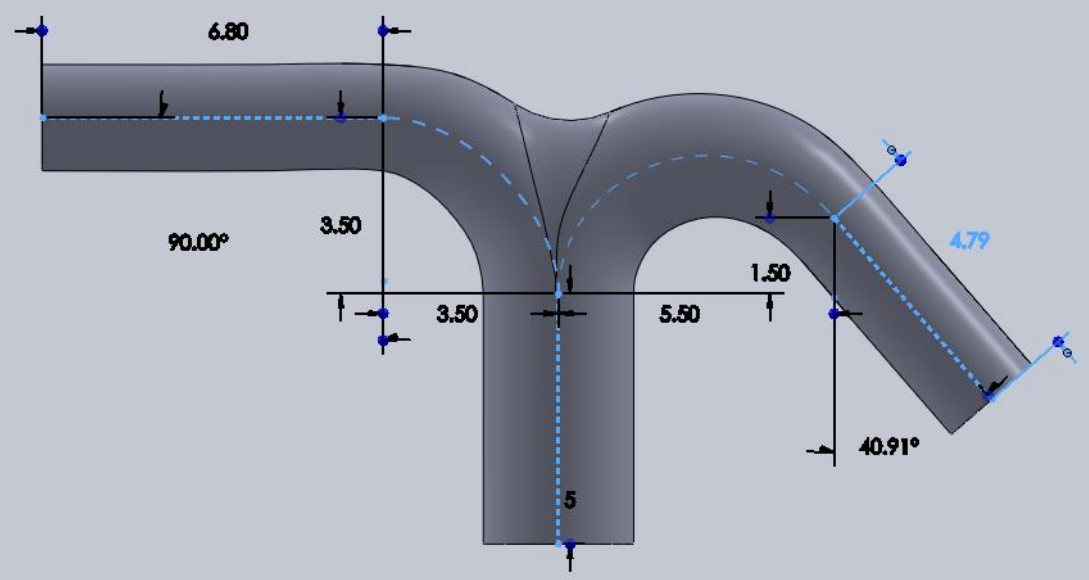

Figure 31: Dimensions of 90 degree base angle model with LA ratio $2.2\left(90^{\circ}-40.91^{\circ}\right)$

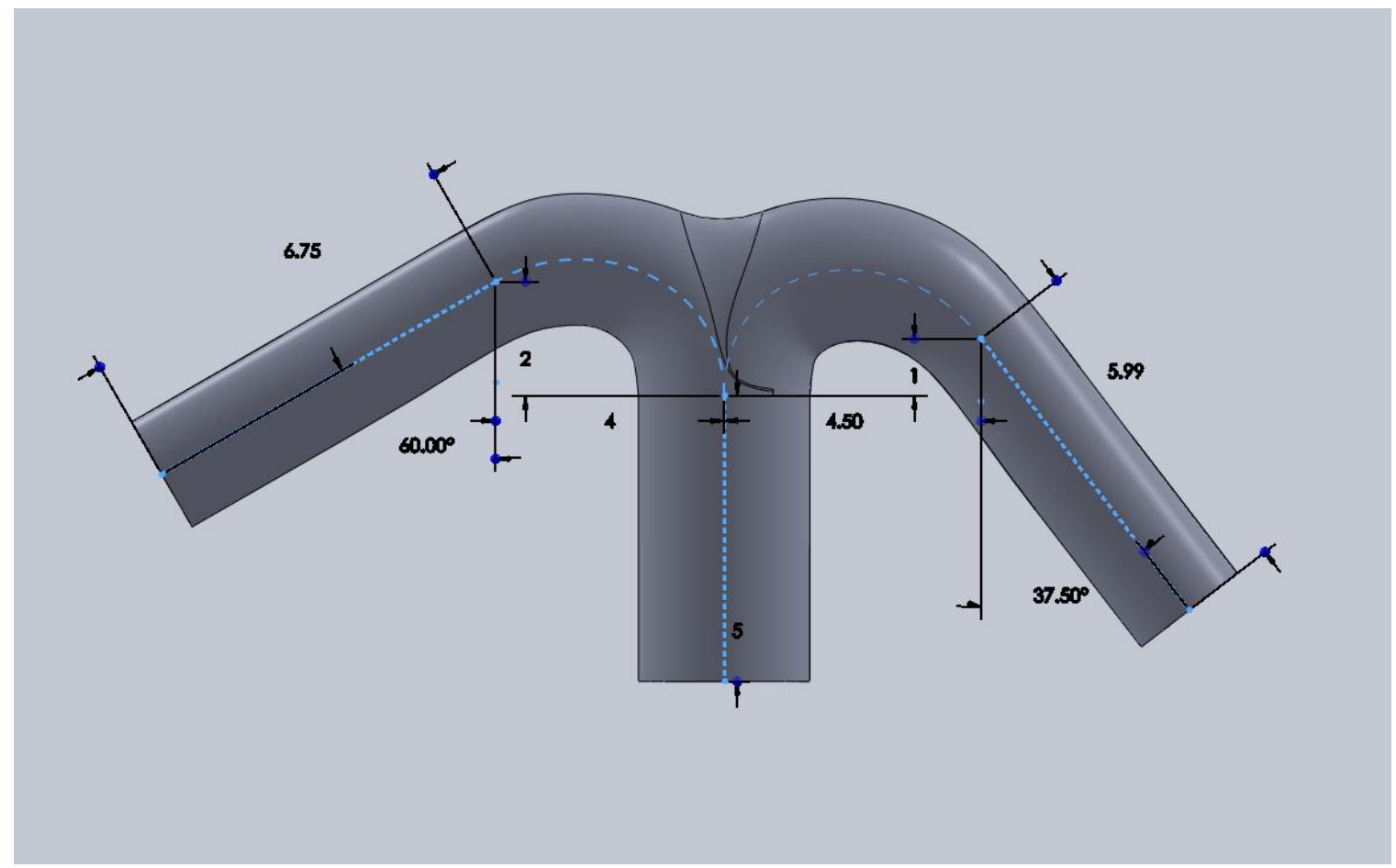

Figure 32: Dimensions of 60 degree base angle model with LA ratio $1.6\left(60^{\circ}-37.5^{\circ}\right)$ 


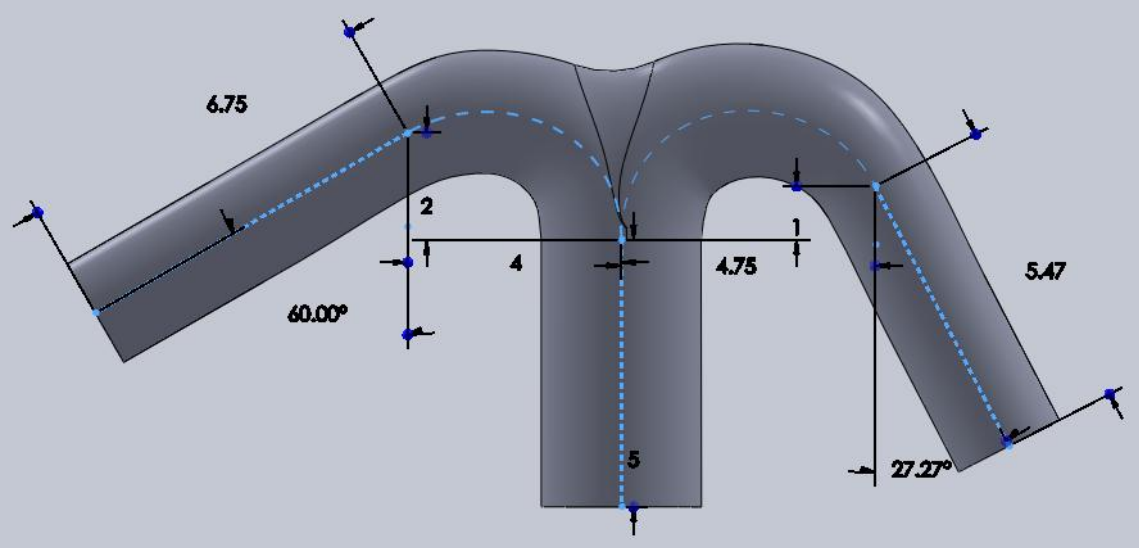

Figure 33: Dimensions of 60 degree base angle model with LA ratio $2.2\left(60^{\circ}-27.27^{\circ}\right)$ 


\section{APPENDIX B: FLOW PROFILE AND SHEAR STRESS PLOTS}

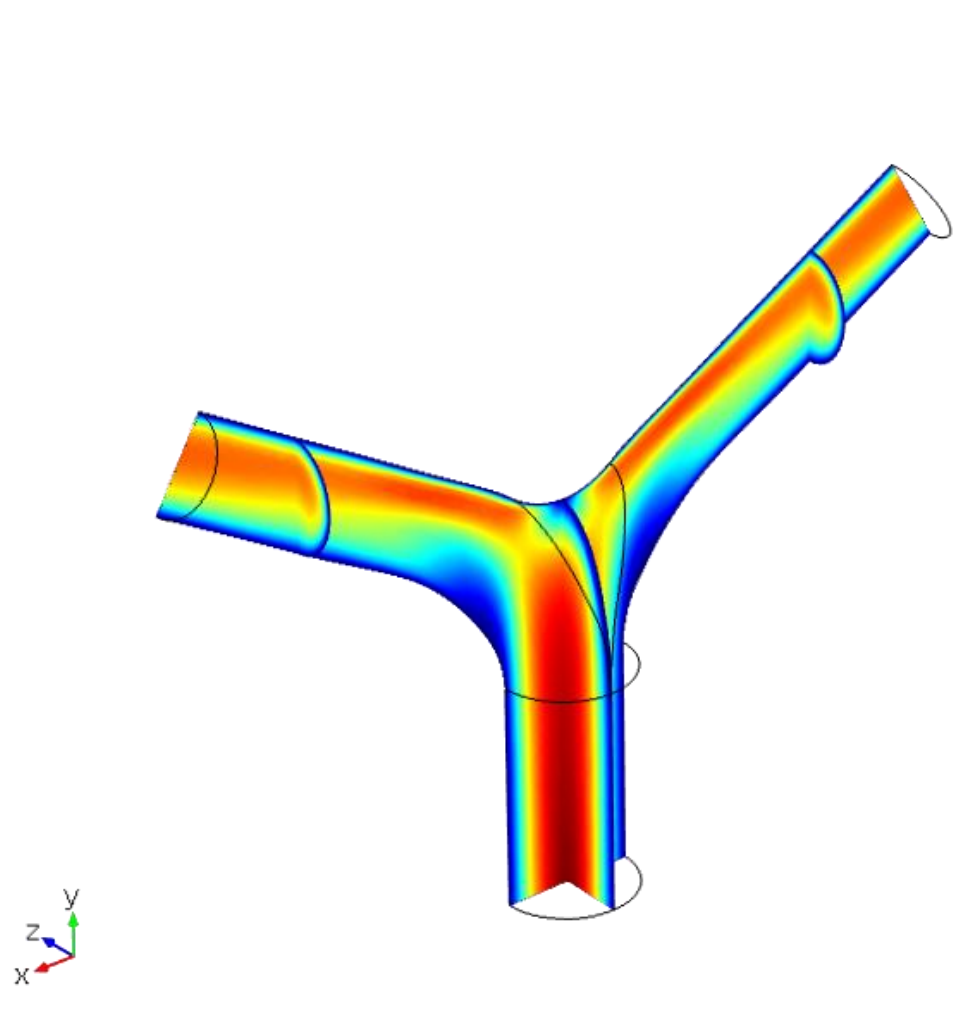

$\Delta 1.02$
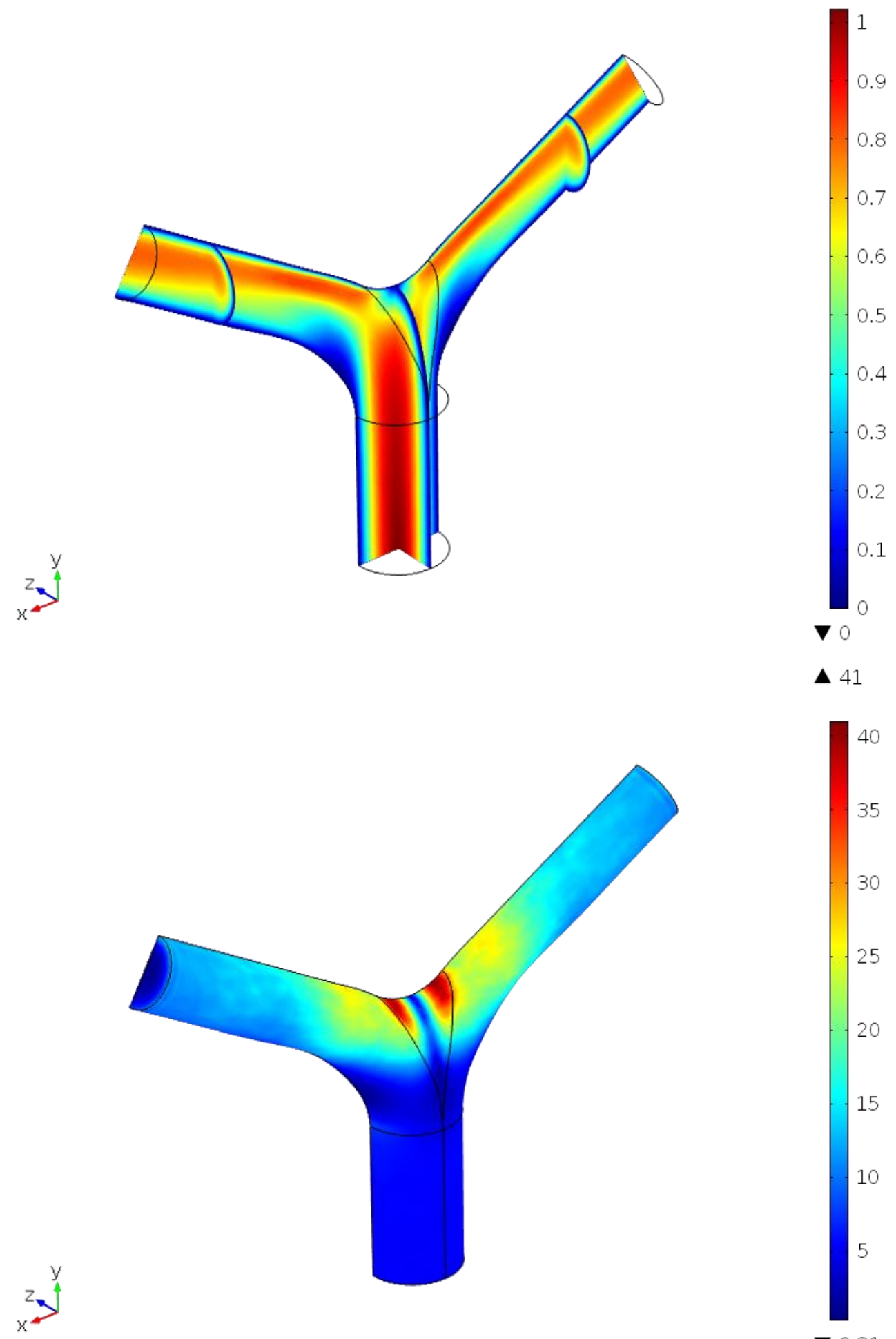

$\Delta 41$

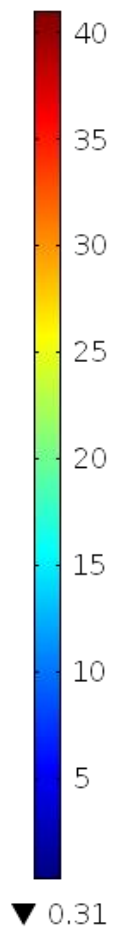

Figure 34: (top)Velocity plot of 120 degree base angle model with LA ratio 1.0.

(bottom)Shear stress plot of 120 degree base angle model with LA ratio 1.0. 


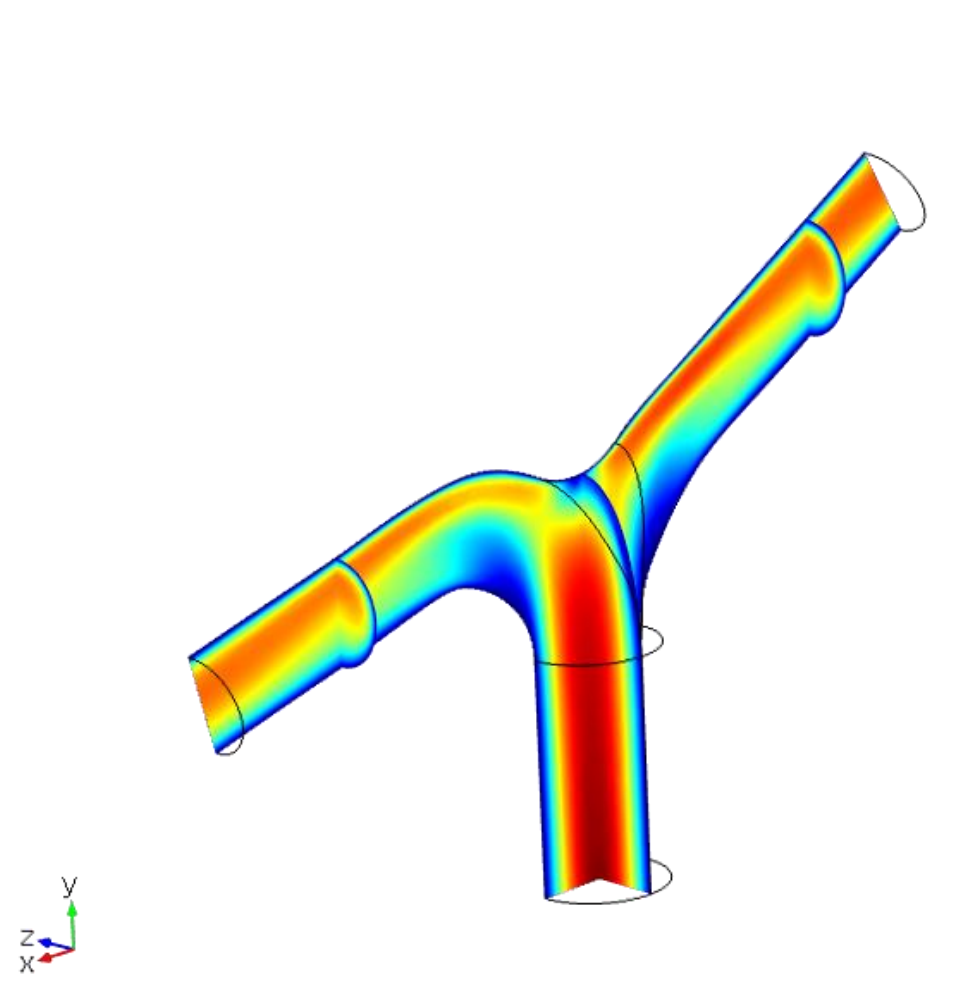

$\Delta 1.01$

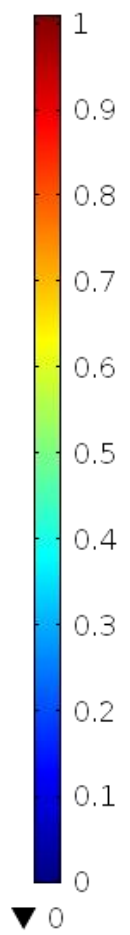

$\Delta 40.4$
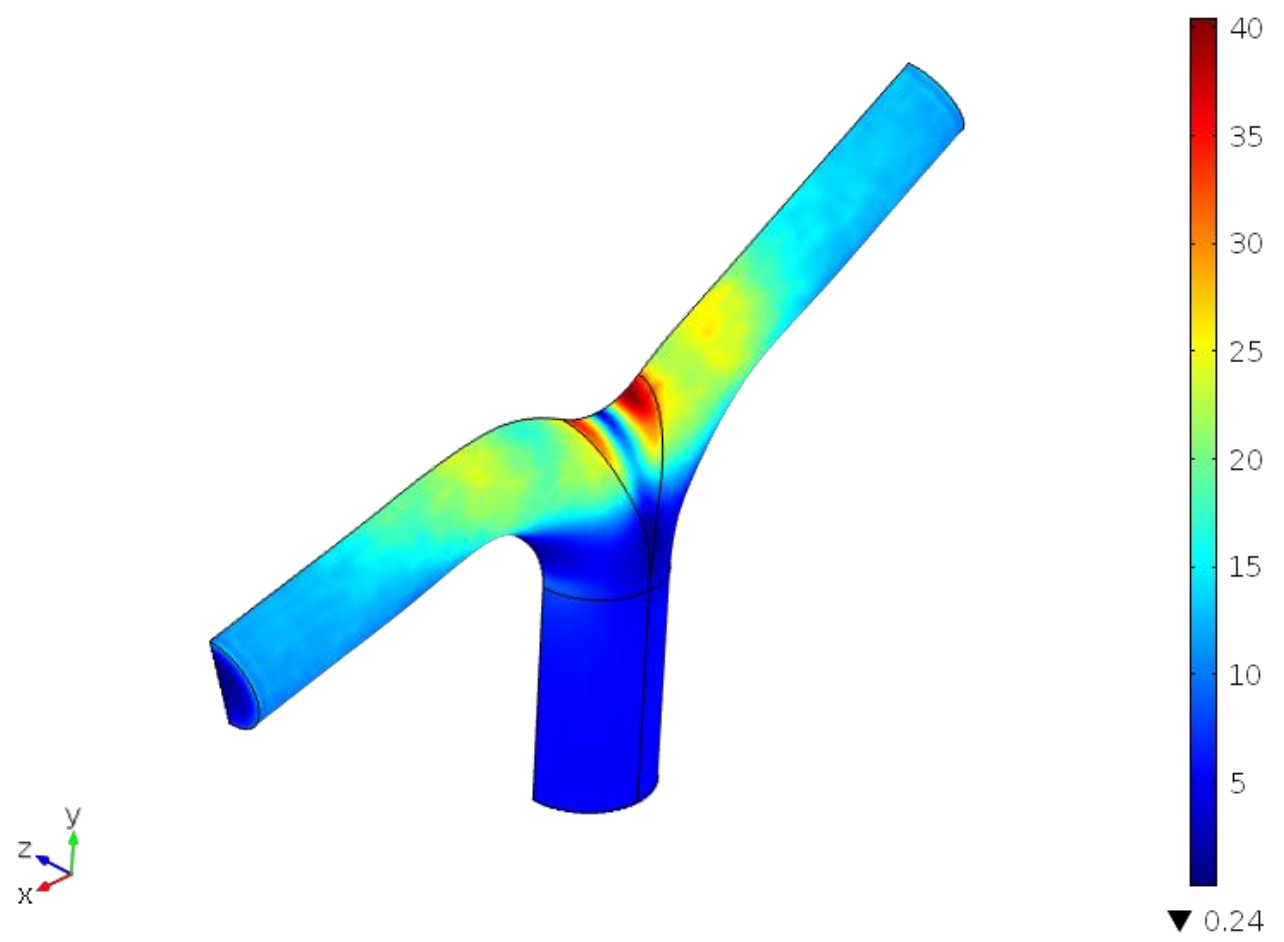

Figure 35: (top)Velocity plot of 120 degree base angle model with LA ratio 1.6.

(bottom)Shear stress plot of 120 degree base angle model with LA ratio 1.6. 
$\boldsymbol{\Delta} 1.01$

$x=$
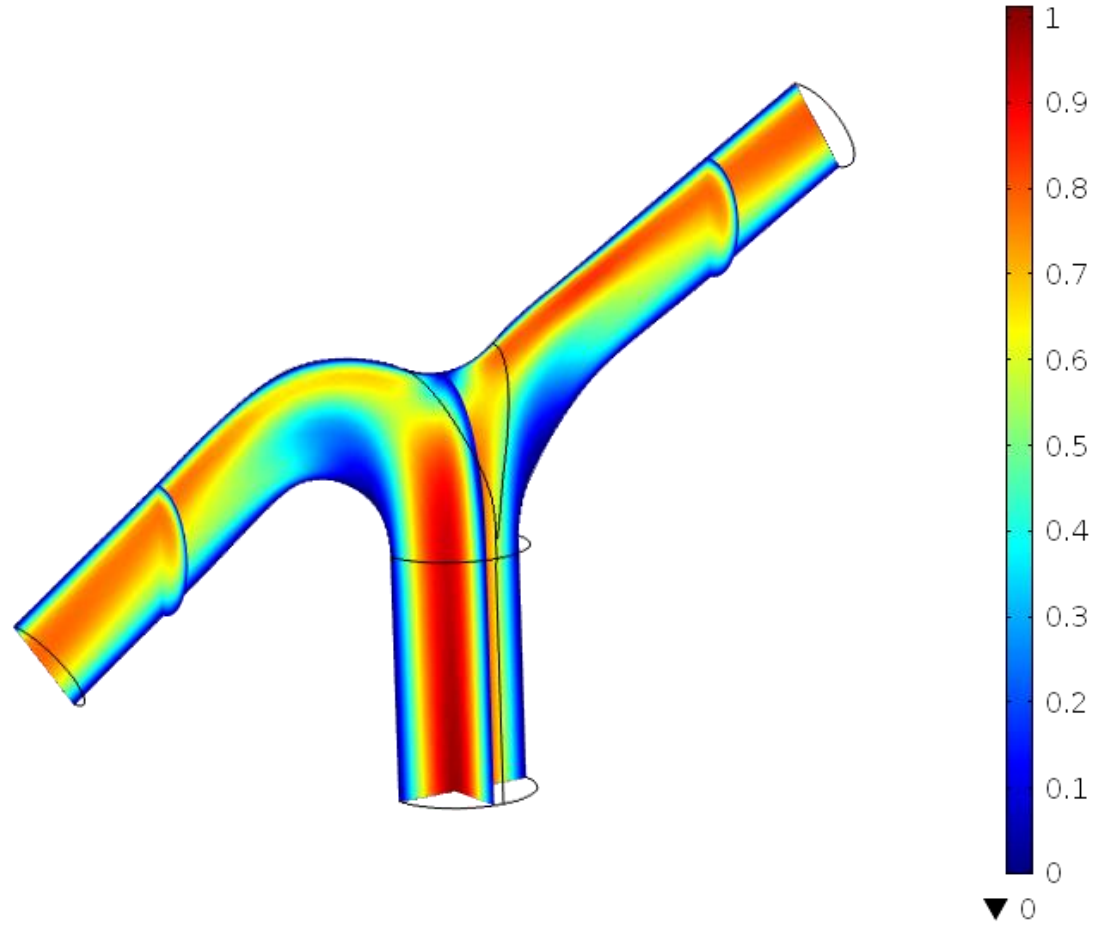

$\Delta 37$
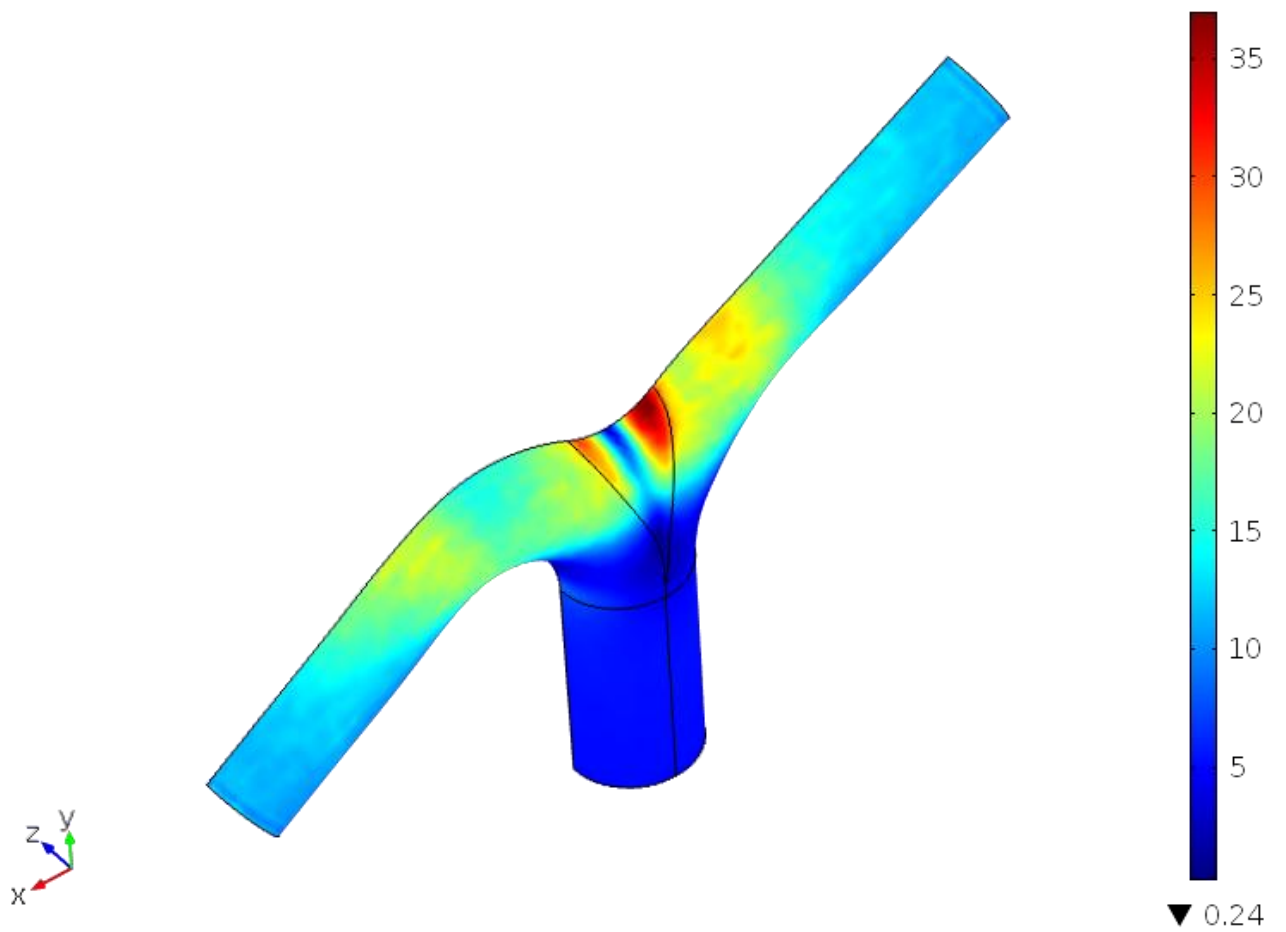

Figure 36: (top)Velocity plot of 120 degree base angle model with LA ratio 2.2.

(bottom)Shear stress plot of 120 degree base angle model with LA ratio 2.2. 


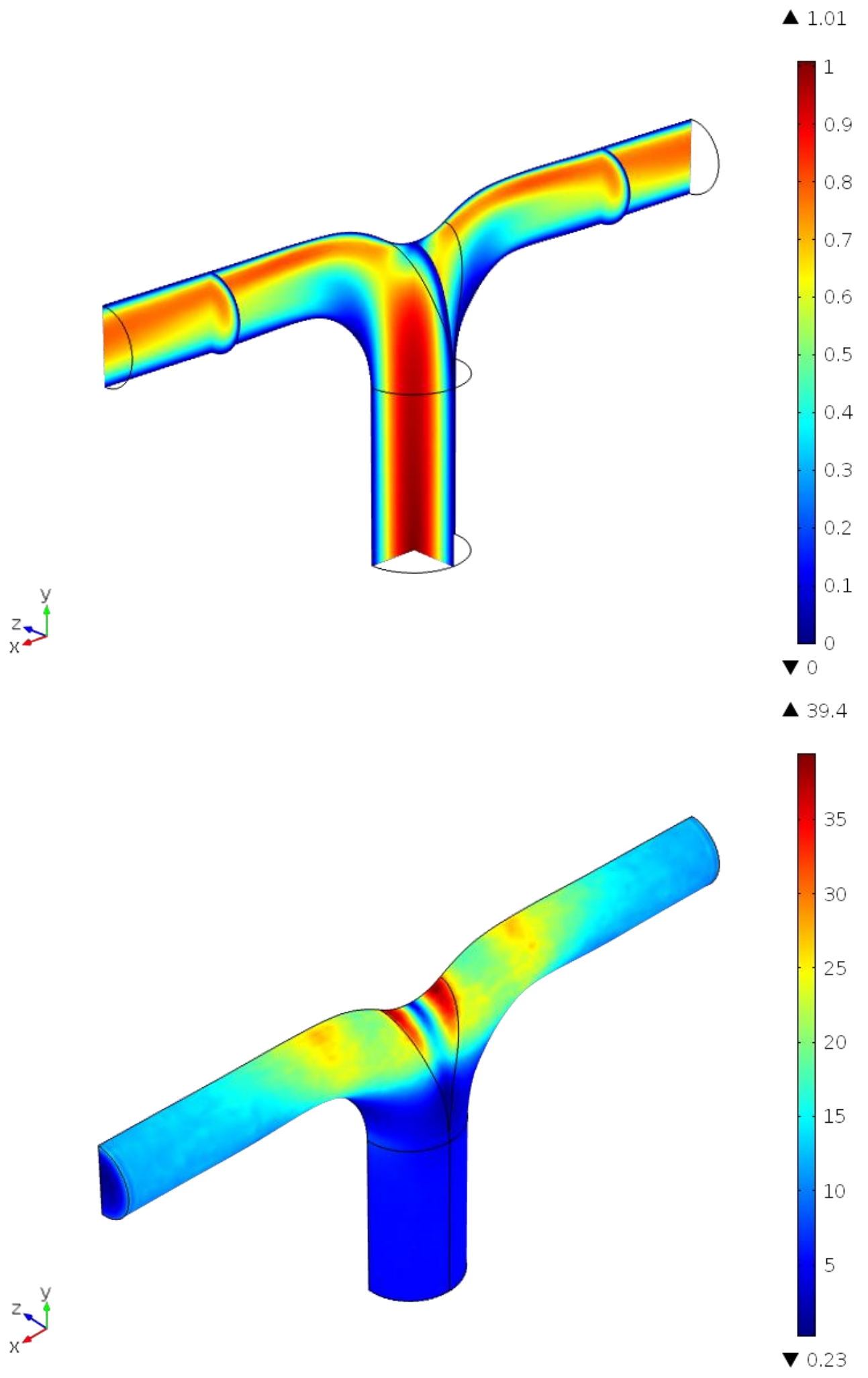

Figure 37: (top)Velocity plot of 90 degree base angle model with LA ratio 1.0. (bottom)Shear stress plot of 90 degree base angle model with LA ratio 1.0. 


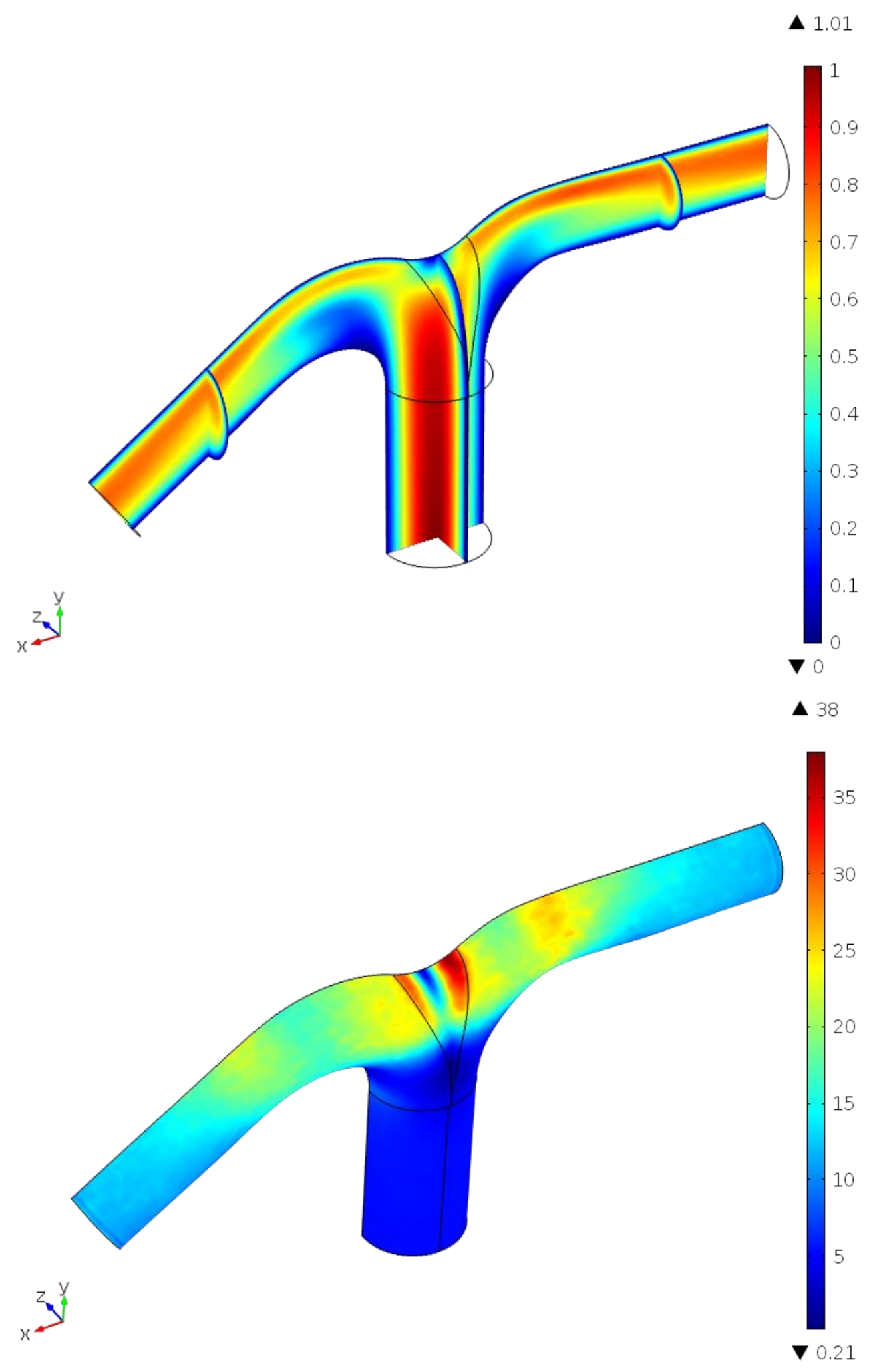

Figure 38: (top)Velocity plot of 90 degree base angle model with LA ratio 1.6.

(bottom)Shear stress plot of 90 degree base angle model with LA ratio 1.6. 

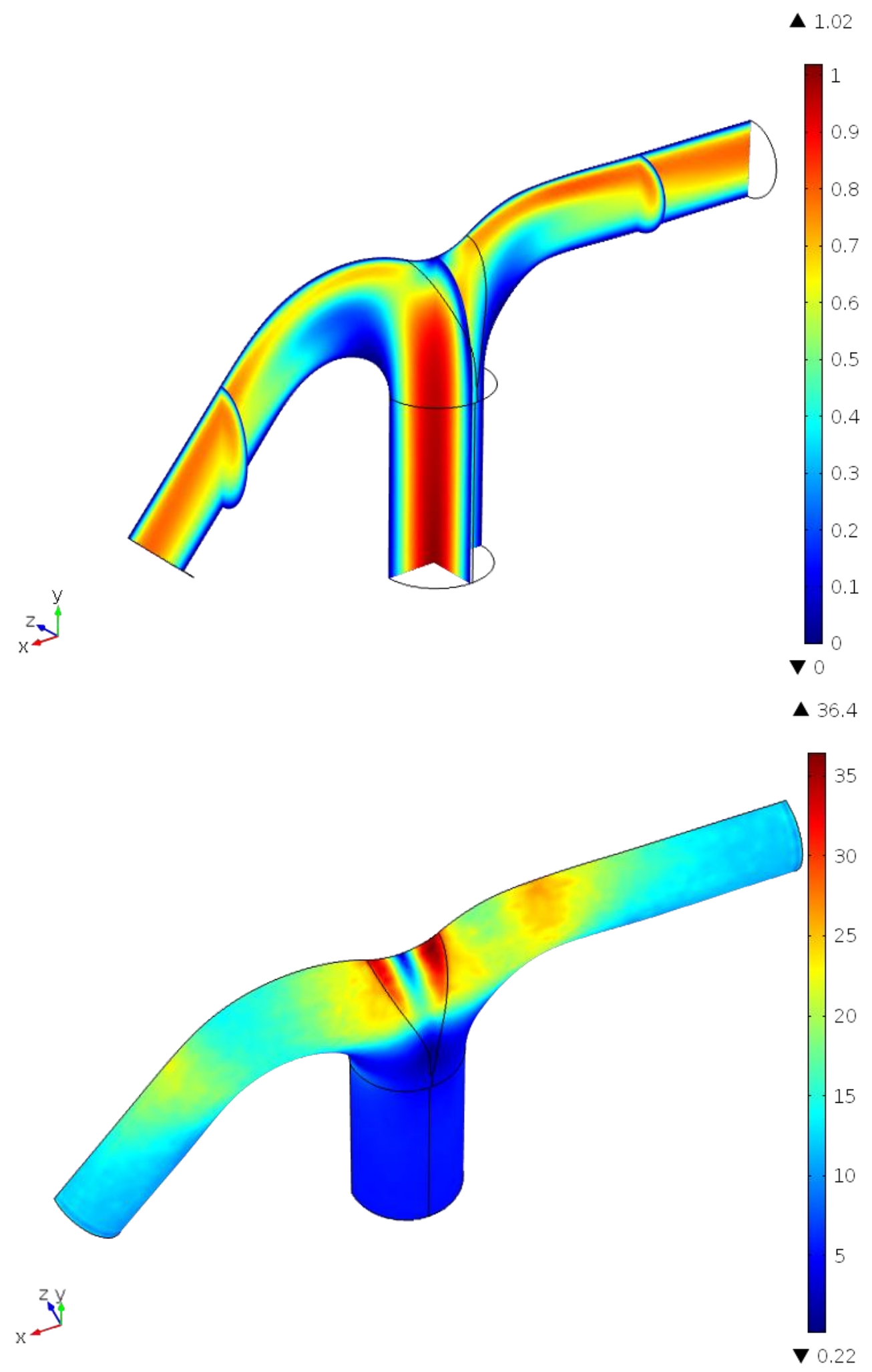

Figure 39: (top)Velocity plot of 90 degree base angle model with LA ratio 2.2.

(bottom)Shear stress plot of 90 degree base angle model with LA ratio 2.2. 


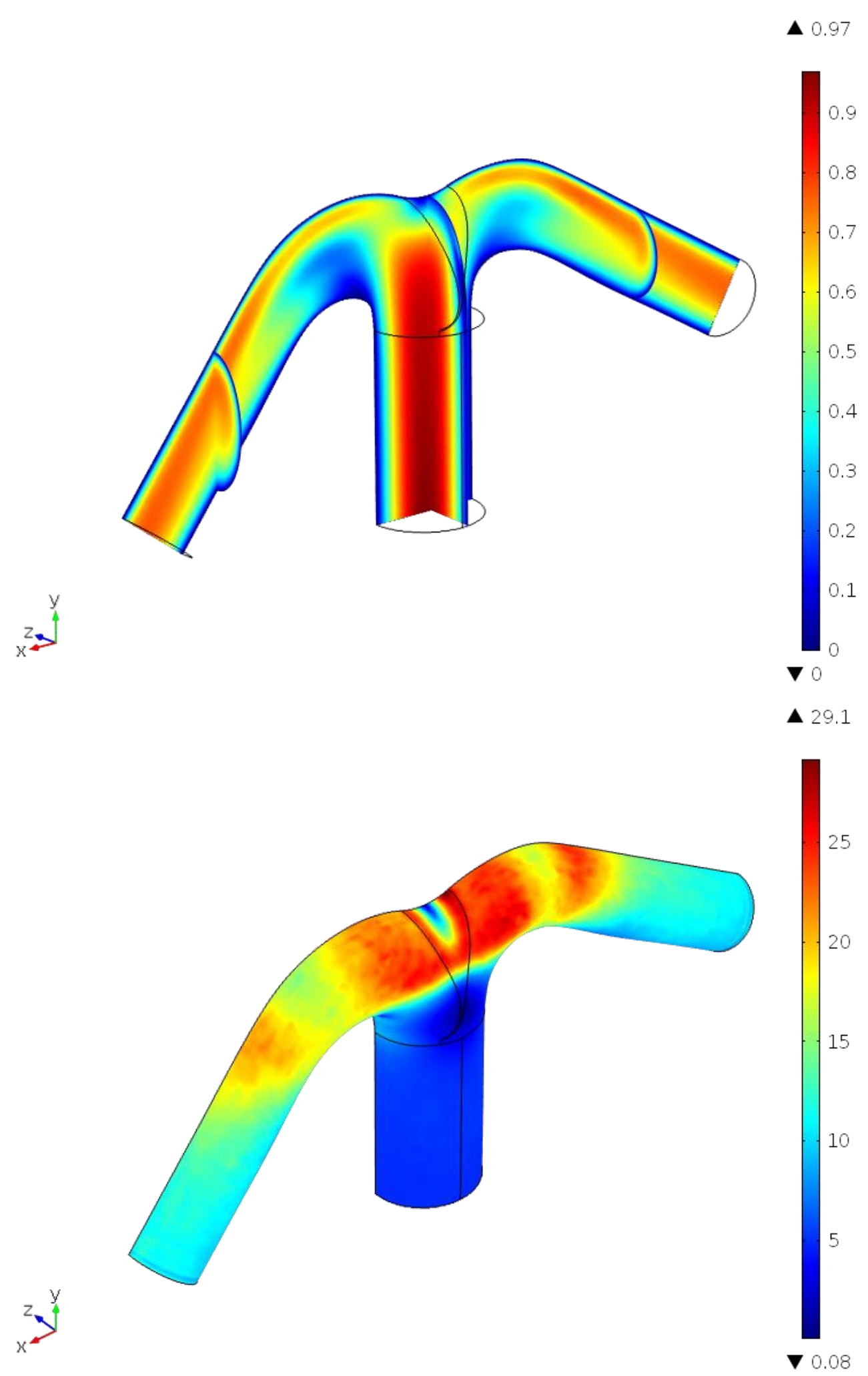

Figure 40: (top)Velocity plot of 60 degree base angle model with LA ratio 1.6.

(bottom)Shear stress plot of 60 degree base angle model with LA ratio 1.6. 


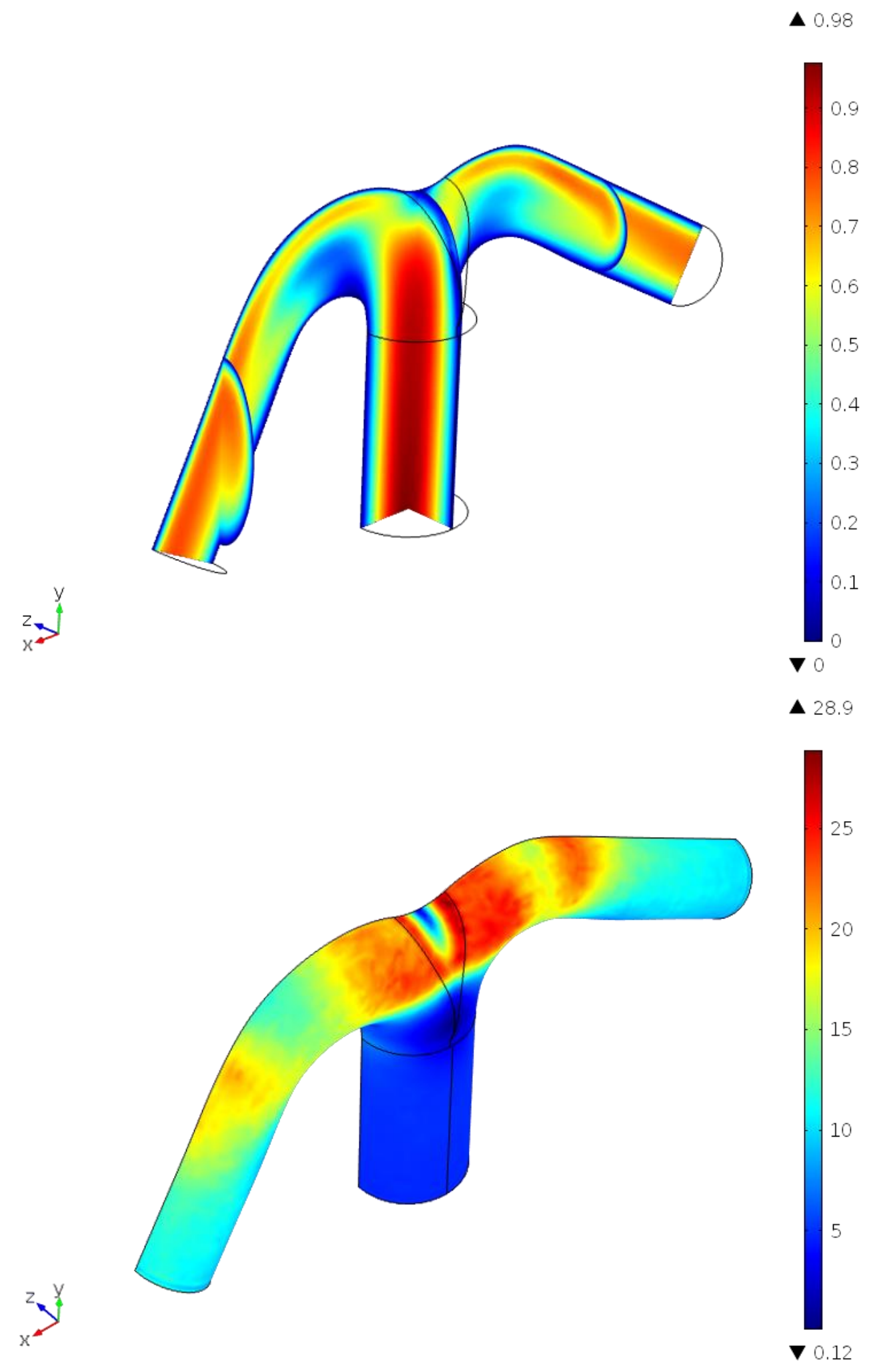

Figure 41: (top)Velocity plot of 60 degree base angle model with LA ratio 2.2.

(bottom)Shear stress plot of 60 degree base angle model with LA ratio 2.2. 


\section{APPENDIX C: MATLAB CODE FOR WINDKESSEL MODEL}

\section{Windkessel.m file code:}

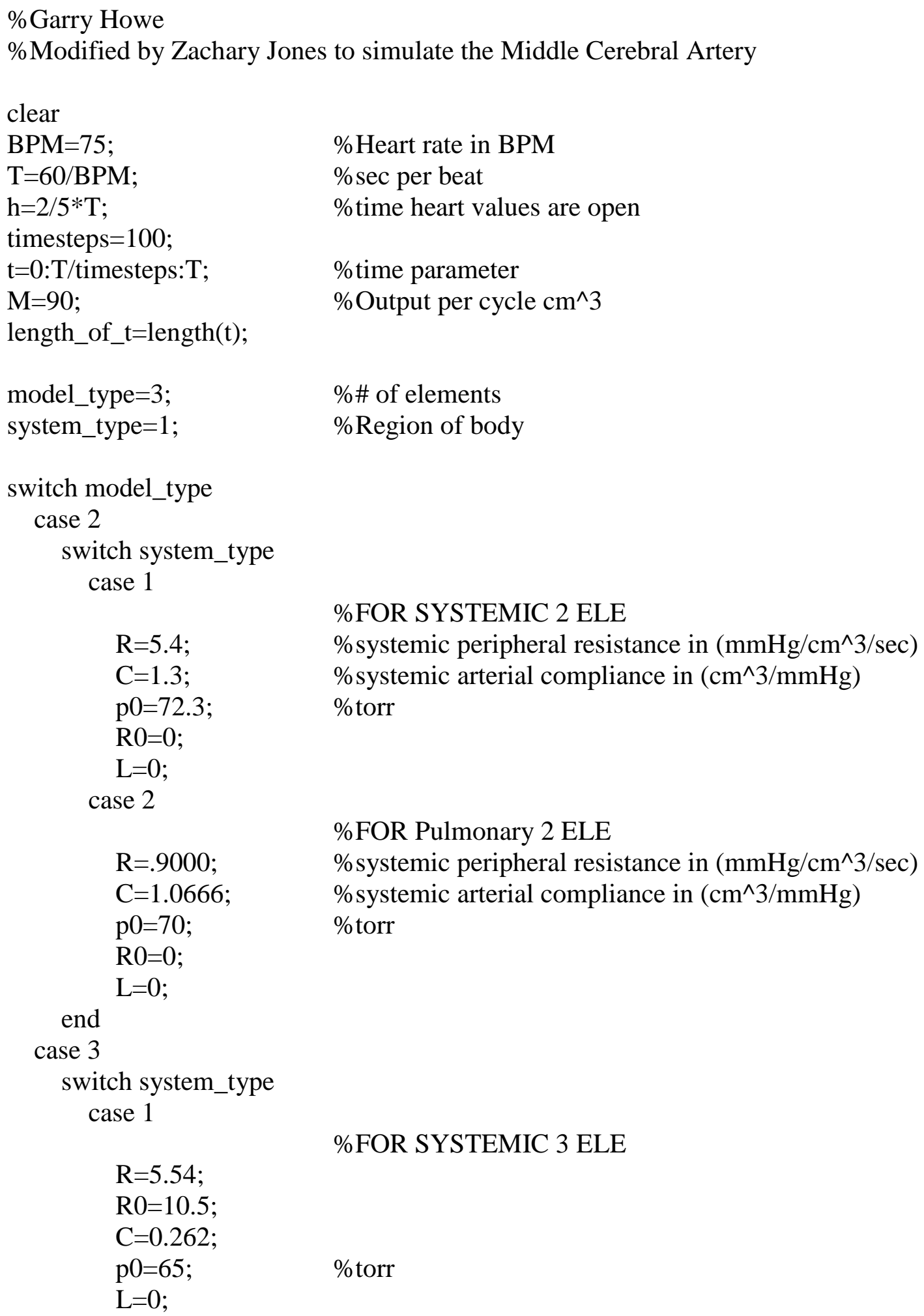




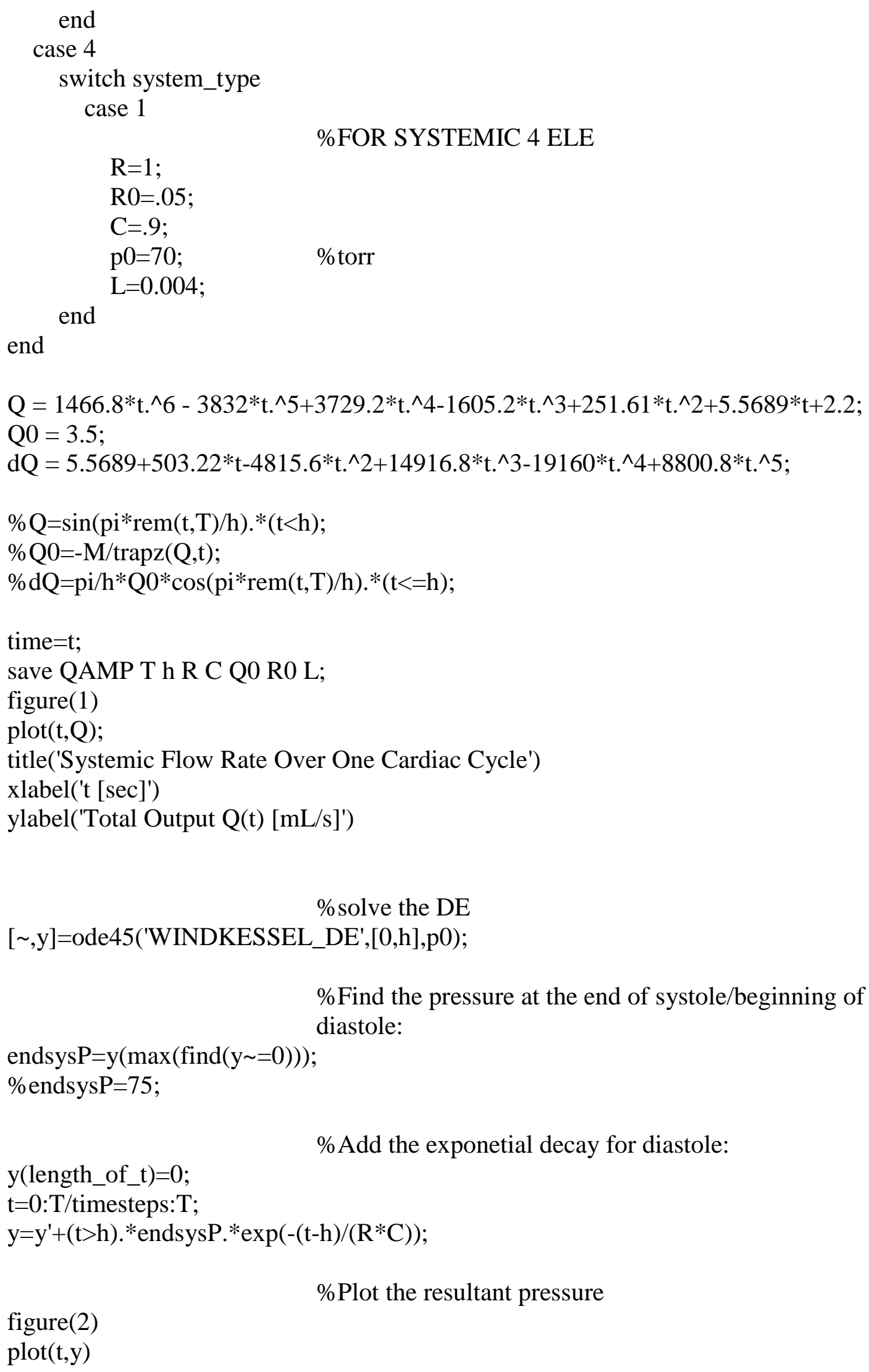


title(['Blood Pressure Over One Cardiac Cycle Predicted by a ',num2str(model_type),' Element Windkessel Model'])

xlabel('t [sec]')

ylabel('Pressure [mmHg]')

\section{Windkessel_DE.m file code:}

function dydt $=$ WINDKESSEL_DE $(t, y)$

load QAMP;

$\mathrm{Q}=1466.8 * \mathrm{t} . \wedge 6-3832 * \mathrm{t} .^{\wedge} 5+3729.2 * \mathrm{t} .^{\wedge} 4-1605.2^{*} \mathrm{t}^{\wedge} 3+251.61 * \mathrm{t} .{ }^{\wedge} 2+5.5689 * \mathrm{t}+2.2$

$\mathrm{dQ}=5.5689+503.22 * \mathrm{t}-4815.6 * \mathrm{t} . \wedge 2+14916.8 * \mathrm{t} .{ }^{\wedge} 3-19160 * \mathrm{t} . \wedge 4+8800.8 * \mathrm{t} . \wedge 5$

$\mathrm{d} 2 \mathrm{Q}=503.22-9631.2 * \mathrm{t}+44750.4 * \mathrm{t}^{\wedge} 2-76640 * \mathrm{t} .{ }^{\wedge} 3+44004 * \mathrm{t} .{ }^{\wedge} 4$;

dydt $=1 / \mathrm{C}^{*}((1+\mathrm{R} 0 / \mathrm{R}) * \mathrm{Q}+(\mathrm{C} * \mathrm{R} 0+\mathrm{L} / \mathrm{R}) * \mathrm{dQ}+\mathrm{L} * \mathrm{C} * \mathrm{~d} 2 \mathrm{Q}-1 / \mathrm{R} * \mathrm{y}) ; \%$ Evalute ODE at time $\mathrm{t}$

end 


\section{APPENDIX D: COMSOL PIV INSTRUCTIONS}

OVERVIEW: This guide will explain how to set up a PIV study for fluid flow modules in COMSOL Multiphysics®. The fluid flow module's study has to be run at least once and can be either a stationary or time dependent study. This guide will be using a stationary study using a laminar flow module.

\section{MODELING INSTRUCTIONS:}

\section{CREATING THE PHYSICS AND STUDY}

1. On the Home toolbar, click on the Add Physics button.

2. In the Add Physics window, navigate and click on Fluid Flow > Particle Tracing for Fluid Flow (ftp).

3. In the Physics in study subsection, find the table below and uncheck Study 1.

\begin{tabular}{l|l}
\hline Study & Solve \\
\hline Study 1 & $\mathrm{x}$ \\
\hline
\end{tabular}

4. In the Add physics window, click Add to Component.

5. On the Home toolbar, click on the Add Study button.

6. In the Add Study window, select Preset Studies> Time Dependent.

7. In the Physics in study subsection, uncheck laminar flow (spf).

\begin{tabular}{l|l}
\hline Physics & Solve \\
\hline Laminar Flow (spf) & $\mathrm{x}$ \\
\hline
\end{tabular}

8. In the Add Study window, click Add Study.

These step creates a new particle tracing for fluid flow (FTP) that uses a new time dependent study. This is done so that the FTP study is independent from the study used to simulate the fluid flow allowing changes to one without affecting the other.

\section{SETTINGS FOR PARTICLE TRACING FOR FLUID FLOW}

9. In the model builder tree, right click on Particle tracing for fluid flow (ftp) and click on Drag Force.

10. Select the domain being simulate. 
11. In the Drag force setting windows under the Drag Force section, find the $\mathbf{u}$ and from the list, choose Velocity field (spf/fp 1).

12. In the same section, find $\boldsymbol{\mu}$ and from the list, choose Dynamic Viscosity (spf/fp 1).

This adds a drag force to each particle that uses the pre simulated velocity and viscosity to determine the force applied to each particle.

13. In the Model builder tree, right click on Particle tracing for fluid flow (ftp) and click on Inlet.

14. Select the Boundaries you wish to act as an inlet for the particles

15. In the Inlet settings window, find the Initial Position section.

16. From the Initial Position list, choose Density.

17. Set the $\mathbf{N}$ field to the number of particles to be released

18. Set the $\boldsymbol{\rho}$ field to $\mathbf{1}$ ( 1 indicates a uniform distribution).

19. Find the Initial Velocity section and from the $\mathbf{u}$ list, pick Velocity field (spf/fp 1).

Multiple boundaries can be selected as inlets and the same boundary can have multiple different inlets. $\rho$ can be equations or expressions leading to distributions based on model geometries or flow velocities.

20. In the Model builder tree, right click on Particle tracing for fluid flow (ftp) and click on Outlet.

21. Select the desired outlets.

22. In the Outlet settings window, set Wall condition to Disappear.

23. In the Model builder tree, navigate to Particle Tracing for Fluid Flow > Particle Properties 1.

24. In the Particle Properties settings, find $\boldsymbol{\rho}_{\mathbf{p}}$ field and set the density for the particles.

25. In the Particle Properties settings, find $\mathbf{d}_{\mathbf{p}}$ field and set the diameter for the particles.

26. Leave Charge Number at 0 . 
27. In the Model builder tree, navigate to the study you created in the beginning and click on Step 1: Time Dependent.

28. In the Time Dependent settings window, find the Values of dependent variable section.

29. Select the Values of variables not solved for check box.

30. From the Method list, choose Solution.

31. From the Study list, choose Study 1, Stationary (or the study used to solve the fluid flow).

The last 3 steps cause missing variables for the particle tracing for fluid flow to be taken from study that solved for the fluid flow. This step is important or else the particles will not move.

32. Find the Study Setting section and locate the Range button.

33. In the Range dialog box, set the Step to a desired time step

34. In the Stop field, type in the length of the particle simulation.

35. Click Replace

36. on the Home toolbar, click Compute. 


\section{APPENDIX E: LIVELINK ${ }^{\mathrm{tm}}$ AND SOLIDWORKS®}

\section{SETTING UP LIVELINK:}

Setting up a Livelink ${ }^{\mathrm{tm}}$ to SolidWorks ${ }^{\circledR}$ is very quick. The two requirements is to have both SolidWorks with the file to be livelinked and the COMSOL model that is to receive the Livelink open at the same time. If both are not open, the Livelink will fail when synchronized.

1. Open COMSOL Multiphysics ${ }^{\circledR}$

2. Open or create a new 3D model

3. Open or create the SolidWorks ${ }^{\circledR}$ that is to be Livelinked

4. In the Model Builder, right click geometries and click on Livelink for SolidWorks.

5. In the Livelink for SolidWorks setting window, find the Import Options.

6. Change the Length unit option to From CAD document.

7. Click Synchronize.

Note: Any changes made to the model in SolidWorks requires the Livelink to be resynchronized by pressing the Synchronize button.

Additionally, Livelink can pass variables between COMSOL and SolidWorks. This can be used to set up parametric sweeps inside COMSOL using models from SolidWorks.

1. In the Livelink for SolidWorks setting window, find the Parameters in CAD Packages. A table below should appear in the settings window.

\begin{tabular}{l|l}
\hline Name & Value \\
\hline & \\
\hline
\end{tabular}

2. In SolidWorks, find the name of the dimension you wish to Livelink to COMSOL.

3. Put the name of the dimension into the table above.

4. Set the value to a number, variable, or predefined parameter.

5. Any changes made in COMSOL to the value of the Livelinked variable will change the SolidWorks model when Synchronize or when a study is computed. If a parametric sweep is performed, the SolidWorks model will update with each step of the sweep. 